JOURNAL OF THE

AMERICAN MATHEMATICAL SOCIETY

Volume 14, Number 4, Pages 783-841

S 0894-0347(01)00371-X

Article electronically published on May 30, 2001

\title{
COMPLEX BRJUNO FUNCTIONS
}

\author{
STEFANO MARMI, PIERRE MOUSSA, AND JEAN-CHRISTOPHE YOCCOZ
}

This paper is dedicated to Michael R. Herman

\section{INTRODUCTION}

1.1. The real Brjuno function. Let $\alpha \in \mathbb{R} \backslash \mathbb{Q}$ and let $\left(p_{n} / q_{n}\right)_{n>0}$ be the sequence of the convergents of its continued fraction expansion. A Brjuno number is an irrational number $\alpha$ such that $\sum_{n=0}^{\infty} \frac{\log q_{n+1}}{q_{n}}<+\infty$.

The importance of Brjuno numbers comes from the study of one-dimensional analytic small divisors problems. In the case of germs of holomorphic diffeomorphisms of one complex variable with an indifferent fixed point, extending a previous result of Siegel [S], Brjuno proved ([Br1], $[\mathrm{Br} 2])$ that all germs with linear part $\lambda=e^{2 \pi i \alpha}$ are linearizable if $\alpha$ is a Brjuno number. Conversely the third author proved that this condition is also necessary [Yo1]. Similar results hold for the local conjugacy of analytic diffeomorphisms of the circle ([KH], [Yo2], [Yo3] $)$ and for some area-preserving maps ([Ma], Da1] $)$, including the standard family (Da2], BG1, [BG2]). The set of Brjuno numbers is invariant under the action of the modular group PGL $(2, \mathbb{Z})$ and it can be characterized as the set where the Brjuno function $B: \mathbb{R} \backslash \mathbb{Q} \rightarrow \mathbb{R} \cup\{+\infty\}$ is finite.

This arithmetical function is $\mathbb{Z}$-periodic and satisfies the remarkable functional equation

$$
B(\alpha)=-\log \alpha+\alpha B\left(\frac{1}{\alpha}\right), \quad \alpha \in(0,1),
$$

which allows $B$ to be interpreted as a cocycle under the action of the modular group (see Appendix 5 for details). In terms of the continued fraction expansion of $\alpha$ the Brjuno function is defined as follows:

$$
B(\alpha)=\sum_{j=0}^{+\infty} \beta_{j-1}(\alpha) \log \alpha_{j}^{-1},
$$

where $\beta_{-1}=1, \beta_{j}(\alpha)=\left|p_{j}-q_{j} \alpha\right|(j \geq 0), \alpha_{j}=-\frac{q_{j} \alpha-p_{j}}{q_{j-1} \alpha-p_{j-1}}$ (see Appendix 1 for a short summary of the relevant facts concerning the continued fraction).

Received by the editors February 16, 2000.

2000 Mathematics Subject Classification. Primary 37F50, 11A55, 32A40; Secondary 37F25, 46F15, 20G99.

Key words and phrases. Small divisors, continued fractions, Bruno functions, complex boundary behaviour, renormalisation, hyperfunctions, modular group, dilogarithm.

This work began during a visit of the first author at the S.Ph.T.-CEA/Saclay and at the Department of Mathematics of Orsay during the academic year 1993-1994. This research has been supported by the CNR, CNRS, INFN, MURST and an EEC grant. 
In a previous paper [MMY] we introduced the linear operator

$$
T f(x)=x f\left(\frac{1}{x}\right), \quad x \in(0,1),
$$

acting in the space of $\mathbb{Z}$-periodic measurable functions and we studied the equation

$$
(1-T) B_{f}=f,
$$

so that

$$
\begin{aligned}
& B_{f}(x+1)=B_{f}(x) \quad \forall x \in \mathbb{R}, \\
& B_{f}(x)=f(x)+x B_{f}(1 / x) \quad \forall x \in(0,1) .
\end{aligned}
$$

The choice $f(x)=-\log \{x\}$ (where $\{\cdot\}$ denotes the fractional part) leads to the Brjuno function $B$. For other choices of the singular behaviour of $f$ at 0 the condition $B_{f}<+\infty$ leads to different diophantine conditions. On the other hand if $f$ is Hölder continuous, then $B_{f}$ is also Hölder continuous and this fact could help to explain the numerical results of Buric, Percival and Vivaldi BPV].

Acting on $L^{p}([0,1])$ the operator $T$ has spectral radius bounded above by $\frac{\sqrt{5}-1}{2}$ (thus $(1-T)$ is invertible). A suitable adaptation of this argument has led us to conclude that the Brjuno function belongs to $\mathrm{BMO}\left(\mathbb{T}^{1}\right)$ (bounded mean oscillation; see references [Ga], GCRF for its definition and more information).

By Fefferman's duality theorem, BMO is the dual of the Hardy space $H^{1}$; thus one can add an $L^{\infty}$ function to $B$ so that the harmonic conjugate of the sum will also be $L^{\infty}$. This suggests we look for a holomorphic function $\mathcal{B}$ defined on the upper half plane which is $\mathbb{Z}$-periodic and whose trace on $\mathbb{R}$ has for imaginary part the Brjuno function $B$. The function $\mathcal{B}$ will be called the complex Brjuno function.

Another motivation for the introduction of the complex Brjuno function comes from results concerning the problem of the linearization of the quadratic polynomial $P_{\lambda}(z)=\lambda\left(z-z^{2}\right)$ ([Yo1], Chapter II). One has the following results:

(1) there exists a bounded holomorphic function $U: \mathbb{D} \rightarrow \mathbb{C}$ such that $|U(\lambda)|$ is equal to the radius of convergence of the normalized linearization of $P_{\lambda}$;

(2) for all $\lambda_{0} \in \mathbb{S}^{1},|U(\lambda)|$ has a non-tangential limit in $\lambda_{0}$ (which is still equal to the radius of convergence of the normalized linearization of $P_{\lambda_{0}}$ );

(3) if $\lambda=e^{2 \pi i \alpha}, \alpha \in \mathbb{R} \backslash \mathbb{Q}, P_{\lambda}$ is linearizable if and only if $\alpha$ is a Brjuno number. Moreover there exists a universal constant $C_{1}>0$ and for all $\varepsilon>0$ there exists $C_{\varepsilon}>0$ such that for all Brjuno numbers $\alpha$ one has

$$
(1-\varepsilon) B(\alpha)-C_{\varepsilon} \leq-\log |U(\lambda)| \leq B(\alpha)+C_{1} .
$$

In [MMY] the authors proposed the following conjecture (see also [Ma]): the function defined on the set of Brjuno numbers by $\alpha \mapsto B(\alpha)+\log \left|U\left(e^{2 \pi i \alpha}\right)\right|$ extends to a $1 / 2-$ Hölder continuous function as $\alpha$ varies in $\mathbb{R}$. If this were true, then the function $-i \mathcal{B}(z)+\log U\left(e^{2 \pi i z}\right)$ would also extend to a Hölder continuous function on $\overline{\mathbb{H}}$.

1.2. The complex Brjuno function. A natural question now is how to extend the operator $T$ to complex analytic functions. This is achieved as follows: the operator $T$ extends to the space $A^{\prime}([0,1])$ of hyperfunctions $u$ with support contained in $[0,1]$ (see Section 1.4 for a proof of this fact and Appendix 2 for a very brief introduction to hyperfunctions). This space is canonically isomorphic to the complex vector space $\mathcal{O}^{1}(\overline{\mathbb{C}} \backslash[0,1])$ of holomorphic functions on $\overline{\mathbb{C}} \backslash[0,1]$ which vanish at infinity. The connection between $u$ and the associated holomorphic function $\varphi$ 
is commonly written as: $u(x)=\frac{1}{2 i}(\varphi(x+i 0)-\varphi(x-i 0))$, which is also equal to $\mathfrak{I} m \varphi(x+i 0)$ when $\varphi$ is real (i.e. $\varphi(\bar{z})=\overline{\varphi(z)})$. On $\mathcal{O}^{1}(\overline{\mathbb{C}} \backslash[0,1])$ the formula for $T$ reads

$$
(T \varphi)(z)=-z \sum_{m=1}^{\infty}\left[\varphi\left(\frac{1}{z}-m\right)-\varphi(-m)\right]+\sum_{m=1}^{\infty} \varphi^{\prime}(-m) .
$$

Formally we have

$$
(1-T)^{-1} \varphi(z)=\sum_{r \geq 0}\left(T^{r} \varphi\right)(z)=\sum_{g \in \mathcal{M}}\left(L_{g} \varphi\right)(z),
$$

where the monoid

$$
\mathcal{M}=\left\{g=\left(\begin{array}{ll}
a & b \\
c & d
\end{array}\right) \in \operatorname{GL}(2, \mathbb{Z}), d \geq b \geq a \geq 0, \text { and } d \geq c \geq a\right\} \cup\left\{\left(\begin{array}{ll}
1 & 0 \\
0 & 1
\end{array}\right)\right\}
$$

acts on $\mathcal{O}^{1}(\overline{\mathbb{C}} \backslash[0,1])$ according to

$$
\left(L_{g} \varphi\right)(z)=(a-c z)\left[\varphi\left(\frac{d z-b}{a-c z}\right)-\varphi\left(-\frac{d}{c}\right)\right]-\operatorname{det}(g) c^{-1} \varphi^{\prime}\left(-\frac{d}{c}\right) .
$$

The series (1.4) actually converges in $\mathcal{O}^{1}(\overline{\mathbb{C}} \backslash[0,1])$ to a function $\sum_{\mathcal{M}} \varphi$. To recover a holomorphic periodic function on $\mathbb{H}$ one sums over integer translates:

$$
\mathcal{B}_{\varphi}(z)=\sum_{n \in \mathbb{Z}}\left(\sum_{\mathcal{M}} \varphi\right)(z-n) .
$$

To construct the complex Brjuno function one has to take $\varphi_{0}(z)=-\frac{1}{\pi} \operatorname{Li}_{2}\left(\frac{1}{z}\right)$, where $\mathrm{Li}_{2}$ is the dilogarithm (Appendix 3, [0]). Then the above formulas give

$$
\begin{aligned}
\mathcal{B}(z) & =-\frac{1}{\pi} \sum_{p / q \in \mathbb{Q}}\left\{\left(p^{\prime}-q^{\prime} z\right)\left[\operatorname{Li}_{2}\left(\frac{p^{\prime}-q^{\prime} z}{q z-p}\right)-\operatorname{Li}_{2}\left(-\frac{q^{\prime}}{q}\right)\right]\right. \\
& \left.+\left(p^{\prime \prime}-q^{\prime \prime} z\right)\left[\operatorname{Li}_{2}\left(\frac{p^{\prime \prime}-q^{\prime \prime} z}{q z-p}\right)-\operatorname{Li}_{2}\left(-\frac{q^{\prime \prime}}{q}\right)\right]+\frac{1}{q} \log \frac{q+q^{\prime \prime}}{q+q^{\prime}}\right\},
\end{aligned}
$$

where $\left[\frac{p^{\prime}}{q^{\prime}}, \frac{p^{\prime \prime}}{q^{\prime \prime}}\right]$ is the Farey interval such that $\frac{p}{q}=\frac{p^{\prime}+p^{\prime \prime}}{q^{\prime}+q^{\prime \prime}}$ (with the convention $p^{\prime}=p-1, q^{\prime}=1, p^{\prime \prime}=1, q^{\prime \prime}=0$ if $\left.q=1\right)$.

1.3. Main results: Properties of the complex Brjuno function. Our main result (Corollary 5.8) is that the real part of $\mathcal{B}$ is bounded on the upper half plane (note that this statement is stronger than the result obtained by the above mentioned general properties of the harmonic conjugates of BMO functions); moreover the trace of $\mathfrak{R} \mathcal{B}$ on $\mathbb{R}$ is continuous at all irrational points and has a jump of $\pi / q$ at each rational point $p / q \in \mathbb{Q}$ (see Section 5.2.9).

A numerical study of the function $\arg U\left(e^{2 \pi i \alpha}\right)$ seems to indicate a similar behaviour (see Figure 1).

Concerning the boundary behaviour of the imaginary part of $\mathcal{B}$ we prove the following (Theorem 5.19):

(i) if $\alpha$ is a Brjuno number, then $\Im m \mathcal{B}(\alpha+w)$ converges to $B(\alpha)$ as $w \rightarrow 0$ in any domain with a finite order of tangency to the real axis;

(ii) if $\alpha$ is diophantine, one can allow domains with infinite order of tangency (see $(5.58))$. 


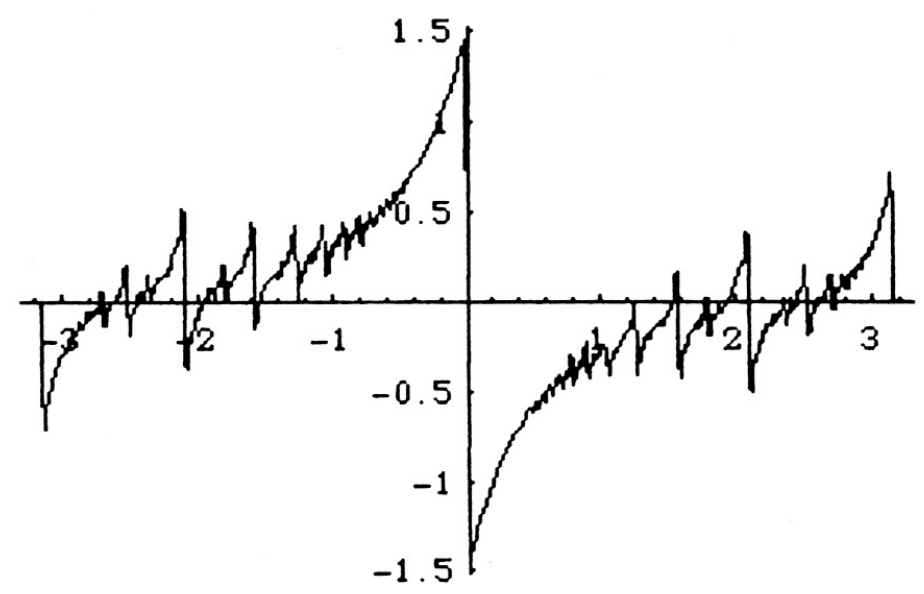

Figure 1. The argument of the function $U$ close to the unit circle: plot of $\arg U\left(0.999 e^{i x}\right)$ for $-\pi<x<\pi$.

The precise behaviour of $\Im m \mathcal{B}$ at rational points is described by Theorem 5.10.

1.4. Hyperfunctions and operator $T$. Let $u, \psi \in L^{2}([0,1]), m \in \mathbb{N}, m \geq 1$. We consider

$$
T_{m} u(x)=\left\{\begin{array}{cc}
x u(1 / x-m) & \text { if } x \in\left[\frac{1}{m+1}, \frac{1}{m}\right], \\
0 & \text { otherwise. }
\end{array}\right.
$$

Note that $T_{m} u=\left.(T u)\right|_{\left[\frac{1}{m+1}, \frac{1}{m}\right]}$, thus $T=\sum_{m \geq 1} T_{m}$. We define the adjoint $T_{m}^{*}$ by

$$
\int_{0}^{1} T_{m} u(x) \psi(x) d x=\int_{0}^{1} u(x) T_{m}^{*} \psi(x) d x
$$

which gives

$$
T_{m}^{*} \psi(x)=\frac{1}{(m+x)^{3}} \psi\left(\frac{1}{m+x}\right) .
$$

The previous formula with $\psi$ analytic in a neighborhood of $[0,1]$ allows us to extend the domain of definition of $T_{m}$ to the hyperfunctions $u \in A^{\prime}([0,1])$ (see Appendix 5 for a very short summary of hyperfunctions) and to obtain $T_{m} u \in A^{\prime}\left(\left[\frac{1}{m+1}, \frac{1}{m}\right]\right)$. More generally, if $u \in A^{\prime}\left(\left[\gamma_{0}, \gamma_{1}\right]\right), \gamma_{0}>-1$, then $T_{m} u \in A^{\prime}\left(\left[\frac{1}{m+\gamma_{1}}, \frac{1}{m+\gamma_{0}}\right]\right) \subset$ $A^{\prime}\left(\left[0, \frac{1}{1+\gamma_{0}}\right]\right)$. One has

$$
\int_{0}^{1} T u(x) \psi(x) d x=\sum_{m \geq 1} \int_{0}^{1} u(x) T_{m}^{*} \psi(x) d x .
$$

If $\psi$ is holomorphic in a neighborhood $V$ of $[0,1]$, then also $T_{m}^{*} \psi$ is holomorphic in $V$ and one has

$$
\sup _{V}\left|T_{m}^{*} \psi\right| \leq \frac{1}{2} m^{-3} \sup _{V}|\psi|
$$

(this follows immediately from the estimates of Section 3.2 choosing $V$ to be the complement of a neighborhood of $D_{\infty}$ with respect to the Poincaré metric on $\overline{\mathbb{C}} \backslash$ 
$[0,1])$. Therefore the series $\sum_{m \geq 1} T_{m} u$ converges in $A^{\prime}([0,1])$ to a hyperfunction which will be denoted $T u$.

Let $u \in A^{\prime}\left(\left[\gamma_{0}, \gamma_{1}\right]\right), \gamma_{0}>-1$, and let $\varphi \in \mathcal{O}^{1}\left(\overline{\mathbb{C}} \backslash\left[\gamma_{0}, \gamma_{1}\right]\right)$ be the associated holomorphic function, i.e. $\varphi$ is holomorphic outside $\left[\gamma_{0}, \gamma_{1}\right]$ and vanishes at infinity. For all $m \geq 1$ the holomorphic function associated to $T_{m} u$ is $L_{g(m)} \varphi$ given by equation (1.5) with $a=0, b=c=1, d=m$. Indeed if $z \notin\left[\gamma_{0}, \gamma_{1}\right]$

$$
\left(T_{m} u\right)\left(c_{z}\right)=u\left(T_{m}^{*} c_{z}\right)
$$

where $c_{z}(x)=\frac{1}{\pi} \frac{1}{x-z}$, and

$$
\begin{aligned}
\pi T_{m}^{*} c_{z}(x) & =\frac{1}{(m+x)^{3}} \frac{1}{\frac{1}{m+x}-z}=\frac{1}{(m+x)^{2}}-z\left(\frac{1}{m+x-\frac{1}{z}}-\frac{1}{m+x}\right) \\
& =\pi\left[\left.\frac{\partial}{\partial z} c_{z}(x)\right|_{z=-m}-z\left(c_{\frac{1}{z}-m}(x)-c_{-m}(x)\right)\right] .
\end{aligned}
$$

Thus we are led to define $T \varphi$, according to (1.3), as an element of the space $\mathcal{O}^{1}\left(\overline{\mathbb{C}} \backslash\left[0,1 /\left(1+\gamma_{0}\right)\right]\right)$.

To construct the complex analytic extension of the functions $B_{f}$ (defined in Section 1.1) our strategy is the following:

1) take the restriction of the periodic function $f$ to the interval $[0,1]$;

2) consider its associated hyperfunction $u_{f}$ and its holomorphic representative $\varphi \in \mathcal{O}^{1}(\overline{\mathbb{C}} \backslash[0,1])$.

Then the series (1.6) converges (thanks to Corollary 3.6) to the complex extension $\mathcal{B}_{f}$ of the function $B_{f}$. The main difficulty (unless $f$ belongs to some $L^{p}$ space; see Section 4.3) would be to recover $B_{f}$ as non-tangential limit of the imaginary part of $\mathcal{B}_{f}$ as $\mathfrak{I} m z \rightarrow 0$.

1.5. Summary of the contents. Let us now briefly describe the contents of this article.

In Section 2 we discuss the relation between the monoid $\mathcal{M}$ and the full modular group $\mathrm{GL}(2, \mathbb{Z})$. We then describe various automorphic actions of $\mathcal{M}$.

In Section 3, the introduction of a complex analogue of the continued fraction expansion of a real number allows us to prove the convergence of the series (1.4) and (1.6) (Corollary 3.6). The main feature of the complex continued fraction is that it reduces to the real continued fraction when the number is real and it stops after a finite number of iterations when the number is rational or complex. In the latter case the absolute value of the imaginary part of the iterates grows at least exponentially with the number of iterations and when it reaches $1 / 2$ the iteration stops.

In Section 4 we use the complex continued fraction to study the behaviour of the series (1.4) when $z$ is close to $[0,1]$. This is interesting in itself and it will be very important when applied to the complex Brjuno function in order to prove our main results. Our study allows us to prove that the restriction of $T$ to the Hardy spaces $H^{p}(\overline{\mathbb{C}} \backslash[0,1]) \cap \mathcal{O}^{1}(\overline{\mathbb{C}} \backslash[0,1]), 1 \leq p \leq+\infty$, is continuous with spectral radius bounded above by $\frac{\sqrt{5}-1}{2}$. The same result holds also on the space of functions $\varphi \in \mathcal{O}^{1}(\overline{\mathbb{C}} \backslash[0,1])$ with bounded real part.

The complex Brjuno function is finally introduced in Section 5 where we state and prove our main results. 
In the Appendices we recall the results we need on the real continued fraction, on the hyperfunctions and on the dilogarithm. Then we show how to relate the complex Brjuno function with the even real Brjuno function treated in [MMY. Finally we describe how the real Brjuno function can be viewed as a cocycle under the action of the modular group.

\section{Modular group, the monoid $\mathcal{M}$ and its aCtion}

According to (1.4) the inversion of $(1-T)$ leads us to consider the monoid $\mathcal{M}$ of matrices of $\mathrm{GL}(2, \mathbb{Z})$. In this section we study its algebraic properties and we describe various actions of the modular group and of the monoid $\mathcal{M}$ on meromorphic or holomorphic functions.

\subsection{Algebraic properties, notation, structure of the monoid $\mathcal{M}$; relations} of $\mathcal{M}$ with the modular group and with Farey intervals.

\subsubsection{Notation.}

$G=\mathrm{GL}(2, \mathbb{Z})=\left\{\left(\begin{array}{ll}a & b \\ c & d\end{array}\right), a, b, c, d \in \mathbb{Z}, \varepsilon_{g}:=a d-b c= \pm 1\right\} ;$

$H$ is the subgroup of order 8 of matrices of the form $\left(\begin{array}{cc}\varepsilon & 0 \\ 0 & \varepsilon^{\prime}\end{array}\right)$ or $\left(\begin{array}{cc}0 & \varepsilon \\ \varepsilon^{\prime} & 0\end{array}\right)$, where $\varepsilon, \varepsilon^{\prime} \in\{-1,+1\} ;$

$\mathcal{M}$ is the monoid with unit $\left(\begin{array}{ll}1 & 0 \\ 0 & 1\end{array}\right)$ made of matrices $g=\left(\begin{array}{ll}a & b \\ c & d\end{array}\right) \in G$ such that, if $g \neq \mathrm{id}$, we have $d \geq b \geq a \geq 0$ and $d \geq c \geq a$.

$Z$ is the subgroup of matrices of the form $\left(\begin{array}{ll}1 & n \\ 0 & 1\end{array}\right), n \in \mathbb{Z}$.

2.1.2. Let $g(m)=\left(\begin{array}{cc}0 & 1 \\ 1 & m\end{array}\right)$, where $m \geq 1$. Clearly $g(m) \in \mathcal{M}$. Moreover, $\mathcal{M}$ is the free monoid generated by the elements $g(m), m \geq 1$ : each element $g$ of $\mathcal{M}$ can be written as $g=g\left(m_{1}\right) \cdots g\left(m_{r}\right), r \geq 0, m_{i} \geq 1$, and this decomposition is unique (see Proposition A1.2).

2.1.3. One has

$$
G=Z \cdot \mathcal{M} \cdot H
$$

i.e. the application $Z \times \mathcal{M} \times H \rightarrow G, \quad(z, m, h) \mapsto g=z \cdot m \cdot h$ is a bijection.

2.1.4. The subset $Z \cdot \mathcal{M}$ of $G$ is made of matrices $g=\left(\begin{array}{ll}a & b \\ c & d\end{array}\right)$ such that $d \geq c \geq 0$ with the following additional restrictions: $a=1$ if $c=0$, and $b=a+1$ if $d=c=1$.

We will also often use the following remark, which is an immediate consequence of the structure of $\mathcal{M}$ and of the relation

$$
g(m)\left(\begin{array}{ll}
1 & 1 \\
0 & 1
\end{array}\right)=g(m+1):
$$

one has the partition

$$
Z \cdot \mathcal{M}=Z \cdot \mathcal{M} \cdot\left(\begin{array}{ll}
1 & 1 \\
0 & 1
\end{array}\right) \sqcup Z \cdot \mathcal{M} \cdot\left(\begin{array}{ll}
0 & 1 \\
1 & 1
\end{array}\right)
$$


2.1.5. Let us consider the usual action of $G$ on $\overline{\mathbb{C}}=\mathbb{C} \cup\{\infty\}$ by homographies: $\left(\begin{array}{ll}a & b \\ c & d\end{array}\right) \cdot z=\frac{a z+b}{c z+d}$. The following facts are easy to check:

1. $g \cdot[0,1]=[0,1]$ if and only if $g$ belongs to the subgroup of order 4 of matrices of the form $\pm\left(\begin{array}{ll}1 & 0 \\ 0 & 1\end{array}\right), \pm\left(\begin{array}{cc}-1 & 1 \\ 0 & 1\end{array}\right)$

2. The monoid of the elements $g$ such that $g \cdot[0,1] \subset[0,1]$ admits the partition

$$
\mathcal{M} \sqcup \mathcal{M}\left(\begin{array}{cc}
-1 & 0 \\
0 & -1
\end{array}\right) \sqcup \mathcal{M}\left(\begin{array}{cc}
-1 & 1 \\
0 & 1
\end{array}\right) \sqcup \mathcal{M}\left(\begin{array}{cc}
1 & -1 \\
0 & -1
\end{array}\right) \text {. }
$$

Note that $\left(\begin{array}{cc}-1 & 1 \\ 0 & 1\end{array}\right)=\left(\begin{array}{ll}1 & 1 \\ 0 & 1\end{array}\right)\left(\begin{array}{cc}-1 & 0 \\ 0 & 1\end{array}\right)$.

3. The application $g \mapsto g \cdot 1=\frac{a+b}{c+d}$ is a bijection of $Z \mathcal{M}$ over $\mathbb{Q}$ which maps $\mathcal{M}$ onto $\mathbb{Q} \cap(0,1]$.

4. The application $g \mapsto g \cdot 0=b / d$ maps $Z \mathcal{M}$ onto $\mathbb{Q}$ and each rational number has exactly two inverse images. The two elements which map 0 on 1 are $\left(\begin{array}{ll}1 & 1 \\ 0 & 1\end{array}\right)$ and $\left(\begin{array}{ll}0 & 1 \\ 1 & 1\end{array}\right)$. This makes the partition of 2.1.4 less mysterious.

5. The application $g \mapsto g \cdot[0,+\infty]$ is a bijection of $Z \mathcal{M}$ on the set of Farey intervals (the convention we adopt here implies that $[n,+\infty]$ is a Farey interval, but $[-\infty, n]$ is not). For the definition and properties of the Farey partition of $[0,1]$ we refer the reader to $[\mathrm{HW}$.

6. The application $g \mapsto g \cdot \infty=a / c \in \overline{\mathbb{Q}}=\mathbb{Q} \cup\{\infty\}$ of $Z \mathcal{M}$ on $\overline{\mathbb{Q}}$ is surjective. Moreover

- $g \cdot \infty=\infty$ if and only if $g \in Z$;

- $g \cdot \infty=n \in \mathbb{Z}$ if and only if

$$
g=\left(\begin{array}{cc}
n & 1+k n \\
1 & k
\end{array}\right), \quad k \geq 1, \quad \text { or } \quad g=\left(\begin{array}{cc}
n & -1+k n \\
1 & k
\end{array}\right), k \geq 2 ;
$$

- $g \cdot \infty=a / c, c>1$, if and only if

$$
g=\left(\begin{array}{ll}
a & a^{\prime}+k a \\
c & c^{\prime}+k c
\end{array}\right), \quad k \geq 1, \quad \text { or } \quad g=\left(\begin{array}{ll}
a & a^{\prime \prime}+k a \\
c & c^{\prime \prime}+k c
\end{array}\right), \quad k \geq 1
$$

where $\left[\frac{a^{\prime}}{c^{\prime}}, \frac{a^{\prime \prime}}{c^{\prime \prime}}\right]$ is the Farey interval which contains $a / c$.

\subsection{Actions of $\mathcal{M}$ on some spaces of holomorphic functions.}

2.2.1. Let $U$ be an open subset of $\overline{\mathbb{C}}$. We will denote by $\mathcal{O}(U)$ the complex vector space of holomorphic functions on $U$. Let $I \subset \mathbb{R}$ be a compact interval and let $k \in \mathbb{Z}$. If $k \geq 0\left(k<0\right.$ respectively), we will denote with $\mathcal{O}^{k}(\overline{\mathbb{C}} \backslash I)$ the complex vector space of functions holomorphic in $\mathbb{C} \backslash I$, meromorphic in $\overline{\mathbb{C}} \backslash I$, which have a zero at infinity of order at least $k$ (resp. a pole of order at most $|k|)$.

Let $g=\left(\begin{array}{ll}a & b \\ c & d\end{array}\right) \in G$ and assume that $\varphi$ is meromorphic in $U$. We define

$$
L_{g}^{(k)} \varphi(z)=(a-c z)^{-k} \varphi\left(\frac{d z-b}{a-c z}\right)
$$


The function $L_{g}^{(k)} \varphi$ is meromorphic in $g \cdot U$. Note that

$$
\begin{aligned}
\left(L_{g}^{(k)} \varphi\right)^{\prime} & =k c L_{g}^{(k+1)} \varphi+\varepsilon_{g} L_{g}^{(k+2)} \varphi^{\prime}, \\
\left(L_{g}^{(k)} \varphi\right)^{\prime \prime} & =k(k+1) c^{2} L_{g}^{(k+2)} \varphi+\varepsilon_{g}(2 k+2) c L_{g}^{(k+3)} \varphi^{\prime}+L_{g}^{(k+4)} \varphi^{\prime \prime} ;
\end{aligned}
$$

thus, for $k=-1$

$$
\left(L_{g}^{(-1)} \varphi\right)^{\prime \prime}=L_{g}^{(3)} \varphi^{\prime \prime}
$$

Note also that if $g \in Z$, then $L_{g}^{(k)}$ does not depend on $k$.

The formula (2.1) above clearly defines an action of $G$ : if $g, g^{\prime} \in G, k \in \mathbb{Z}$ and $\varphi$ is meromorphic in $U$, then the functions $L_{g}^{(k)}\left(L_{g^{\prime}}^{(k)} \varphi\right)$ and $L_{g g^{\prime}}^{(k)} \varphi$ (meromorphic on $\left.g g^{\prime} \cdot U\right)$ coincide.

2.2.2. Let $J$ denote a compact interval of $\mathbb{R}$ and let $\varphi \in \mathcal{O}^{2}(\overline{\mathbb{C}} \backslash J)$. The series

$$
\sum_{n \in \mathbb{Z}} \varphi(z-n)=\sum_{g \in Z} L_{g}^{(k)} \varphi(\text { for all } k)
$$

converges uniformly on compact subsets of $\mathbb{C} \backslash \mathbb{R}$ and also on the domains $\{z \in$ $\mathbb{C},|\Im m z| \geq \delta>0,|\mathfrak{I} e z| \leq A\}$. The sum will be denoted $\sum_{z} \varphi$; it is a function holomorphic in $\mathbb{C} \backslash \mathbb{R}$, periodic of period 1 and vanishing at $\pm i \infty$. Thus taking the quotient by $\mathbb{Z}$ it can be represented by means of the variable $q=e^{ \pm 2 \pi i z}$.

If $\varphi \in \mathcal{O}^{1}(\overline{\mathbb{C}} \backslash J)$ with $[0,1] \subset J$, then one can decompose in a unique way

$$
\varphi(z)=a_{0} \log \frac{z}{z-1}+\varphi_{0}(z)
$$

where $a_{0} \in \mathbb{C}, \varphi_{0} \in \mathcal{O}^{2}(\overline{\mathbb{C}} \backslash J)$ and we consider the main branch of the logarithm in $\mathbb{C} \backslash \mathbb{R}^{-}$. We have

$$
\sum_{n=-N}^{N} \log \frac{z-n}{z-n-1}=\log \frac{z+N}{z-N-1}
$$

and this leads to the definition

$$
\sum_{Z} \varphi(z):=\sum_{Z} \varphi_{0}(z)+ \begin{cases}-a_{0} \pi i & \text { if } \mathfrak{I} m z>0 \\ +a_{0} \pi i & \text { if } \mathfrak{I} m z<0\end{cases}
$$

Note that in order to insure the convergence of the series $\sum_{g \in Z} L_{g}^{(k)} \varphi$ one must regroup together the terms $\left(\begin{array}{ll}1 & n \\ 0 & 1\end{array}\right)$ and $\left(\begin{array}{cc}1 & -n \\ 0 & 1\end{array}\right), n \geq 1$ (symmetric summation or Eisenstein's summation).

2.2.3. Let $k \in \mathbb{Z}$ and $g \in \mathcal{M}$. Since $g \cdot[0,1] \subset[0,1]$ if $\varphi$ is meromorphic in $\overline{\mathbb{C}} \backslash[0,1]$, then $L_{g}^{(k)} \varphi$ will still be meromorphic in $\overline{\mathbb{C}} \backslash[0,1]$. Moreover if $\varphi \in \mathcal{O}^{k}(\overline{\mathbb{C}} \backslash[0,1])$, then also $L_{g}^{(k)} \varphi \in \mathcal{O}^{k}(\overline{\mathbb{C}} \backslash[0,1])$. Thus one has an action of the monoid $\mathcal{M}$ on $\mathcal{O}^{k}(\overline{\mathbb{C}} \backslash[0,1])$. 
2.2.4. We will now define a new action $L_{g}$ of $\mathcal{M}$ on $\mathcal{O}^{1}(\overline{\mathbb{C}} \backslash[0,1])$ which differs from the action $L_{g}^{(-1)}$ on $\mathcal{O}^{-1}(\overline{\mathbb{C}} \backslash[0,1])$ by an affine correction.

All functions $\varphi \in \mathcal{O}^{-1}(\overline{\mathbb{C}} \backslash[0,1])$ can be uniquely written as

$$
\varphi(z)=A z+B+p(\varphi)(z), \quad p(\varphi) \in \mathcal{O}^{1}(\overline{\mathbb{C}} \backslash[0,1]) .
$$

Note that if $g=\left(\begin{array}{ll}a & b \\ c & d\end{array}\right) \in G$ and $\psi(z)=A z+b$, then

$$
L_{g}^{(-1)} \psi(z)=A(d z-b)+B(a-c z)
$$

is still an affine function. Thus the formula

$$
L_{g} \varphi:=p\left(L_{g}^{(-1)} \varphi\right) \text {, }
$$

where $g \in \mathcal{M}, \varphi \in \mathcal{O}^{1}(\overline{\mathbb{C}} \backslash[0,1])$, defines an action of $\mathcal{M}$ on $\mathcal{O}^{1}(\overline{\mathbb{C}} \backslash[0,1])$ which makes the following diagram commute:

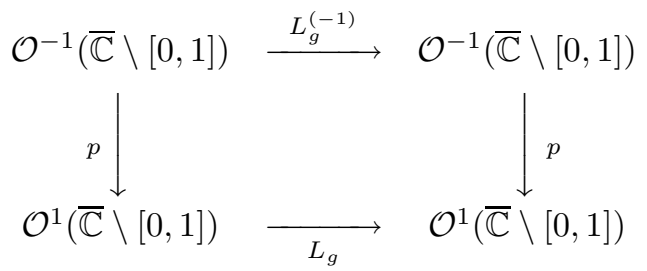

More explicitly, if $g=\left(\begin{array}{ll}a & b \\ c & d\end{array}\right) \in \mathcal{M}$, then

$$
L_{g} \varphi(z)=(a-c z)\left[\varphi\left(\frac{d z-b}{a-c z}\right)-\varphi\left(-\frac{d}{c}\right)\right]-\varepsilon_{g} c^{-1} \varphi^{\prime}\left(-\frac{d}{c}\right) .
$$

Since this definition differs from that of $L_{g}^{(-1)}$ only by an affine correction one clearly has

$$
\left(L_{g} \varphi\right)^{\prime \prime}=L_{g}^{(3)} \varphi^{\prime \prime},
$$

where $\varphi^{\prime \prime} \in \mathcal{O}^{3}(\overline{\mathbb{C}} \backslash[0,1])$. Equivalently one can say that $L_{g} \varphi$ is obtained by taking the double primitive of $L_{g}^{(3)} \varphi^{\prime \prime}$ which vanishes at infinity.

Two other formulas will be used throughout what follows: if $\varphi \in \mathcal{O}^{1}(\overline{\mathbb{C}} \backslash[0,1])$, $g=\left(\begin{array}{ll}a & b \\ c & d\end{array}\right) \in \mathcal{M}$ and $z \notin\left[\frac{b}{d}, \frac{a}{c}\right]=g \cdot[0,+\infty]$, one has

$$
\begin{aligned}
L_{g} \varphi(z) & =\varepsilon_{g} c^{-1}\left[\int_{0}^{1} \varphi^{\prime}\left(-\frac{d}{c}+\varepsilon_{g} \frac{t}{c(a-c z)}\right) d t-\varphi^{\prime}\left(-\frac{d}{c}\right)\right] \\
& =c^{-2}(a-c z)^{-1} \int_{0}^{1} \varphi^{\prime \prime}\left(-\frac{d}{c}+\varepsilon_{g} \frac{t}{c(a-c z)}\right)(1-t) d t .
\end{aligned}
$$

Note that the assumption about $z$ means that the segment whose extremities are $-d / c$ and $(d z-b) /(a-c z)$ does not intersect the interval [0,1]. The two formulas are thus nothing else than Taylor's formulas of first and second order with integral remainder.

2.2.5. If $\varphi \in \mathcal{O}(\overline{\mathbb{C}} \backslash I)$, we denote $\sigma \cdot \varphi(z)=\overline{\varphi(\bar{z})} . \sigma$ is an involution of $\mathcal{O}(\overline{\mathbb{C}} \backslash I)$ which preserves all the subspaces $\mathcal{O}^{k}(\overline{\mathbb{C}} \backslash I)$, commutes with $p$ and with the actions of $\mathcal{M}$ on $\mathcal{O}^{k}$. If $\sigma \cdot \varphi=\varphi$, then $\varphi(x) \in \mathbb{R}$ for all $x \in \mathbb{R} \backslash I$, and we say that $\varphi$ is real holomorphic. 


\section{Complex CONTINued FraCtions}

Exactly as in the real case treated in [MMY, where the use of the continued fraction expansion was important for the study of the real Brjuno function, the introduction of a complex analogue of Gauss' algorithm of continued fraction expansion of a real number will be essential for the study of the boundary behaviour of $\sum_{\mathcal{M}}\left(L_{g} \varphi\right)(z)$ and the construction of the complex Brjuno function. In this section we first define our complex version of the continued fraction (Section 3.1) then we use it to estimate the spectral radius of $T$ (Section 3.2) and to prove the convergence of the series (1.4) and (1.6) (Corollary 3.6).

\subsection{Definition of the complex continued fractions.}

3.1.1. We consider the following domains:

$$
\begin{aligned}
D_{0} & =\left\{z \in \mathbb{C},|z+1| \leq 1, \mathfrak{R} e z \geq \frac{\sqrt{3}}{2}-1\right\}, \\
D_{1} & =\left\{z \in \mathbb{C},|z| \geq 1,\left|z-\frac{1}{\sqrt{3}}\right| \leq \frac{1}{\sqrt{3}}\right\}, \\
D & =\{z \in \mathbb{C},|z| \leq 1,|z-i| \geq 1,|z+i| \geq 1, \mathfrak{R} e z>0\}, \\
H_{0} & =\{z \in \mathbb{C},|z-i| \leq 1,|z+1| \geq 1, \mathfrak{I} m z \leq 1 / 2\}, \\
H_{0}^{\prime} & =\left\{z \in \mathbb{C}, \bar{z} \in H_{0}\right\}, \\
\Delta & =H_{0} \cup H_{0}^{\prime} \cup D=\{z \in \mathbb{C},|z| \leq 1,|z+1| \geq 1,|\mathfrak{I} m z| \leq 1 / 2\}, \\
D_{\infty} & =\overline{\mathbb{C}} \backslash\left(D_{0} \cup \Delta \cup D_{1}\right) \\
& =\{|\mathfrak{I} m z|>1 / 2\} \cup\left\{\mathfrak{R} e z<\frac{\sqrt{3}}{2}-1\right\} \cup\left\{\mathfrak{R} e z>\frac{\sqrt{3}}{2},|z-1 / \sqrt{3}|>1 / \sqrt{3}\right\} .
\end{aligned}
$$

Figures 2 and 3 show these domains and their image under the inversion $S(z)=1 / z$. A fundamental property is the following:

- if $z \notin D \cup D_{1}$ (in particular if $z \in D_{\infty}$ ), then $1 / z-m \in D_{\infty}$ for all $m \geq 1$;

- if $z \in D_{1}$, then $1 / z-1 \in D_{0}$ and $1 / z-m \in D_{\infty}$ for all $m \geq 2$.

Observe that $S D=\bigcup_{m \geq 1}(\Delta+m)$, where the domains have disjoint interior. Thus, for $z \in D$, we define

$$
A(z)=\frac{1}{z}-m=(g(m))^{-1} \cdot z
$$

(we recall that $g(m)=\left(\begin{array}{cc}0 & 1 \\ 1 & m\end{array}\right), m \geq 1$ ), where $m \geq 1$ is the unique integer such that $A(z) \in \Delta,|A(z)|<1$. Iterating from $z_{0} \in D$, we define

$$
z_{i+1}=A\left(z_{i}\right)=A^{i+1}\left(z_{0}\right)
$$

as long as $z_{i}=A^{i}(z) \in D$. The iteration process stops when one of the two following conditions is verified:

- $z_{l}=0$ for some $l \geq 0$; this happens if and only if $z_{0} \in \mathbb{Q}$,

- $z_{l} \notin(D \cup\{0\})$ for some $l \geq 0$; this happens if and only if $z_{0} \notin \mathbb{R}$.

For all $0 \leq i<l$, we will denote the integer $m_{i+1}$ such that

$$
z_{i+1}=\frac{1}{z_{i}}-m_{i+1}, \quad m_{i+1} \geq 1 .
$$




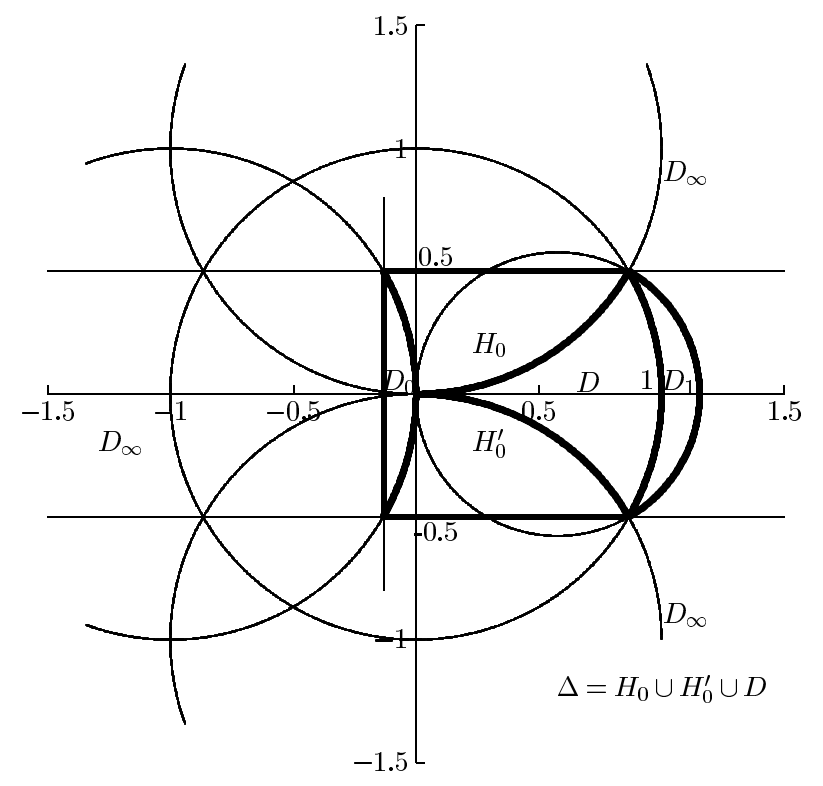

Figure 2. The various domains $D_{i}$ used for the definition of the complex continued fraction.

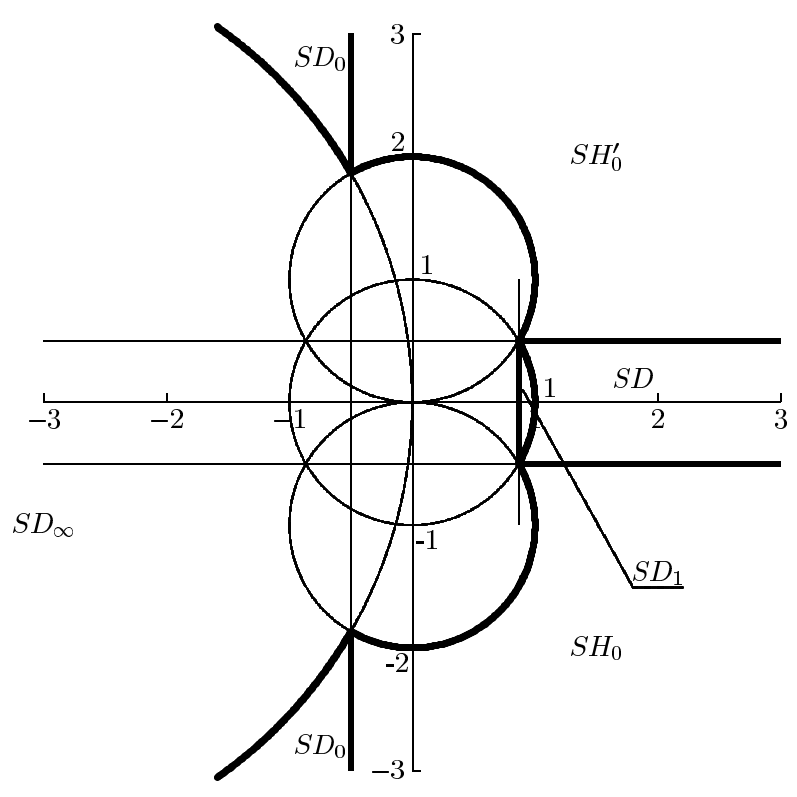

Figure 3. Result of the action of $S: z \mapsto 1 / z$ on the domains of Figure 2 . 
3.1.2. Let $\left(\begin{array}{cc}p_{i-1} & p_{i} \\ q_{i-1} & q_{i}\end{array}\right)=\left(\begin{array}{cc}0 & 1 \\ 1 & m_{1}\end{array}\right) \ldots\left(\begin{array}{cc}0 & 1 \\ 1 & m_{i}\end{array}\right) \in \mathcal{M}, 0 \leq i \leq l$. Then one has the same recurrence relations as for the real continued fraction

$$
\begin{aligned}
p_{i+1} & =m_{i+1} p_{i}+p_{i-1}, \\
q_{i+1} & =m_{i+1} q_{i}+q_{i-1},
\end{aligned}
$$

with initial data $p_{-1}=q_{0}=1$ and $p_{0}=q_{-1}=0$. Moreover

$$
z_{0}=\frac{p_{i-1} z_{i}+p_{i}}{q_{i-1} z_{i}+q_{i}}, \quad z_{i}=\frac{p_{i}-q_{i} z_{0}}{q_{i-1} z_{0}-p_{i-1}}
$$

and if one poses

$$
\beta_{i}\left(z_{0}\right)=\prod_{j=0}^{i} z_{j}=(-1)^{i}\left(q_{i} z_{0}-p_{i}\right)
$$

then

$$
\beta_{i}\left(z_{0}\right)=\frac{z_{i}}{q_{i}+q_{i-1} z_{i}}=\frac{1}{q_{i+1}+q_{i} z_{i+1}}
$$

Finally one has

$$
\begin{aligned}
(-1)^{i} \mathfrak{I} m z_{0} & =\left|\beta_{i-1}\left(z_{0}\right)\right|^{2} \mathfrak{I} m z_{i}=\left|q_{i}+q_{i-1} z_{i}\right|^{-2} \mathfrak{I} m z_{i} \\
\frac{d z_{i}}{d z_{0}} & =(-1)^{i}\left(\beta_{i-1}\left(z_{0}\right)\right)^{-2}=(-1)^{i}\left(q_{i}+q_{i-1} z_{i}\right)^{2} .
\end{aligned}
$$

Observe that, as $\left|z_{i+1}+1\right| \geq 1$ and $\mathfrak{R} e z_{i+1} \geq \frac{\sqrt{3}}{2}-1$ for $i<l$, we have from (3.7)

$$
\left|\beta_{i}\left(z_{0}\right)\right| \leq q_{i+1}^{-1}[\cos \pi / 12]^{-1}=\frac{2 \sqrt{2}}{1+\sqrt{3}} q_{i+1}^{-1}
$$

and, as $q_{i} \leq q_{i+1},\left|z_{i+1}\right| \leq 1$,

$$
\left|\beta_{i}\left(z_{0}\right)\right| \geq \frac{1}{2} q_{i+1}^{-1}
$$

\subsection{The operator $T$, its spectral radius and the sum over the monoid.}

3.2.1. Let $\gamma_{1}>\gamma_{0}>-1, I=\left[\gamma_{0}, \gamma_{1}\right], \varphi \in \mathcal{O}^{1}(\overline{\mathbb{C}} \backslash I)$.

For all $m \geq 1$ one has (compare with (2.10))

$$
\begin{aligned}
L_{g(m)} \varphi(z) & =-z\left(\varphi\left(\frac{1}{z}-m\right)-\varphi(-m)\right)+\varphi^{\prime}(-m) \\
& =-z^{-1} \int_{0}^{1} \varphi^{\prime \prime}\left(-m+\frac{t}{z}\right)(1-t) d t
\end{aligned}
$$

provided that $z \notin\left[0,1 /\left(\gamma_{0}+m\right)\right]$, and one has an even simpler formula for the action at the level of second derivatives

$$
L_{g(m)}^{(3)} \psi(z)=-z^{-3} \psi\left(\frac{1}{z}-m\right)
$$


3.2.2. Let $\varepsilon>0$,

$$
U_{\varepsilon}=\left\{z \in \mathbb{C}, \mathfrak{R} e z \leq \gamma_{0}-\varepsilon \text { or } \mathfrak{R} e z \geq \gamma_{1}+\varepsilon \text { or }|\Im m z| \geq \varepsilon\right\} .
$$

We have the following

Proposition 3.1. Let $\gamma_{0}, \gamma_{1}$ be as above, $I=\left[\gamma_{0}, \gamma_{1}\right], J=\left[0,1 /\left(1+\gamma_{0}\right)\right]$.

1) For all $\varphi \in \mathcal{O}^{1}(\overline{\mathbb{C}} \backslash I)$, the series $\sum_{m \geq 1} L_{g(m)} \varphi$ converges uniformly on compact subsets $K$ of $\overline{\mathbb{C}} \backslash J$ to a function $T \varphi \in \mathcal{O}^{1}(\overline{\mathbb{C}} \backslash J)$ and there exist $\varepsilon>0$ and $C_{K}>0$ such that $\sup _{K}|T \varphi| \leq C_{K} \sup _{U_{\varepsilon}}|\varphi|$.

2) For all $\psi \in \mathcal{O}^{3}(\overline{\mathbb{C}} \backslash I)$ the series $\sum_{m \geq 1} L_{g(m)}^{(3)} \psi$ converges uniformly on compact subsets $K$ of $\overline{\mathbb{C}} \backslash J$ to a function $T^{(3)} \psi \in \mathcal{O}^{1}(\overline{\mathbb{C}} \backslash J)$ and there exist $\varepsilon>0$ and $C_{K}>0$ such that $\sup _{K}\left|T^{(3)} \psi\right| \leq C_{K} \sup _{U_{\varepsilon}}|\psi|$.

3) For all $\varphi \in \mathcal{O}^{1}(\overline{\mathbb{C}} \backslash I)$ one has $T^{(3)} \varphi^{\prime \prime}=(T \varphi)^{\prime \prime}$.

Proof. Let $\varepsilon>0$. There exists $c_{\varepsilon}>0$ such that for $\psi \in \mathcal{O}^{3}(\overline{\mathbb{C}} \backslash I), z \in U_{\varepsilon}$ one has

$$
|\psi(z)| \leq c_{\varepsilon}|z|^{-3} \sup _{U_{\varepsilon}}|\psi| \text {. }
$$

If $K$ is a compact subset of $\overline{\mathbb{C}} \backslash J$, there exists $\varepsilon=\varepsilon_{K}$ such that $1 / z-m \in U_{\varepsilon}$ for all $m \geq 1$ and $z \in K$. Moreover there exist $c_{K}>0$ and $M=M\left(\gamma_{0}\right)$, such that $|1 / z-m|^{-1} \leq c_{K} m^{-1}$ for all $z \in K, m \geq M$. Consequently, for $z \in K$ and $\psi \in \mathcal{O}^{3}(\overline{\mathbb{C}} \backslash I)$ we have

$$
\sum_{m \geq 1}\left|\psi\left(\frac{1}{z}-m\right)\right| \leq c_{K}^{\prime} \sup _{U_{\varepsilon}}|\psi|, \quad \text { with } \quad c_{K}^{\prime}=M+c_{\varepsilon} c_{K}^{3} \sum_{m \geq M} m^{-3},
$$

which proves the second part of the proposition.

By integrating twice from $\infty$ one deduces that for $\varphi \in \mathcal{O}^{1}(\overline{\mathbb{C}} \backslash I)$ the series $\sum_{m \geq 1} L_{g(m)} \varphi$ converges uniformly on compact subsets of $\overline{\mathbb{C}} \backslash J$ to a function $T \varphi \in$ $\mathcal{O}^{1}(\overline{\mathbb{C}} \backslash J)$. Moreover for any compact $K \subset \overline{\mathbb{C}} \backslash J$ there exist $c_{\varepsilon}>0$ and $c_{K}>0$ such that for $z \in K,\left|(T \varphi)^{\prime \prime}(z)\right|=\left|\left(T^{(3)} \varphi^{\prime \prime}\right)(z)\right| \leq c_{K}|z|^{-3} \sup _{U_{\varepsilon}}\left|\varphi^{\prime \prime}\right|$. On the other hand there exists $c_{\varepsilon}^{\prime \prime}>0$ such that $\sup _{U_{\varepsilon}}\left|\varphi^{\prime \prime}\right| \leq c_{\varepsilon}^{\prime \prime} \sup _{U_{\varepsilon / 2}}|\varphi|$ (by Cauchy's formula), hence we get for $z \in K,|T \varphi(z)| \leq \tilde{c}_{K}|z|^{-1} \sup _{U_{\varepsilon / 2}}|\varphi|$. The third part of the proposition is immediate.

3.2.3. The open set $\overline{\mathbb{C}} \backslash[0,1]$ is a hyperbolic Riemann surface which is naturally equipped with a Poincaré metric. The following well-known fact will be crucial for the proof of Lemma 3.2: given two hyperbolic Riemann surfaces $M, N$ and an analytic map $f: M \rightarrow N$, either its differential $d f$ contracts the hyperbolic metric or $f$ is a surjective local isometry. In what follows we will denote by $d_{\text {hyper }}$ the Poincaré metric on the Riemann surface under consideration.

Given $\rho>0$ we denote by

$$
V_{\rho}\left(D_{\infty}\right)=\left\{z \in \overline{\mathbb{C}} \backslash[0,1], d_{\text {hyper }}\left(z, D_{\infty}\right)<\rho\right\}
$$

the $\rho$-neighborhood of $D_{\infty}$ in $\overline{\mathbb{C}} \backslash[0,1]$.

Lemma 3.2. Let $\rho \geq 0$. For all $m \geq 1$ and $z \in V_{\rho}\left(D_{\infty}\right)$ one has $\frac{1}{z}-m \in V_{\rho}\left(D_{\infty}\right)$.

Proof. The Möbius transformation $z \mapsto \frac{1}{z}-m$ maps $D_{\infty}$ into itself and $\overline{\mathbb{C}} \backslash[0,1]$ onto $\overline{\mathbb{C}} \backslash[1-m,+\infty]$ which is contained in $\overline{\mathbb{C}} \backslash[0,1]$. Thus it decreases the hyperbolic distance and the $\rho$-neighborhood of the image of $D_{\infty}$ w.r.t. the Poincaré metric 
of $\overline{\mathbb{C}} \backslash[1-m,+\infty]$ is contained in the $\rho$-neighborhood of $D_{\infty}$ w.r.t. the Poincaré metric of $\overline{\mathbb{C}} \backslash[0,1]$.

3.2.4. Once the existence of the operator $T$ on holomorphic functions is established (Proposition 3.1) one can ask for more information on Hardy spaces. The results we will prove are completely analogous to those obtained for the real Brjuno operator in MMY].

Proposition 3.3. Let $\rho \geq 0$. There exists $c_{\rho}^{\prime}>0$ such that for all $r \geq 0$ and $\psi \in \mathcal{O}^{3}(\overline{\mathbb{C}} \backslash[0,1])$ one has

$$
\sup _{V_{\rho}\left(D_{\infty}\right)}\left|\left(\left(T^{(3)}\right)^{r} \psi\right)(z)\right| \leq c_{\rho}^{\prime}\left(\frac{\sqrt{5}-1}{2}\right)^{r} \sup _{V_{\rho}\left(D_{\infty}\right)}|\psi(z)| .
$$

Proof. First of all note that if $z \in D_{\infty}$ and $g \in \mathcal{M}$, then $g^{-1} \cdot z \in D_{\infty}$; thus, taking into account Lemma 3.2, if $z \in V_{\rho}\left(D_{\infty}\right)$, then $g^{-1} z \in V_{\rho}\left(D_{\infty}\right)$.

Given an integer $r \geq 0$ we denote by $\mathcal{M}^{(r)}$ the set of elements $g$ of $\mathcal{M}$ of the form $g\left(m_{1}\right) \ldots g\left(m_{r}\right), m_{i} \geq 1$ for all $1 \leq i \leq r$. Then one has

$$
\left(T^{(3)}\right)^{r} \psi=\sum_{g \in \mathcal{M}^{(r)}} L_{g}^{(3)} \psi=\sum_{g^{\prime} \in \mathcal{M}^{(r-1)}} L_{g^{\prime}}^{(3)}\left(T^{(3)} \psi\right) .
$$

Let $g^{\prime}=\left(\begin{array}{ll}a^{\prime} & b^{\prime} \\ c^{\prime} & d^{\prime}\end{array}\right) \in \mathcal{M}^{(r-1)}$ and $z \in V_{\rho}\left(D_{\infty}\right)$; let $z^{\prime}=\frac{d^{\prime} z-b^{\prime}}{a^{\prime}-c^{\prime} z}$. For all $m \geq 1$ one has

$$
\left(\begin{array}{ll}
a^{\prime} & b^{\prime} \\
c^{\prime} & d^{\prime}
\end{array}\right)\left(\begin{array}{cc}
0 & 1 \\
1 & m
\end{array}\right)=\left(\begin{array}{cc}
b^{\prime} & d^{\prime} \\
a^{\prime}+m b^{\prime} & c^{\prime}+m d^{\prime}
\end{array}\right)
$$

from which it follows that

$$
L_{g^{\prime}}^{(3)}\left(T^{(3)} \psi\right)(z)=\left(b^{\prime}-d^{\prime} z\right)^{-3} \sum_{m \geq 1} \psi\left(\frac{1}{z^{\prime}}-m\right) .
$$

Since $z^{\prime} \in V_{\rho}\left(D_{\infty}\right)$, as we have seen during the proof of Proposition 3.1 one has

$$
\left|\sum_{m \geq 1} \psi\left(\frac{1}{z^{\prime}}-m\right)\right| \leq c_{\rho} \sup _{V_{\rho}\left(D_{\infty}\right)}|\psi| \text {. }
$$

On the other hand one has $\left|z-b^{\prime} / d^{\prime}\right|^{-1} \leq c_{\rho}$ for all $z \in V_{\rho}\left(D_{\infty}\right)$ and $b^{\prime} / d^{\prime} \in[0,1]$. Thus we get $\left|L_{g^{\prime}}^{(3)}\left(T^{(3)} \psi\right)(z)\right| \leq \tilde{c}_{\rho}\left(d^{\prime}\right)^{-3}$. But now it is enough to recall that (see Appendix A1) $\min _{\mathcal{M}^{(r-1)}} d^{\prime} \geq C\left(\frac{\sqrt{5}+1}{2}\right)^{r-1}$ and $\sum_{\mathcal{M}^{(r-1)}} d^{\prime-2} \leq C$ to obtain the desired estimate.

Remark 3.4. In a completely analogous way we may prove that for $\rho \geq 0$ and all $\varphi \in \mathcal{O}^{1}(\overline{\mathbb{C}} \backslash[0,1])$,

$$
\sup _{V_{\rho}\left(D_{\infty}\right)}\left|T^{r} \varphi(z)\right| \leq c_{\rho}^{\prime}\left(\frac{\sqrt{5}-1}{2}\right)^{r} \sup _{V_{\rho}\left(D_{\infty}\right)}|\varphi(z)| .
$$

Remark 3.5. We may also consider the Hardy space $H^{p}\left(D_{\infty}\right), 1 \leq p<+\infty$, of analytic functions $\varphi: D_{\infty} \rightarrow \mathbb{C}$ such that the subharmonic function $|\varphi|^{p}$ has a harmonic majorant. It is an immediate consequence of the Riemann mapping 
theorem that this space is isomorphic to $H^{p}(\mathbb{D})$. Indeed if $h$ maps $D_{\infty}$ conformally onto $\mathbb{D}$ one can use the norm

$$
\|\varphi\|_{H^{p}\left(D_{\infty}\right)}=\left\|\varphi \circ h^{-1}\right\|_{H^{p}(\mathbb{D})}=\left(\int_{\partial D_{\infty}}|\varphi(z)|^{p}\left|h^{\prime}(z) \| d z\right|\right)^{1 / p} .
$$

Note that since $\partial D_{\infty}$ is a rectifiable Jordan curve, $h$ extends to a homeomorphism of $\partial D_{\infty}$ onto $\mathbb{S}^{1}$ which is conformal almost everywhere. It is immediate to check that the proof of Proposition 3.3 can be easily adapted so as to show that $T$ is a bounded linear operator on $H^{p}\left(D_{\infty}\right)$ with spectral radius $\leq \frac{\sqrt{5}-1}{2}$.

3.2.5. We now have the following important corollary, which establishes the convergence of the series (1.4).

Corollary 3.6. 1) Let $\psi \in \mathcal{O}^{3}(\overline{\mathbb{C}} \backslash[0,1])$. The family $\left(L_{g}^{(3)} \psi\right)_{g \in \mathcal{M}}$ is summable, uniformly on compact subsets of $\overline{\mathbb{C}} \backslash[0,1]$. Its sum is equal to $\sum_{r \geq 0}\left(T^{(3)}\right)^{r} \psi$, will be denoted $\sum_{\mathcal{M}}^{(3)} \psi$ and for all compact subsets $K$ of $\overline{\mathbb{C}} \backslash[0,1]$ there exists $\varepsilon>0$ such that

$$
\sup _{K}\left|\sum_{\mathcal{M}}^{(3)} \psi\right| \leq C_{K} \sup _{U_{\varepsilon}}|\psi|
$$

The family $\left(L_{g}^{(3)} \psi\right)_{g \in Z \cdot \mathcal{M}}$ is summable, uniformly on all domains of the form $\{|\Re e z|<A,|\mathfrak{I} m z| \geq \delta\}$ (where $A$ and $\delta$ are positive). Its sum is equal to $\sum_{\mathbb{Z}} \sum_{\mathcal{M}}^{(3)} \psi$ and will be denoted $\sum_{Z \cdot \mathcal{M}}^{(3)} \psi$. It is holomorphic in $\mathbb{C} \backslash \mathbb{R}$, periodic of period 1 and bounded in a neighborhood of $\pm i \infty$.

2) Let $\varphi \in \mathcal{O}^{1}(\overline{\mathbb{C}} \backslash[0,1])$. The family $\left(L_{g} \varphi\right)_{g \in \mathcal{M}}$ is summable, uniformly on compact subsets of $\overline{\mathbb{C}} \backslash[0,1]$. Its sum is equal to $\sum_{r \geq 0} T^{r} \varphi$ and will be denoted $\sum_{\mathcal{M}} \varphi$. The function $\sum_{\mathbb{Z}}\left(\sum_{\mathcal{M}} \varphi\right)$ will be denoted $\sum_{Z \cdot \mathcal{M}} \varphi$. It is holomorphic in $\mathbb{C} \backslash \mathbb{R}$, periodic of period 1 and vanishes at $\pm i \infty$. One has

$$
\left(\sum_{\mathcal{M}} \varphi\right)^{\prime \prime}=\sum_{\mathcal{M}}^{(3)} \varphi^{\prime \prime}, \quad\left(\sum_{Z \cdot \mathcal{M}} \varphi\right)^{\prime \prime}=\sum_{Z \cdot \mathcal{M}}^{(3)} \varphi^{\prime \prime}
$$

3) $\sum_{\mathcal{M}}\left(\right.$ resp. $\left.\sum_{\mathcal{M}}^{(3)}\right)$ and $(1-T)\left(\right.$ resp. $\left.\left(1-T^{(3)}\right)\right)$ acting on $\mathcal{O}^{1}(\overline{\mathbb{C}} \backslash[0,1])$ (resp. $\left.\mathcal{O}^{3}(\overline{\mathbb{C}} \backslash[0,1])\right)$ are the inverses of one another:

$$
\begin{gathered}
(1-T) \sum_{\mathcal{M}}=\sum_{\mathcal{M}}(1-T)=i d, \\
\left(1-T^{(3)}\right) \sum_{\mathcal{M}}^{(3)}=\sum_{\mathcal{M}}^{(3)}\left(1-T^{(3)}\right)=i d .
\end{gathered}
$$

Proof. 1) The only non-trivial assertions are the summability of the families $\left(L_{g}^{(3)} \psi\right)_{g \in \mathcal{M}}$ and $\left(L_{g}^{(3)} \psi\right)_{g \in Z \mathcal{M}}$. Writing $g=g^{\prime} g(m)\left(m \geq 1, g^{\prime} \in \mathcal{M}\right)$ for $g \in \mathcal{M}$, $g \neq$ id, we have

$$
L_{g}^{(3)} \psi(z)=\left(b^{\prime}-d^{\prime} z\right)^{-3} \psi\left(\frac{1}{z^{\prime}}-m\right)
$$

with

$$
g^{\prime}=\left(\begin{array}{ll}
a^{\prime} & b^{\prime} \\
c^{\prime} & d^{\prime}
\end{array}\right), \quad z^{\prime}=\frac{d^{\prime} z-b^{\prime}}{a^{\prime}-c^{\prime} z}
$$

Now for $z \in V_{\rho}\left(D_{\infty}\right),\left|L_{g}^{(3)} \psi(z)\right|=c_{\rho}\left(m d^{\prime} \operatorname{dist}(z,[0,1])\right)^{-3} \sup _{V_{\rho}\left(D_{\infty}\right)}|\psi|$, and the summability assertions follow (see the proof of Proposition 3.3). 
2) Again, the only non-trivial assertion is the summability one, which is obtained from the first part by integrating twice.

3) The third part of the corollary is immediate.

\section{Boundary Behaviour of $\sum_{\mathcal{M}} \varphi$}

In this section we will study the behaviour of $\sum_{\mathcal{M}} \varphi(z)$ when $z$ is close to $[0,1]$. Our main tool for this study will be the complex continued fraction introduced in the previous section. In this section and in the next one we will for shortness often denote by $c$ or $C$ various positive universal constants.

\subsection{Decomposition into principal and residual terms.}

4.1.1. We begin our study of the boundary behaviour of $\sum_{\mathcal{M}} \varphi(z)$ by considering the case when $z$ is close to 0 .

Proposition 4.1. 1) Let $I=\left[\gamma_{0}, \gamma_{1}\right], \gamma_{0}>-1$. There exists $c=c_{I}>0$ such that for all $\varphi \in \mathcal{O}^{1}(\overline{\mathbb{C}} \backslash I)$ and for all $z \in D_{0} \cup H_{0} \cup H_{0}^{\prime}$ one has

$$
\left|T \varphi(z)-\sum_{m \geq 1} \varphi^{\prime}(-m)\right| \leq c_{I}|z| \log \left(1+|z|^{-1}\right) \sup _{U}|\varphi|,
$$

where $U=\left\{z \in \mathbb{C},|\Im m z| \geq 1 / 2\right.$ or $\mathfrak{R} e z \leq \gamma_{0}-1$ or $\left.\mathfrak{R} e z \geq \gamma_{1}+1\right\}$.

2) There exists $c>0$ such that for all $\varphi \in \mathcal{O}^{1}(\overline{\mathbb{C}} \backslash[0,1])$ and for all $z \in D_{0} \cup$ $H_{0} \cup H_{0}^{\prime}$ one has

$$
\left|\sum_{\mathcal{M}} \varphi(z)-\varphi(z)-\sum_{m \geq 1}\left(\sum_{\mathcal{M}} \varphi\right)^{\prime}(-m)\right| \leq c|z|\left(1+\log |z|^{-1}\right) \sup _{D_{\infty}}|\varphi| .
$$

Proof. We will only prove (4.2), since the proof of (4.1) is essentially the same.

Let $\tilde{\varphi}=\sum_{\mathcal{M}} \varphi$. One has

$$
\tilde{\varphi}=\varphi+T \tilde{\varphi}=\varphi+\sum_{m \geq 1} L_{g(m)} \tilde{\varphi} .
$$

Let $z \in D_{0} \sqcup H_{0} \sqcup H_{0}^{\prime}$. We will consider the cases $m \geq 3|z|^{-1}$ and $m<3|z|^{-1}$ separately.

If $m \geq 3|z|^{-1}$ the segment $\left[-m,-m+\frac{1}{z}\right]$ is contained in the closed half plane $\{\Re$ e $w \leq-2 / 3\}$ and one has

$$
L_{g(m)} \tilde{\varphi}(z)=-\frac{1}{z} \int_{0}^{1}(1-t) \tilde{\varphi}^{\prime \prime}\left(-m+\frac{t}{z}\right) d t .
$$

Applying Cauchy's estimate, it follows that $\left|L_{g(m)} \tilde{\varphi}(z)\right| \leq c|z|^{-1} m^{-3} \sup _{D_{\infty}}|\tilde{\varphi}|$. Since by Remark $3.4 \sup _{D_{\infty}}|\tilde{\varphi}| \leq c \sup _{D_{\infty}}|\varphi|$ one gets

$$
\left|\sum_{m \geq 3|z|^{-1}} L_{g(m)} \tilde{\varphi}(z)\right| \leq c|z| \sup _{D_{\infty}}|\varphi| .
$$

In the case $m<3|z|^{-1}$ we separate the three terms constituting $L_{g(m)} \tilde{\varphi}(z)$ and we obtain

$$
\begin{gathered}
\left|\tilde{\varphi}^{\prime}(-m)\right| \leq c m^{-2} \sup _{D_{\infty}}|\tilde{\varphi}|, \quad|z \tilde{\varphi}(-m)| \leq c m^{-1}|z| \sup _{D_{\infty}}|\tilde{\varphi}| \\
\left|z \tilde{\varphi}\left(-m+\frac{1}{z}\right)\right| \leq c\left|-m+\frac{1}{z}\right|^{-1} \sup _{D_{\infty}}|\tilde{\varphi}|
\end{gathered}
$$


thus

$$
\left|\sum_{m<3|z|^{-1}} L_{g(m)} \tilde{\varphi}(z)-\sum_{m \geq 1} \tilde{\varphi}^{\prime}(-m)\right| \leq c|z|\left(1+\log |z|^{-1}\right) \sup _{D_{\infty}}|\varphi|,
$$

and the assertion is proved.

4.1.2. Next we consider the behaviour near $z=1$ :

Proposition 4.2. There exists $c>0$ such that for all $\varphi \in \mathcal{O}^{1}(\overline{\mathbb{C}} \backslash[0,1])$ and $z \in D_{1}$ one has

$$
\begin{gathered}
\left|T \varphi(z)+z \varphi\left(\frac{1}{z}-1\right)-\sum_{m \geq 1} \varphi^{\prime}(-m)\right| \leq c|z-1| \sup _{D_{\infty}}|\varphi|, \\
\left|\sum_{\mathcal{M}} \varphi(z)-\varphi(z)+z \varphi\left(\frac{1}{z}-1\right)\right| \leq c|z-1|\left(1+\log |z-1|^{-1}\right) \sup _{D_{\infty}}|\varphi| .
\end{gathered}
$$

Proof. For $z \in D_{1}$ we have $\frac{1}{z}-1 \in D_{0}$. Moreover, there exists $c>0$ such that for $m \geq 2$ and $w$ on the segment with endpoints $1-m$ and $1 / z-m$ we have

$$
\left|\varphi^{\prime}(-w)\right| \leq c m^{-2} \sup _{D_{\infty}}|\varphi| .
$$

Since

$$
T \varphi(z)+z \varphi\left(\frac{1}{z}-1\right)-\sum_{m \geq 1} \varphi^{\prime}(-m)=z \sum_{m \geq 2}\left[\varphi(1-m)-\varphi\left(\frac{1}{z}-m\right)\right],
$$

from (4.5) one easily deduces (4.3).

Let $\tilde{\varphi}=\sum_{\mathcal{M}} \varphi$. We have $\tilde{\varphi}(z)=\varphi(z)+T \tilde{\varphi}(z)$, and

$$
\begin{aligned}
\tilde{\varphi}(z) & =\varphi(z)-z \varphi\left(\frac{1}{z}-1\right)+T \tilde{\varphi}(z)+z \tilde{\varphi}\left(\frac{1}{z}-1\right)-\sum_{m \geq 1} \tilde{\varphi}^{\prime}(-m) \\
& +z\left[\varphi\left(\frac{1}{z}-1\right)-\tilde{\varphi}\left(\frac{1}{z}-1\right)+\sum_{m \geq 1} \tilde{\varphi}^{\prime}(-m)\right]+(1-z) \sum_{m \geq 1} \tilde{\varphi}^{\prime}(-m) .
\end{aligned}
$$

By (4.3) one has

$$
\left|T \tilde{\varphi}(z)+z \tilde{\varphi}\left(\frac{1}{z}-1\right)-\sum_{m \geq 1} \tilde{\varphi}^{\prime}(-m)\right| \leq c|z-1| \sup _{D_{\infty}}|\tilde{\varphi}| .
$$

We also have $\sup _{D_{\infty}}|\tilde{\varphi}| \leq c \sup _{D_{\infty}}|\varphi|$ and by $(4.2)$

$$
\left|\varphi\left(\frac{1}{z}-1\right)-\tilde{\varphi}\left(\frac{1}{z}-1\right)+\sum_{m \geq 1} \tilde{\varphi}^{\prime}(-m)\right| \leq c|z-1|\left(1+\log |z-1|^{-1}\right) \sup _{D_{\infty}}|\varphi| .
$$

As $\left|\sum_{m \geq 1} \tilde{\varphi}^{\prime}(-m)\right| \leq c \sup _{D_{\infty}}|\tilde{\varphi}| \leq c \sup _{D_{\infty}}|\varphi|$, we get the second inequality.

Remark 4.3. It is easy to check that the estimates in Proposition 4.2 are valid if $z$ is such that $z-1 \in \Delta$. 
4.2. Boundary behaviour and continued fraction. Let $k \geq 1$ and let $m_{1}, \ldots$, $m_{k}$ be integers $\geq 1$. We denote by $D\left(m_{1}, \ldots, m_{k}\right)$ the set of $z_{0} \in D$ for which the complex continued fraction is

$$
z_{i}^{-1}=m_{i+1}+z_{i+1}, \quad 0 \leq i<k,
$$

with $z_{i} \in D$ for $0 \leq i<k$ and $z_{k} \in \Delta$. In the following we set for $0<i \leq k$

$$
\varepsilon_{i}= \begin{cases}0 & \text { if } m_{i}=1 \\ 1 & \text { if } m_{i}>1\end{cases}
$$

Proposition 4.4. For $\varphi \in \mathcal{O}^{1}(\overline{\mathbb{C}} \backslash[0,1])$ we have in $D\left(m_{1}, \ldots, m_{k}\right)$

$$
\begin{aligned}
T^{k} \varphi\left(z_{0}\right) & =\left(p_{k-1}-q_{k-1} z_{0}\right)\left[\varphi\left(z_{k}\right)+\varphi\left(z_{k}-1\right)+\varepsilon_{k} \varphi\left(z_{k}+1\right)\right] \\
& -\left(p_{k-2}-q_{k-2} z_{0}\right)\left(1+z_{k-1}\right) \varepsilon_{k-1} \varphi\left(-\frac{z_{k-1}}{1+z_{k-1}}\right)+R^{[k]}(\varphi)\left(z_{0}\right) .
\end{aligned}
$$

The remainder term $R^{[k]}(\varphi)$ is holomorphic in int $D\left(m_{1}, \ldots, m_{k}\right)$, continuous in $D\left(m_{1}, \ldots, m_{k}\right)$ and satisfies there

$$
\left|R^{[k]}(\varphi)\left(z_{0}\right)\right| \leq c k\left(\frac{\sqrt{5}+1}{2}\right)^{-k} \sup _{D_{\infty}}|\varphi| .
$$

Proof. One has

$$
T \varphi\left(z_{0}\right)=-z_{0}\left[\varphi\left(z_{1}\right)+\varphi\left(z_{1}-1\right)+\varepsilon_{1} \varphi\left(z_{1}+1\right)\right]+R^{\left(m_{1}\right)}(\varphi)\left(z_{0}\right),
$$

with

$$
\begin{aligned}
R^{\left(m_{1}\right)}(\varphi)\left(z_{0}\right) & =\sum_{m \geq 1} \varphi^{\prime}(-m)-z_{0} \sum_{m \geq 1,\left|m-m_{1}\right|>1}\left[\varphi\left(z_{1}+m_{1}-m\right)-\varphi(-m)\right] \\
& +z_{0} \sum_{m \geq 1,\left|m-m_{1}\right| \leq 1} \varphi(-m) .
\end{aligned}
$$

For $z_{0} \in D\left(m_{1}\right)$, one easily checks that $\left|R^{\left(m_{1}\right)}(\varphi)\left(z_{0}\right)\right| \leq c \sup _{D_{\infty}}|\varphi|$ and that $R^{\left(m_{1}\right)}(\varphi)$ is holomorphic in the neighborhood of $D\left(m_{1}\right)$.

Iterating (4.8) $k$ times we have

$$
\begin{aligned}
T^{k} \varphi\left(z_{0}\right) & =\prod_{i=0}^{k-1}\left(-z_{i}\right)\left[\varphi\left(z_{k}\right)+\varphi\left(z_{k}-1\right)+\varepsilon_{k} \varphi\left(z_{k}+1\right)\right] \\
& +\sum_{j=1}^{k-1}\left[\prod_{i=0}^{j-1}\left(-z_{i}\right)\right]\left(T^{k-j} \varphi\left(z_{j}-1\right)+\varepsilon_{j} T^{k-j} \varphi\left(z_{j}+1\right)\right) \\
& +\sum_{j=1}^{k}\left[\prod_{i=0}^{j-2}\left(-z_{i}\right)\right] R^{\left(m_{j}\right)}\left(T^{k-j} \varphi\right)\left(z_{j-1}\right) .
\end{aligned}
$$

We have here $\prod_{0}^{l-1}\left(-z_{i}\right)=p_{l-1}-q_{l-1} z_{0}$ and $\left|p_{l-1}-q_{l-1} z_{0}\right| \leq c q_{l}^{-1}$. The function

$$
R_{0}^{[k]}\left(z_{0}\right)=\sum_{j=1}^{k}\left[\prod_{i=0}^{j-2}\left(-z_{i}\right)\right] R^{\left(m_{j}\right)}\left(T^{k-j} \varphi\right)\left(z_{j-1}\right)
$$


is holomorphic in a neighborhood of $D\left(m_{1}, \ldots, m_{k}\right)$ and satisfies there

$$
\left|R_{0}^{[k]}\left(z_{0}\right)\right| \leq c \sum_{j=1}^{k} q_{j-1}^{-1} \sup _{D_{\infty}}\left|T^{k-j} \varphi\right| \leq c k\left(\frac{\sqrt{5}+1}{2}\right)^{-k} \sup _{D_{\infty}}|\varphi| .
$$

The function

$$
R_{1}^{[k]}\left(z_{0}\right)=\sum_{j=1}^{k-1}\left(p_{j-1}-q_{j-1} z_{0}\right) T^{k-j} \varphi\left(z_{j}-1\right)
$$

is holomorphic in the interior of $D\left(m_{1}, \ldots, m_{k}\right)$ (and even in a neighborhood of $D\left(m_{1}, \ldots, m_{k}\right)$ if $\left.m_{k}>1\right)$. Applying Proposition $4.1(k-j)$ times to each term of the sum we see that it is continuous in $D\left(m_{1}, \ldots, m_{k}\right)$ and satisfies there

$$
\left|R_{1}^{[k]}\left(z_{0}\right)\right| \leq c \sum_{j=1}^{k-1} q_{j}^{-1} \sup _{D_{\infty}}\left|T^{k-j-1} \varphi\right| \leq c k\left(\frac{\sqrt{5}+1}{2}\right)^{-k} \sup _{D_{\infty}}|\varphi| .
$$

The function

$$
R_{2}^{[k]}\left(z_{0}\right)=\sum_{j=1}^{k-2}\left(p_{j-1}-q_{j-1} z_{0}\right) \varepsilon_{j} T^{k-j} \varphi\left(z_{j}+1\right)
$$

is holomorphic in a neighborhood of $D\left(m_{1}, \ldots, m_{k}\right)$ and satisfies, according to Propositions 4.1 and 4.2 ,

$$
\left|R_{2}^{[k]}\left(z_{0}\right)\right| \leq c \sum_{j=1}^{k-2} q_{j}^{-1} \sup _{D_{\infty}}\left|T^{k-j-2} \varphi\right| \leq c k\left(\frac{\sqrt{5}+1}{2}\right)^{-k} \sup _{D_{\infty}}|\varphi| .
$$

Finally, we apply Proposition 4.2 to $\left(p_{k-2}-q_{k-2} z_{0}\right) \varepsilon_{k-1} T \varphi\left(z_{k-1}+1\right)$ and by summing up the different contributions to (4.9) we get the desired result.

\section{3. $H^{p}-$ estimates.}

4.3.1. Let $1<p<+\infty$. We consider the space $H^{p}\left(\mathbb{H}^{ \pm}\right)$of functions $F \in$ $\mathcal{O}^{1}(\overline{\mathbb{C}} \backslash[0,1])$ whose restrictions to both $\mathbb{H}^{+}$and $\mathbb{H}^{-}$belong to the Hardy space $H^{p}$ of these half-spaces, endowed with the norm

$$
\|F\|_{H^{p}\left(\mathbb{H}^{ \pm}\right)}=\left\|\left.F\right|_{\mathbb{H}^{+}}\right\|_{H^{p}}+\left\|\left.F\right|_{\mathbb{H}^{-}}\right\|_{H^{p}} .
$$

See references [Du, St] for details. Then, it is a classical result Ga that $F \in$ $H^{p}\left(\mathbb{H}^{ \pm}\right)$if and only if the associated hyperfunction $u$ belongs to $L^{p}([0,1])$ and that the correspondence is an isomorphism of Banach spaces.

But we know [MMY] that $T$ acting on $L^{p}([0,1])$ has spectral radius $\leq \frac{\sqrt{5}-1}{2}$. Consequently the same is true for $T$ acting on $H^{p}\left(\mathbb{H}^{ \pm}\right)$.

4.3.2. For the case $p=\infty$ and on the larger domain $\overline{\mathbb{C}} \backslash[0,1]$ we have the following

Proposition 4.5. The restriction of $T$ to $H^{\infty}(\overline{\mathbb{C}} \backslash[0,1]) \cap \mathcal{O}^{1}(\overline{\mathbb{C}} \backslash[0,1])$ is a bounded operator on this space with spectral radius $\leq \frac{\sqrt{5}-1}{2}$. 
Proof. Let $k \geq 1, \varphi \in H^{\infty}(\overline{\mathbb{C}} \backslash[0,1]) \cap \mathcal{O}^{1}(\overline{\mathbb{C}} \backslash[0,1])$ and $z_{0} \in \overline{\mathbb{C}} \backslash[0,1]$. We estimate $\left|T^{k} \varphi\left(z_{0}\right)\right|$ in various cases.

(i) If $z_{0} \in D_{\infty}$ we have

$$
\left|T^{k} \varphi\left(z_{0}\right)\right| \leq c\left(\frac{\sqrt{5}+1}{2}\right)^{-k} \sup _{D_{\infty}}|\varphi|,
$$

according to Remark 3.4.

(ii) If $z_{0} \in D_{0} \cup H_{0} \cup H_{0}^{\prime}$ we have, according to Proposition 4.1 and Remark 3.4,

$$
\left|T^{k} \varphi\left(z_{0}\right)\right| \leq c \sup _{D_{\infty}}\left|T^{k-1} \varphi\right| \leq c\left(\frac{\sqrt{5}+1}{2}\right)^{-k} \sup _{D_{\infty}}|\varphi| .
$$

(iii) If $z_{0} \in D_{1}$ we have, according to Proposition 4.2,

$$
\left|T^{k} \varphi\left(z_{0}\right)\right| \leq c\left[\sup _{D_{\infty}}\left|T^{k-1} \varphi\right|+\left|T^{k-1} \varphi\left(\frac{1}{z_{0}}-1\right)\right|\right]
$$

which gives, for $k=1,\left|T \varphi\left(z_{0}\right)\right| \leq c\|\varphi\|_{H^{\infty}}$, and for $k>1$, according to Proposition 4.1,

$$
\left|T^{k} \varphi\left(z_{0}\right)\right| \leq c \sup _{D_{\infty}}\left|T^{k-2} \varphi\right| \leq c\left(\frac{\sqrt{5}+1}{2}\right)^{-k} \sup _{D_{\infty}}|\varphi| .
$$

(iv) If $z_{0} \in D$ has continued fraction $z_{i}^{-1}=m_{i+1}+z_{i+1}$ with $0 \leq i<l, z_{l} \in \Delta \backslash D$, $l<k$, we apply Proposition 4.4 to write

$$
\begin{aligned}
& T^{k} \varphi\left(z_{0}\right)=\left(p_{l-1}-q_{l-1} z_{0}\right)\left[T^{k-l} \varphi\left(z_{l}\right)+T^{k-l} \varphi\left(z_{l}-1\right)+\varepsilon_{l} T^{k-l} \varphi\left(z_{l}+1\right)\right] \\
& \quad-\left(p_{l-2}-q_{l-2} z_{0}\right)\left(1+z_{l-1}\right) \varepsilon_{l-1} T^{k-l} \varphi\left(\frac{-z_{l-1}}{1+z_{l-1}}\right)+R^{[l]}\left(T^{k-l} \varphi\right)\left(z_{0}\right) .
\end{aligned}
$$

Here, we have $z_{l}, z_{l}-1$ and $\frac{-z_{l-1}}{1+z_{l-1}}$ in $D_{\infty} \cup D_{0} \cup H_{0} \cup H_{0}^{\prime}$, hence the value of $T^{k-l} \varphi$ at these points is in absolute value less than $c \sup _{D_{\infty}}\left|T^{k-l-1} \varphi\right|$ (Proposition 4.1). We also have, according to Proposition 4.2 and Proposition 4.1

$$
\left|T^{k-l} \varphi\left(z_{l}+1\right)\right| \leq \begin{cases}c\|\varphi\|_{H^{\infty}} & \text { if } k=l+1 \\ c \sup _{D_{\infty}}\left|T^{k-l-2} \varphi\right| & \text { if } k>l+1\end{cases}
$$

On the other hand we have

$$
\begin{aligned}
& \left|p_{l-2}-q_{l-2} z_{0}\right| \leq c q_{l-1}^{-1} \leq c\left(\frac{\sqrt{5}+1}{2}\right)^{-l} \\
& \left|p_{l-1}-q_{l-1} z_{0}\right| \leq c q_{l}^{-1} \leq c\left(\frac{\sqrt{5}+1}{2}\right)^{-l}
\end{aligned}
$$

and thus from the estimate of $R^{[l]}$ in Proposition 4.4 and from Remark 3.4 we have

$$
\left|T^{k} \varphi\left(z_{0}\right)\right| \leq \begin{cases}c l\left(\frac{\sqrt{5}+1}{2}\right)^{-l}\|\varphi\|_{H^{\infty}} & \text { if } k=l+1 \\ c k\left(\frac{\sqrt{5}+1}{2}\right)^{-k} \sup _{D_{\infty}}|\varphi| & \text { if } k>l+1\end{cases}
$$


(v) If $z_{0} \in D$ has continued fraction $z_{i}^{-1}=m_{i+1}+z_{i+1}$ with $0 \leq i<k, z_{k} \in \Delta$, we again apply Proposition 4.4 to get

$$
\begin{aligned}
T^{k} \varphi\left(z_{0}\right) & =\left(p_{k-1}-q_{k-1} z_{0}\right)\left[\varphi\left(z_{k}\right)+\varphi\left(z_{k}-1\right)+\varepsilon_{k} \varphi\left(z_{k}+1\right)\right] \\
& -\left(p_{k-2}-q_{k-2} z_{0}\right)\left(1+z_{k-1}\right) \varepsilon_{k-1} \varphi\left(-\frac{z_{k-1}}{1+z_{k-1}}\right)+R^{[k]}(\varphi)\left(z_{0}\right),
\end{aligned}
$$

which gives

$$
\left|T^{k} \varphi\left(z_{0}\right)\right| \leq c q_{k-1}^{-1}\|\varphi\|_{H^{\infty}}+c k\left(\frac{\sqrt{5}+1}{2}\right)^{-k} \sup _{D_{\infty}}|\varphi| .
$$

By collecting the estimates obtained in (i)-(v) we get the desired result.

4.3.3. Let us now consider the conformally invariant version of $H^{p}, 1<p<+\infty$. The application $w \mapsto z=\frac{(w+1)^{2}}{4 w}$ is a conformal representation of $\mathbb{D}$ on $\mathbb{C} \backslash[0,1]$; letting $w=e^{2 \pi i \theta}$, one finds $z=\cos ^{2} \theta$ and thus $|d z|=2|\sin \theta||\cos \theta| d \theta$, i.e. $d \theta=$ $\frac{d x}{2 \sqrt{x(1-x)}}$. We can therefore identify $L^{p}\left(\mathbb{T}^{1}\right)$ with the direct sum of two copies of $L^{p}\left([0,1], \frac{d x}{2 \sqrt{x(1-x)}}\right)$ (one for each side of $[0,1]$ ). Now let $\varphi \in \mathcal{O}^{1}(\overline{\mathbb{C}} \backslash[0,1]) \cap$ $H^{p}(\overline{\mathbb{C}} \backslash[0,1])$, which we will assume to be real. The associated hyperfunction $u$ is $u=\mathfrak{I} m \varphi(x+i 0)$, and $u \in L^{p}\left([0,1], \frac{d x}{2 \sqrt{x(1-x)}}\right)$. We have the following

Proposition 4.6. For $p \geq 1$ the operator $T$ defines a bounded operator on the space $L^{p}\left([0,1], \frac{d x}{2 \sqrt{x(1-x)}}\right)$ and its spectral radius is $\leq \frac{\sqrt{5}-1}{2}$.

Proof. One has $T u(x)=x u\left(\frac{1}{x}-m\right)$ if $\frac{1}{m+1} \leq x \leq \frac{1}{m}$, thus

$$
\begin{aligned}
\int_{1 /(m+1)}^{1 / m}|T u(x)|^{p} \frac{d x}{2 \sqrt{x(1-x)}} & \leq m^{1 / 2-p} \int_{1 /(m+1)}^{1 / m}\left|u\left(\frac{1}{x}-m\right)\right|^{p} \frac{d x}{2 \sqrt{(1-x)}} \\
& \leq m^{1 / 2-p} \int_{0}^{1}|u(s)|^{p}\left(1-\frac{1}{s+m}\right)^{-1 / 2} \frac{d s}{(s+m)^{2}} \\
& \leq m^{1 / 2-p} \int_{0}^{1}|u(s)|^{p}(s+m)^{-3 / 2} \frac{d s}{\sqrt{s}}
\end{aligned}
$$

Summing over $m \geq 1$ we have

$$
\|T u\|_{L^{p}([0,1], d x / 2 \sqrt{x(1-x)})} \leq\left[\sum_{m \geq 1} m^{-p-1}\right]^{1 / p}\|u\|_{L^{p}([0,1], d x / \sqrt{x})},
$$

which proves the first assertion.

As far as the spectral radius is concerned, let us consider an integer $k>1$ and the set $\mathcal{I}(k)$ of the intervals of definition of the branches of $A^{k}$. For $I \in \mathcal{I}(k)$ one has (we denote by $x_{I}$ the center of $I$ )

$$
\begin{aligned}
\int_{I}\left|T^{k} u(x)\right|^{p} \frac{d x}{2 \sqrt{x(1-x)}} & =\int_{I} \beta_{k-1}(x)^{p}\left|u\left(A^{k} x\right)\right|^{p} \frac{d x}{2 \sqrt{x(1-x)}} \\
& \leq c|I|^{p}\left[x_{I}\left(1-x_{I}\right)\right]^{-1 / 2} \int_{I}\left|u\left(A^{k} x\right)\right|^{p} d x
\end{aligned}
$$


since $c^{-1}\left[x_{I}\left(1-x_{I}\right)\right]^{-1 / 2} \leq[x(1-x)]^{-1 / 2} \leq c\left[x_{I}\left(1-x_{I}\right)\right]^{-1 / 2}$ for all $x \in I, I \in$ $\mathcal{I}(k)$ and $k>1$; also $c^{-1}|I| \leq \beta_{k-1}(x) \leq c|I|$ for all $x \in I$. From this we get

$$
\int_{I}\left|T^{k} u(x)\right|^{p} \frac{d x}{2 \sqrt{x(1-x)}} \leq c|I|^{p+1}\left[x_{I}\left(1-x_{I}\right)\right]^{-1 / 2}\|u\|_{L^{p}([0,1])}^{p}
$$

(since $A^{k}$ has bounded distortion on $I$ ).

Taking the sum over all the intervals $I$ (i.e. the branches of $A^{k}$ ), since the $I \in \mathcal{I}(k)$ form a partition $(\bmod 0)$ of $[0,1]$, one obtains

$$
\left\|T^{k} u\right\|_{L^{p}\left([0,1], \frac{d x}{2 \sqrt{x(1-x)}}\right)}^{p} \leq c \int_{0}^{1} \frac{d x}{\sqrt{x(1-x)}}\|u\|_{L^{p}([0,1])}^{p}\left[\max _{\mathcal{I}(k)}|I|\right]^{p},
$$

which proves the second part of the proposition.

Corollary 4.7. For $p>1$, the operator $T$ maps $\mathcal{O}^{1}(\overline{\mathbb{C}} \backslash[0,1]) \cap H^{p}(\overline{\mathbb{C}} \backslash[0,1])$ into itself, is bounded and its spectral radius is $\leq \frac{\sqrt{5}-1}{2}$.

Proof. Let $\varphi \in \mathcal{O}^{1}(\overline{\mathbb{C}} \backslash[0,1]) \cap H^{p}(\overline{\mathbb{C}} \backslash[0,1])$ be real, and let $u=\Im m \varphi(x+i 0)$ be the associated hyperfunction. For $k \geq 0$ the function of $\mathcal{O}^{1}(\overline{\mathbb{C}} \backslash[0,1])$ associated to $T^{k} u$ is $T^{k} \varphi$. Using the conformal representation of $\overline{\mathbb{C}} \backslash[0,1]$ onto $\mathbb{D}$ and the fact that the Hilbert transform is bounded on $L^{p}(d \theta)$ one obtains the desired result.

4.3.4. Here we consider the operator $\sum_{\mathcal{M}}$ acting on the space $H^{1}(\overline{\mathbb{C}} \backslash[0,1]) \cap$ $\mathcal{O}^{1}(\overline{\mathbb{C}} \backslash[0,1])$.

Lemma 4.8. For all $g \in \mathcal{M}$ the restriction to $H^{1}(\overline{\mathbb{C}} \backslash[0,1]) \cap \mathcal{O}^{1}(\overline{\mathbb{C}} \backslash[0,1])$ of $L_{g}$ is a bounded operator of this space into itself.

Proof. It is sufficient to consider the case $g=g(m), m \geq 1$. One then has

$$
L_{g(m)}=\sigma \circ \tau^{-1} \circ \chi_{1} \circ \chi_{0} \circ \tau \circ \sigma \circ \iota_{m} \circ \tau_{m},
$$

where

$\tau_{m}$ is the isomorphism $\varphi(z) \mapsto \varphi\left(\frac{1}{z}-m\right)$ of $H^{1}(\overline{\mathbb{C}} \backslash[0,1])$ onto

$$
H^{1}(\overline{\mathbb{C}} \backslash[1 /(m+1), 1 / m]) ;
$$

$\iota_{m}$ is the canonical injection of $H^{1}(\overline{\mathbb{C}} \backslash[1 /(m+1), 1 / m])$ into $H^{1}(\overline{\mathbb{C}} \backslash[0,1])$;

$\sigma$ is the bounded operator $\varphi \mapsto \varphi-\varphi(\infty)$ of $H^{1}(\overline{\mathbb{C}} \backslash[0,1])$ into $H^{1}(\overline{\mathbb{C}} \backslash[0,1]) \cap$ $\mathcal{O}^{1}(\overline{\mathbb{C}} \backslash[0,1])$

$\tau$ is the isomorphism $\varphi \mapsto \varphi_{1}(w)=\varphi\left(\frac{(w+1)^{2}}{4 w}\right)$ of $H^{1}(\overline{\mathbb{C}} \backslash[0,1])$ onto $H^{1}(\mathbb{D})$, whose restriction to $H^{1}(\overline{\mathbb{C}} \backslash[0,1]) \cap \mathcal{O}^{1}(\overline{\mathbb{C}} \backslash[0,1])$ is an isomorphism onto $\left\{\varphi_{1} \in H^{1}(\mathbb{D}), \varphi_{1}(0)=0\right\} ;$

$\chi_{0}$ is the isomorphism $\varphi_{1}(w) \mapsto \frac{1}{w} \varphi_{1}(w)$ of $\left\{\varphi_{1} \in H^{1}(\mathbb{D}), \varphi_{1}(0)=0\right\}$ onto $H^{1}(\mathbb{D})$;

$\chi_{1}$ is the multiplication operator by the function $-\frac{(w+1)^{2}}{4} \in H^{\infty}(\mathbb{D})$ into $H^{1}(\mathbb{D})$.

We now want to estimate the norm of $L_{g}$ acting on $H^{1}(\overline{\mathbb{C}} \backslash[0,1]) \cap \mathcal{O}^{1}(\overline{\mathbb{C}} \backslash[0,1])$.

Proposition 4.9. There exists a constant $K>0$ such that, if $g \in \mathcal{M}$ is different from $\left(\begin{array}{ll}1 & 0 \\ 0 & 1\end{array}\right),\left(\begin{array}{ll}0 & 1 \\ 1 & 1\end{array}\right)$ (thus $\left.d>b>0\right)$ one has

$$
\left\|L_{g}\right\|_{H^{1}} \leq K d^{-5 / 2}[\min (b, d-b)]^{-1 / 2} \log (1+d) .
$$


Proof. A function $\varphi \in H^{1}(\overline{\mathbb{C}} \backslash[0,1])$ has non-tangential limits at almost all points of the boundary and

$$
\|\varphi\|_{H^{1}}=\int_{0}^{1} \frac{|\varphi(x+i 0)| d x}{2 \sqrt{x(1-x)}}+\int_{0}^{1} \frac{|\varphi(x-i 0)| d x}{2 \sqrt{x(1-x)}} .
$$

On the other hand a function $\varphi \in H^{1}(\overline{\mathbb{C}} \backslash[0,1])$ verifies

$$
\begin{aligned}
& |\varphi(t)| \leq C|t|^{-1 / 2}\|\varphi\|_{H^{1}} \quad \forall t \in(-1,0), \\
& |\varphi(t)| \leq C(t-1)^{-1 / 2}\|\varphi\|_{H^{1}} \quad \forall t \in(1,2),
\end{aligned}
$$

as one can check directly by applying Poisson's integral formula. Moreover, if it belongs to $H^{1}(\overline{\mathbb{C}} \backslash[0,1]) \cap \mathcal{O}^{1}(\overline{\mathbb{C}} \backslash[0,1])$ the same argument leads to the estimates

$$
|\varphi(z)| \leq C|z|^{-1}\|\varphi\|_{H^{1}}, \quad\left|\varphi^{\prime}(z)\right| \leq C|z|^{-2}\|\varphi\|_{H^{1}}, \quad\left|\varphi^{\prime \prime}(z)\right| \leq C|z|^{-3}\|\varphi\|_{H^{1}},
$$

for all $z$ such that $|z-1 / 2|>1$.

Given these preliminary elementary estimates, let $\varphi \in H^{1}(\overline{\mathbb{C}} \backslash[0,1]) \cap$ $\mathcal{O}^{1}(\overline{\mathbb{C}} \backslash[0,1]), g=\left(\begin{array}{ll}a & b \\ c & d\end{array}\right) \in \mathcal{M}$, with $d>b>0$. Let $\tilde{\varphi}=L_{g} \varphi ; \tilde{\varphi}$ is holomorphic outside the interval with end points $b / d$ and $(a+b) /(c+d)$. We must estimate $\int_{0}^{1} \frac{|\tilde{\varphi}(x \pm i 0)| d x}{\sqrt{x(1-x)}}$ and by symmetry it is enough to consider $\tilde{\varphi}(x+i 0)$. We define an interval $I_{g}$ of the following form:

(i) if $g=\left(\begin{array}{cc}0 & 1 \\ 1 & m\end{array}\right), m \geq 2, I_{g}=[0,3 / 2 m]$;

(ii) if $g=\left(\begin{array}{cc}1 & m-1 \\ 1 & m\end{array}\right), m \geq 2, I_{g}=[1-3 / 2 m, 1]$;

(iii) in all the other cases one has $c>a>0, \frac{a}{c}=\frac{b}{d}+\frac{\varepsilon_{g}}{c d} ; I_{g}$ is the interval with end points $\frac{b}{d}+\frac{3 \varepsilon_{g}}{2 c d}$ and $\frac{b}{d}-\frac{\varepsilon_{g}}{2 c d}$; clearly $I_{g} \subset[0,1]$.

We will now directly estimate the integral of $\frac{\tilde{\varphi}(x+i 0) d x}{\sqrt{x(1-x)}}$ on $I_{g}$ and on $[0,1] \backslash I_{g}$. We begin with the latter.

If $x \in[0,1] \backslash I_{g}, \tilde{\varphi}$ is holomorphic in a neighborhood of $x$ and one has

$$
\tilde{\varphi}(x)=c^{-2}(a-c x)^{-1} \int_{0}^{1}(1-t) \varphi^{\prime \prime}\left(-\frac{d}{c}+\varepsilon_{g} \frac{t}{c(a-c x)}\right) d t .
$$

We have

$$
x \notin I_{g} \Rightarrow \frac{\varepsilon_{g}}{c(a-c x)} \in\left[-2 \frac{d}{c}, \frac{2}{3} \frac{d}{c}\right],
$$

thus the values of the second derivative in the integral are $\leq C c^{3} d^{-3}\|\varphi\|_{H^{1}}$, by the third estimate of (4.15), and one has

$$
|\tilde{\varphi}(x)| \leq C d^{-3}|x-b / d|^{-1}\|\varphi\|_{H^{1}},
$$

since $x-a / c$ and $x-b / d$ are comparable outside $I_{g}$. We thus obtain

$$
\int_{[0,1] \backslash I_{g}} \frac{|\tilde{\varphi}(x \pm i 0)| d x}{\sqrt{x(1-x)}} \leq C d^{-3}\|\varphi\|_{H^{1}} \int_{[0,1] \backslash I_{g}} \frac{d x}{\sqrt{x(1-x)}\left|x-\frac{b}{d}\right|} .
$$


An easy estimate of this last integral gives

$$
\int_{[0,1] \backslash I_{g}} \frac{|\tilde{\varphi}(x \pm i 0)| d x}{\sqrt{x(1-x)}} \leq C d^{-5 / 2}[\min (b, d-b)]^{-1 / 2} \log (1+\min (b, d-b)) .
$$

For the integral inside $I_{g}$ we distinguish the three different contributions to $\tilde{\varphi}=$ $L(g) \varphi$. First of all one has (applying the second estimate of (4.15))

$$
\left|c^{-1} \varphi^{\prime}(-d / c)\right| \leq C c d^{-2}\|\varphi\|_{H^{1}}
$$

and

$$
\int_{I_{g}} \frac{d x}{\sqrt{x(1-x)}} \leq C c^{-1} d^{-1 / 2}[\min (b, d-b)]^{-1 / 2}\|\varphi\|_{H^{1}}
$$

thus

$$
\int_{I_{g}} \frac{\left|c^{-1} \varphi^{\prime}(-d / c)\right| d x}{\sqrt{x(1-x)}} \leq C d^{-5 / 2}[\min (b, d-b)]^{-1 / 2}\|\varphi\|_{H^{1}} .
$$

For $x \in I_{g}$, by applying (4.15) and (4.16) one has

$$
|(a-c x) \varphi(-d / c)| \leq C c d^{-2}\|\varphi\|_{H^{1}},
$$

from which follows the same estimate above for the second term. We are left with

$$
I=\int_{I_{g}} \frac{|a-c x \| \varphi(y \pm i 0)|}{\sqrt{x(1-x)}} d x, y=\frac{d x-b}{a-c x} .
$$

Inside $I_{g}$ one has $\frac{|a-c x|}{\sqrt{x(1-x)}} \leq C d^{-1 / 2}[\min (b, d-b)]^{-1 / 2} ;$ on the other hand one has $\int_{I_{g}}|\varphi(y \pm i 0)| d x=c^{-2} \int \frac{|\varphi(y \pm i 0)| d y}{(y+d / c)^{2}}$, where this integral is taken on the complement in $\overline{\mathbb{R}}$ of the interval $\left[-\frac{3 d}{c},-\frac{d}{3 c}\right]$ (if $c>1$; when $g=\left(\begin{array}{cc}0 & 1 \\ 1 & m\end{array}\right)$ or $\left(\begin{array}{cc}1 & m-1 \\ 1 & m\end{array}\right), m \geq 2$, the integral is taken from $-m / 3$ to $\left.+\infty\right)$.

One then has

$$
\begin{aligned}
& \int_{-1 / 2}^{3 / 2} \frac{|\varphi(y \pm i 0)| d y}{(y+d / c)^{2}} \leq C c^{2} d^{-2}\|\varphi\|_{H^{1}}, \\
& \int_{-d / 3 c}^{-1 / 2} \frac{|\varphi(y \pm i 0)| d y}{(y+d / c)^{2}} \leq C c^{2} d^{-2}\|\varphi\|_{H^{1}} \log \left(1+\frac{d}{c}\right),
\end{aligned}
$$

and the same for $\int_{3 / 2}^{3 d / c}$. Finally one has

$$
\int_{|y|>3 d / c} \frac{|\varphi(y \pm i 0)| d y}{(y+d / c)^{2}} \leq C c^{2} d^{-2}\|\varphi\|_{H^{1}} .
$$

Putting the various bounds together one gets

$$
\int_{I_{g}} \frac{|\tilde{\varphi}(x \pm i 0)| d x}{\sqrt{x(1-x)}} \leq C d^{-5 / 2}[\min (b, d-b)]^{-1 / 2} \log (1+d / c)\|\varphi\|_{H^{1}} .
$$

To conclude the proof it is now enough to note that $\log (1+d / c) \leq \log (1+d)$ and $\log (1+c \min (b, d-b)) \leq \log \left(1+d^{2}\right) \leq 2 \log (1+d)$. 
Corollary 4.10. The series $\sum_{\mathcal{M}}\left\|L_{g}\right\|_{H^{1}}$ is convergent. $\sum_{\mathcal{M}}$ defines a bounded operator of $H^{1}(\overline{\mathbb{C}} \backslash[0,1]) \cap \mathcal{O}^{1}(\overline{\mathbb{C}} \backslash[0,1])$ into itself; moreover $T$ is a contraction of this space, its spectral radius being bounded above by $\frac{\sqrt{5}-1}{2}$.

Proof. Let $k \geq 1$. We will take the sum of $\left\|L_{g}\right\|_{H^{1}}$ on the elements $g \in \mathcal{M}$ which are the product of exactly $k$ generators $g(m)$. The branch of $A^{k}$ associated to $g$ has domain $\left[\frac{a+b}{c+d}, \frac{b}{d}\right]=I(g)$ and on this interval the max and the min of $x(1-x)$ are comparable. The length $[d(c+d)]^{-1}$ of $I(g)$ is bounded below by $\frac{1}{2} d^{-2}$, thus

$$
\left\|L_{g}\right\|_{H^{1}} \leq \frac{K d^{-3} \log (1+d)}{\sqrt{\frac{b}{d}\left(1-\frac{b}{d}\right)}} \leq K^{\prime} d^{-1} \log (1+d) \int_{I(g)} \frac{d x}{\sqrt{x(1-x)}} .
$$

When we sum on the elements $g$ considered, the intervals $I(g)$ form a partition of $[0,1] \bmod 0$, thus we get

$$
\sum_{m_{1}, \ldots, m_{k}, g=g\left(m_{1}\right) \cdots g\left(m_{k}\right)}\left\|L_{g}\right\|_{H^{1}} \leq K^{\prime \prime} \max _{m_{1}, \ldots, m_{k}}\left[d^{-1} \log (1+d)\right],
$$

which gives the desired result.

4.4. Real holomorphic functions with bounded real part. We denote by $E$ the space of functions $\varphi \in \mathcal{O}^{1}(\overline{\mathbb{C}} \backslash[0,1])$ whose real part is bounded and endowed with the norm $\|\varphi\|_{E}=\sup _{\overline{\mathbb{C}} \backslash[0,1]}|\mathfrak{R} e \varphi|$. We then have for $|\mathfrak{I} m z| \leq 1 / 2$

$$
|\Im m \varphi(z)| \leq \frac{2}{\pi} \log [(2 \sqrt{2}-2)|\Im m z|]^{-1}\|\varphi\|_{E},
$$

as one can prove from the analogous estimate for functions in the unit disk $\mathbb{D}$

$$
|\Im m \Phi(w)| \leq \frac{2}{\pi} \sup _{\vartheta \in[0,1]}\left|\mathfrak{R} e \Phi\left(e^{2 \pi i \vartheta}\right)\right| \log \frac{1+|w|}{1-|w|},
$$

applying the conformal representation of $\overline{\mathbb{C}} \backslash[0,1] \rightarrow \mathbb{D}, w=(\sqrt{z}-\sqrt{z-1})^{2}$.

Proposition 4.11. The restriction of $T$ to $E$ is a bounded operator with spectral radius $\leq \frac{\sqrt{5}-1}{2}$.

Proof. Let $\varphi \in E, k \geq 1, z_{0} \in \overline{\mathbb{C}} \backslash[0,1]$. We estimate $\mathfrak{R} e T^{k} \varphi\left(z_{0}\right)$ in various cases.

(i) If $z_{0} \in D_{\infty}$ we have

$$
\left|\Re e T^{k} \varphi\left(z_{0}\right)\right| \leq\left|T^{k} \varphi\left(z_{0}\right)\right| \leq c\left(\frac{\sqrt{5}+1}{2}\right)^{-k} \sup _{D_{\infty}}|\varphi|,
$$

and, on the other hand, for all $\varphi \in E, \sup _{D_{\infty}}|\varphi| \leq C\|\varphi\|_{E}$.

(ii) If $z_{0} \in D_{0} \cup H_{0} \cup H_{0}^{\prime}$, or if $z_{0} \in D_{1}, k>1$, or if $z_{0} \in D$ has continued fraction $z_{i}^{-1}=m_{i+1}+z_{i+1}, 0 \leq i<l$, with $z_{l} \in \Delta \backslash D, l<k-1$, we have obtained in the proof of Proposition 4.5 the estimate

$$
\left|T^{k} \varphi\left(z_{0}\right)\right| \leq c k\left(\frac{\sqrt{5}+1}{2}\right)^{-k} \sup _{D_{\infty}}|\varphi| .
$$

(iii) If $z_{0} \in D_{1}, k=1$, we have from Proposition 4.2

$$
\left|T \varphi\left(z_{0}\right)+z_{0} \varphi\left(\frac{1}{z_{0}}-1\right)\right| \leq c \sup _{D_{\infty}}|\varphi|,
$$


moreover

$$
\left|\mathfrak{R} e z_{0} \mathfrak{R} e \varphi\left(\frac{1}{z_{0}}-1\right)\right| \leq C\|\varphi\|_{E}
$$

and

$$
\left|\mathfrak{I} m\left(\frac{1}{z_{0}}-1\right)\right| \geq C^{-1}\left|\Im m z_{0}\right|
$$

hence from $(*)$

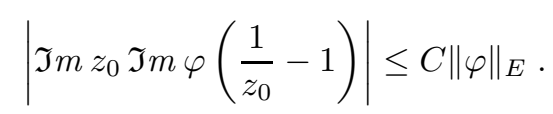

(iv) If $z_{0} \in D$ has continued fraction $z_{i}^{-1}=m_{i+1}+z_{i+1}, 0 \leq i<k-1$, with $z_{k-1} \in \Delta \backslash D$, the only term in the proof of Proposition 4.5 which gives some trouble is $\left(p_{k-2}-q_{k-2} z_{0}\right) \varepsilon_{k-2} T \varphi\left(z_{k-1}+1\right)$ (the others are once again dominated by $\left.C k\left(\frac{\sqrt{5}+1}{2}\right)^{-k} \sup _{D_{\infty}}|\varphi|\right)$. In fact, from Proposition 4.2 , we are even left with

$$
\left(p_{k-2}-q_{k-2} z_{0}\right) \varepsilon_{k-2}\left(1+z_{k-1}\right) \varphi\left(\frac{1}{1+z_{k-1}}-1\right) .
$$

We have here $\left(p_{k-2}-q_{k-2} z_{0}\right)\left(1+z_{k-1}\right)=\left(p_{k-2}-q_{k-2} z_{0}\right)-\left(p_{k-1}-q_{k-1} z_{0}\right)$; hence

$$
\begin{aligned}
& \left|\mathfrak{R} e\left[\left(p_{k-2}-q_{k-2} z_{0}\right)\left(1+z_{k-1}\right)\right]\right| \leq C q_{k-1}^{-1} \\
& \left|\mathfrak{I} m\left[\left(p_{k-2}-q_{k-2} z_{0}\right)\left(1+z_{k-1}\right)\right]\right| \leq C q_{k-1}\left|\mathfrak{I} m z_{0}\right| \leq C q_{k-1}^{-1}\left|\mathfrak{I} m z_{k-1}\right|
\end{aligned}
$$

and

$$
\left|\Im m\left(\frac{1}{1+z_{k-1}}-1\right)\right| \geq C^{-1}\left|\mathfrak{I} m z_{k-1}\right| .
$$

Thus, from $(*)$ we get

$$
\left|\Re e\left(p_{k-2}-q_{k-2} z_{0}\right)\left(1+z_{k-1}\right) \varphi\left(\frac{1}{1+z_{k-1}}-1\right)\right| \leq C q_{k-1}^{-1}\|\varphi\|_{E}
$$

and finally $\left|\mathfrak{R} e T^{k} \varphi\left(z_{0}\right)\right| \leq C k\left(\frac{\sqrt{5}+1}{2}\right)^{-k}\|\varphi\|_{E}$.

(v) If $z_{0} \in D$ has continued fraction $z_{i}^{-1}=m_{i+1}+z_{i+1}, 0 \leq i<k$, with $z_{k} \in \Delta$, we apply Proposition 4.4. We have

$$
\begin{aligned}
& \left|\mathfrak{R} e\left(p_{k-1}-q_{k-1} z_{0}\right)\right| \leq C q_{k}^{-1}, \\
& \left|\Im m\left(p_{k-1}-q_{k-1} z_{0}\right)\right| \leq q_{k-1}\left|\Im m z_{0}\right| \leq C q_{k-1} q_{k}^{-2}\left|\Im m z_{k}\right| ;
\end{aligned}
$$

hence, for $\varepsilon=-1,0,1$, from $(*)$

$$
\left|\mathfrak{R} e\left[\left(p_{k-1}-q_{k-1} z_{0}\right) \varphi\left(z_{k}+\varepsilon\right)\right]\right| \leq C q_{k}^{-1}\|\varphi\|_{E} .
$$

We deal similarly with

$$
\mathfrak{R} e\left[\left(p_{k-2}-q_{k-2} z_{0}\right)\left(1+z_{k-1}\right) \varphi\left(-\frac{z_{k-1}}{1+z_{k-1}}\right)\right]
$$


(see (iv) above) and conclude that

$$
\left|\mathfrak{R} e T^{k} \varphi\left(z_{0}\right)\right| \leq C k\left(\frac{\sqrt{5}+1}{2}\right)^{-k}\|\varphi\|_{E} .
$$

\section{The Complex Brunno function}

In this section we introduce and study the complex Brjuno function. Preliminarily we need some further results on the monoid $\mathcal{M}$ and on the algebraic properties of $\sum_{\mathcal{M}}$ and $\sum_{Z \mathcal{M}}$.

\subsubsection{Let us recall that one has}

$$
Z \mathcal{M}=Z \mathcal{M}\left(\begin{array}{ll}
1 & 1 \\
0 & 1
\end{array}\right) \sqcup Z \mathcal{M}\left(\begin{array}{ll}
0 & 1 \\
1 & 1
\end{array}\right) .
$$

More precisely, if one denotes $\mathcal{M}^{*}=\mathcal{M} \backslash\{1\}$, one has

$$
\mathcal{M}\left(\begin{array}{ll}
1 & 1 \\
0 & 1
\end{array}\right) \sqcup \mathcal{M}\left(\begin{array}{ll}
0 & 1 \\
1 & 1
\end{array}\right)=\mathcal{M}^{*} \sqcup\left\{\left(\begin{array}{ll}
1 & 1 \\
0 & 1
\end{array}\right)\right\} .
$$

5.0.2. Let $\varphi \in \mathcal{O}^{1}(\overline{\mathbb{C}} \backslash[0,1])$. Let

$$
\varphi_{1}=\left(L\left(\begin{array}{ll}
0 & 1 \\
1 & 1
\end{array}\right)+L\left(\begin{array}{ll}
1 & 1 \\
0 & 1
\end{array}\right)\right) \varphi \in \mathcal{O}^{1}(\overline{\mathbb{C}} \backslash[1 / 2,2]) .
$$

The family $\left(L_{g} \varphi\right)_{g \in \mathcal{M}}$ is uniformly summable on compact subsets of $\overline{\mathbb{C}} \backslash[0,1]$, thus

$$
\sum_{\mathcal{M}} \varphi(z)-\varphi(z)=\sum_{\mathcal{M}} \varphi_{1}(z)-\varphi(z-1) .
$$

On the other hand $\sum_{\mathcal{M}} \varphi_{1}=\varphi_{1}+\sum_{\mathcal{M}}\left(T \varphi_{1}\right)$ and $T \varphi_{1} \in \mathcal{O}^{1}(\overline{\mathbb{C}} \backslash[0,1])$. We can therefore conclude that

$$
\sum_{Z \mathcal{M}} \varphi(z)=\sum_{Z}\left[\varphi_{1}+\sum_{\mathcal{M}}\left(T \varphi_{1}\right)\right]
$$

where the definition of $\sum_{Z} \varphi_{1}, \varphi_{1} \in \mathcal{O}^{1}(\overline{\mathbb{C}} \backslash[1 / 2,2])$, is obtained by extending the one given for functions in $\mathcal{O}^{1}(\overline{\mathbb{C}} \backslash[0,1])$.

Remark 5.1. One has

$$
\varphi_{1}(z)=-z\left[\varphi\left(\frac{1}{z}-1\right)-\varphi(-1)\right]+\varphi^{\prime}(-1)+\varphi(z-1)
$$

from which it follows that

$$
z \varphi_{1}\left(\frac{1}{z}\right)=-[\varphi(z-1)-\varphi(-1)]+z \varphi^{\prime}(-1)+z \varphi\left(\frac{1}{z}-1\right)
$$

and

$$
\varphi_{1}(z)+z \varphi_{1}\left(\frac{1}{z}\right)=(1+z)\left(\varphi(-1)+\varphi^{\prime}(-1)\right) .
$$




\subsection{The dilogarithm.}

5.1.1. Let us define

$$
\varphi_{0}(z)=-\frac{1}{\pi} \mathrm{Li}_{2}\left(\frac{1}{z}\right),
$$

where the dilogarithm is taken with its principal branch in $\mathbb{C} \backslash[1,+\infty]$ (see Appendix 3 for a short summary of the properties of the dilogarithm and [O] and references therein for more details). The function $\varphi_{0}$ belongs to $\mathcal{O}^{1}(\overline{\mathbb{C}} \backslash[0,1])$. It is real on the real axis outside $[0,1]$ and its only singular points are 0 and 1 . It is bounded outside of any neighborhood of 0 and

$$
\Im m \varphi_{0}(x \pm i 0)= \pm \log \frac{1}{x}, 0<x \leq 1,
$$

thus the relation with the real Brjuno function $B$ is clear: $B(x)=\left[(1-T)^{-1} f\right](x)$ with $f(x)=\sum_{n \in \mathbb{Z}} \mathfrak{I} m \varphi_{0}(x+i 0-n)$.

5.1.2. Let us now consider

$$
\varphi_{1}=\left[L\left(\begin{array}{ll}
1 & 1 \\
0 & 1
\end{array}\right)+L\left(\begin{array}{ll}
0 & 1 \\
1 & 1
\end{array}\right)\right] \varphi_{0} \in \mathcal{O}^{1}(\overline{\mathbb{C}} \backslash[1 / 2,2]) .
$$

Since $\varphi_{0}(-1)=\pi / 12$ and $\varphi_{0}^{\prime}(-1)=\frac{1}{\pi} \log 2$ one has

$$
\varphi_{1}(z)=\frac{1}{\pi}\left[z \operatorname{Li}_{2}\left(\frac{z}{1-z}\right)-\operatorname{Li}_{2}\left(\frac{1}{z-1}\right)\right]+\frac{\pi}{12} z+\frac{1}{\pi} \log 2 .
$$

The function $\varphi_{1}$ is real. It admits as unique singularities the points $1 / 2,1$ and 2 and has two cuts along $(1 / 2,1)$ and $(1,2)$. It can be continuously extended to $1 / 2$ and 2 and it is bounded outside any neighborhood of 1 . Moreover

$$
\mathfrak{I} m \varphi_{1}(x \pm i 0)= \begin{cases} \pm x \log \frac{x}{1-x} & \text { if } 1 / 2 \leq x<1 \\ \pm \log \frac{1}{x-1} & \text { if } 1<x \leq 2 .\end{cases}
$$

Note that if $1 / 2 \leq x<1$, then

$$
x \log \frac{x}{1-x}=\log \frac{1}{1-x}+x \log x+(1-x) \log (1-x) .
$$

One also has

$$
\varphi_{1}(z)+z \varphi_{1}\left(\frac{1}{z}\right)=(1+z)\left(\frac{\pi}{12}+\frac{1}{\pi} \log 2\right) .
$$

Lemma 5.2. The function $\varphi_{1}(z)+i \log (1-z)$ is continuous on $\overline{\mathbb{H}^{+}}$and its value at 1 is $\frac{1}{\pi} \log 2+\frac{7 \pi}{12}$.

Proof. Applying (A3.7) to (5.7) twice one gets

$$
\begin{aligned}
\varphi_{1}(z) & =\frac{1}{\pi} \log 2-\frac{\pi}{6}+\frac{\pi}{4} z+\frac{1}{\pi}\left[\operatorname{Li}_{2}(z-1)-z \operatorname{Li}_{2}\left(\frac{1-z}{z}\right)\right] \\
& +\frac{1}{2 \pi}\left[\log ^{2}(1-z)-z \log ^{2} \frac{1-z}{-z}\right] .
\end{aligned}
$$


In this expression the function $\operatorname{Li}_{2}(z-1)-z \operatorname{Li}_{2}\left(\frac{1-z}{z}\right)$ is regular and vanishing at $z=1$. Moreover

$$
\log ^{2}(1-z)-z \log ^{2} \frac{1-z}{-z}=\log ^{2}(1-z)-\log ^{2} \frac{1-z}{-z}+(1-z) \log ^{2} \frac{1-z}{-z},
$$

where $(1-z) \log ^{2} \frac{1-z}{-z}$ vanishes at $z=1$, and

$$
\log ^{2}(1-z)-\log ^{2} \frac{1-z}{-z}=-\log ^{2}(-z)+2 \log (-z) \log (1-z) .
$$

In a neighborhood of 1 in $\overline{\mathbb{H}^{+}}$one has $\log (-z)+i \pi=\mathrm{O}(|z-1|)$, thus

$$
\log ^{2}(1-z)-\log ^{2} \frac{1-z}{-z}=\pi^{2}-2 i \pi \log (1-z)+\mathrm{O}\left(|z-1| \log \frac{1}{|z-1|}\right) .
$$

This lemma leads to the following important

Corollary 5.3. The real part of $\varphi_{1}$ is bounded in $\overline{\mathbb{C}} \backslash[1 / 2,2]$. It has an extension to a continuous function on $\overline{\mathbb{C}} \backslash\{1\}$ and

$$
\lim _{x \rightarrow 1^{ \pm}} \mathfrak{R} e \varphi_{1}(x)=\frac{1}{\pi} \log 2+\frac{\pi}{12} \mp \frac{\pi}{2} .
$$

This corollary is the motivation for using $\varphi_{1}$ instead of $\varphi_{0}$ as the starting point of the construction of the complex Brjuno function. Equation (5.2) shows that this leads to the same result.

5.2. A natural compactification of $\mathbb{H}^{+}$. By Lemma 5.2 above, $\mathfrak{R} e \varphi_{1}$ extends continuously to $\overline{\mathbb{H}^{+}} \backslash\{1\}$ with limits at 1 along rays. This means that $\mathfrak{R} e \varphi_{1}$ extends continuously to the compactification of $\mathbb{H}^{+}$obtained from $\overline{\mathbb{H}^{+}}$by blowing out 1 into a semicircle (corresponding to all rays in $\mathbb{H}^{+}$which end in 1). If we want to obtain a similar result for the complex Brjuno function $\sum_{Z \mathcal{M}} \varphi_{0}$ we have to do the same thing at every point of $\overline{\mathbb{Q}}$.

5.2.1. We will consider

$$
\hat{\mathbb{H}}^{+}=\mathbb{H}^{+} \sqcup(\mathbb{R} \backslash \mathbb{Q}) \sqcup\left(\overline{\mathbb{Q}} \times\left[-\frac{\pi}{2},+\frac{\pi}{2}\right]\right)
$$

(where $\overline{\mathbb{Q}}=\mathbb{Q} \cup\{\infty\}$ ) equipped with the topology defined by the following fundamental system of neighborhoods at any point $z \in \hat{\mathbb{H}}^{+}$:

a) if $z_{0} \in \mathbb{H}^{+}$, a fundamental system of neighborhoods is given by $\left\{\left|z-z_{0}\right|<\varepsilon\right\}$, $0<\varepsilon<\Im m z_{0}$

b) if $\alpha_{0} \in \mathbb{R} \backslash \mathbb{Q}$, a fundamental system of neighborhoods is given by the sets $(\varepsilon>0)$

$$
\begin{aligned}
V_{\varepsilon}\left(\alpha_{0}\right) & =\left\{z \in \mathbb{H}^{+},\left|z-\alpha_{0}\right|<\varepsilon\right\} \\
& \cup\left\{\alpha \in \mathbb{R} \backslash \mathbb{Q},\left|\alpha-\alpha_{0}\right|<\varepsilon\right\} \\
& \cup\left\{(\alpha, \theta) \in \mathbb{Q} \times\left[-\frac{\pi}{2},+\frac{\pi}{2}\right],\left|\alpha-\alpha_{0}\right|<\varepsilon\right\} ;
\end{aligned}
$$


c) if $\alpha_{0} \in \mathbb{Q}$, a fundamental system of neighborhoods of $\left(\alpha_{0}, \pi / 2\right)$ (resp. $\left.\left(\alpha_{0},-\pi / 2\right)\right)$ is given by $(0<\varepsilon<\pi)$

$$
\begin{aligned}
V_{\varepsilon}\left(\alpha_{0}, \pi / 2\right) & =\left\{z \in \mathbb{H}^{+},\left|z-\alpha_{0}\right|<\varepsilon, 0<\arg \left(z-\alpha_{0}\right)<\varepsilon\right\} \\
& \cup\left\{\alpha \in \mathbb{R} \backslash \mathbb{Q}, 0<\alpha-\alpha_{0}<\varepsilon\right\} \\
& \cup\left\{(\alpha, \theta) \in \mathbb{Q} \times\left[-\frac{\pi}{2},+\frac{\pi}{2}\right], 0<\alpha-\alpha_{0}<\varepsilon\right\} \\
& \cup\left\{\left(\alpha_{0}, \theta\right), \pi / 2-\varepsilon<\theta \leq \pi / 2\right\}, \\
V_{\varepsilon}\left(\alpha_{0},-\pi / 2\right) & =\left\{z \in \mathbb{H}^{+},\left|z-\alpha_{0}\right|<\varepsilon, \pi>\arg \left(z-\alpha_{0}\right)>\pi-\varepsilon\right\} \\
& \cup\left\{\alpha \in \mathbb{R} \backslash \mathbb{Q}, 0<\alpha_{0}-\alpha<\varepsilon\right\} \\
& \cup\left\{(\alpha, \theta) \in \mathbb{Q} \times\left[-\frac{\pi}{2},+\frac{\pi}{2}\right], 0<\alpha_{0}-\alpha<\varepsilon\right\} \\
& \cup\left\{\left(\alpha_{0}, \theta\right),-\pi / 2 \leq \theta<-\pi / 2+\varepsilon\right\} ;
\end{aligned}
$$

d) if $\alpha_{0} \in \mathbb{Q},-\pi / 2<\theta_{0}<\pi / 2$, a fundamental system of neighborhoods of $\left(\alpha_{0}, \theta_{0}\right)$ is given by $\left(0<\varepsilon<\pi / 2-\left|\theta_{0}\right|\right)$

$$
\begin{aligned}
V_{\varepsilon}\left(\alpha_{0}, \theta_{0}\right) & =\left\{z \in \mathbb{H}^{+},\left|z-\alpha_{0}\right|<\varepsilon, \theta_{0}-\varepsilon<\pi / 2-\arg \left(z-\alpha_{0}\right)<\theta_{0}+\varepsilon\right\} \\
& \cup\left\{\left(\alpha_{0}, \theta\right) \in \mathbb{Q} \times\left[-\frac{\pi}{2},+\frac{\pi}{2}\right], \theta_{0}-\varepsilon<\theta<\theta_{0}+\varepsilon\right\} ;
\end{aligned}
$$

e) a fundamental system of neighborhoods of $(\infty, \pi / 2)(\operatorname{resp} .(\infty,-\pi / 2))$ is given by $(0<\varepsilon<\pi)$

$$
\begin{aligned}
V_{\varepsilon}(\infty, \pi / 2) & =\left\{z \in \mathbb{H}^{+},|z|>\varepsilon^{-1}, \pi>\arg z>\pi-\varepsilon\right\} \\
& \cup\left\{\alpha \in \mathbb{R} \backslash \mathbb{Q}, \alpha<-\varepsilon^{-1}\right\} \\
& \cup\left\{(\alpha, \theta) \in \mathbb{Q} \times\left[-\frac{\pi}{2},+\frac{\pi}{2}\right], \alpha<-\varepsilon^{-1}\right\} \\
& \cup\{(\infty, \theta), \pi / 2 \geq \theta>\pi / 2-\varepsilon\}, \\
V_{\varepsilon}(\infty,-\pi / 2) & =\left\{z \in \mathbb{H}^{+},|z|>\varepsilon^{-1}, 0<\arg z<\varepsilon\right\} \\
\cup & \left\{\alpha \in \mathbb{R} \backslash \mathbb{Q}, \alpha>\varepsilon^{-1}\right\} \\
& \cup\left\{(\alpha, \theta) \in \mathbb{Q} \times\left[-\frac{\pi}{2},+\frac{\pi}{2}\right], \alpha>\varepsilon^{-1}\right\} \\
& \cup\{(\infty, \theta),-\pi / 2 \leq \theta<-\pi / 2+\varepsilon\} ;
\end{aligned}
$$

f) if $-\pi / 2<\theta_{0}<\pi / 2$, a fundamental system of neighborhoods of $\left(\infty, \theta_{0}\right)$ is given by $\left(0<\varepsilon<\pi / 2-\left|\theta_{0}\right|\right)$

$$
\begin{aligned}
V_{\varepsilon}\left(\infty, \theta_{0}\right) & =\left\{z \in \mathbb{H}^{+},|z|>\varepsilon^{-1}, \theta_{0}-\varepsilon<\arg z-\pi / 2<\theta_{0}+\varepsilon\right\} \\
& \cup\left\{(\infty, \theta),\left|\theta-\theta_{0}\right|<\varepsilon\right\} .
\end{aligned}
$$

5.2.2. One can check that the axioms for a system of neighborhoods are verified. The following is the only non-trivial property: if $\zeta \in \hat{\mathbb{H}}^{+}$and $V$ is a neighborhood of $\zeta$, then there exists a neighborhood $W$ of $\zeta$ such that $V$ is a neighborhood of each point of $W$. This must be checked directly for each of the above listed cases.

5.2.3. It is clear that the topology of $\hat{\mathbb{H}}^{+}$induces on $\mathbb{H}^{+}$the usual topology and that $\mathbb{H}^{+}$is an open dense subset of $\hat{\mathbb{H}}^{+}$. 
5.2.4. The space $\hat{\mathbb{H}}^{+}$is compact and Hausdorff. More precisely there exists a homeomorphism of $\overline{\mathbb{D}}$ onto $\hat{\mathbb{H}}^{+}$whose restriction to $\mathbb{D}$ is a homeomorphism onto $\mathbb{H}^{+}$. One can therefore give to $\hat{\mathbb{H}}^{+}$the structure of a topological manifold with boundary; the boundary is $\partial \hat{\mathbb{H}}^{+}=\hat{\mathbb{H}}^{+} \backslash \mathbb{H}^{+}$and is homeomorphic to $\mathbb{S}^{1}$.

5.2.5. The action of $\operatorname{PSL}(2, \mathbb{Z})$ on $\mathbb{H}^{+}$by homographies has a continuous extension to an action on $\hat{\mathbb{H}}^{+}$: just define

$$
\begin{aligned}
\left(\begin{array}{ll}
a & b \\
c & d
\end{array}\right) \cdot \alpha & =\frac{a \alpha+b}{c \alpha+d}, \forall \alpha \in \mathbb{R} \backslash \mathbb{Q} \\
\left(\begin{array}{ll}
a & b \\
c & d
\end{array}\right) \cdot(\alpha, \theta) & =\left(\frac{a \alpha+b}{c \alpha+d}, \theta\right), \forall \alpha \in \overline{\mathbb{Q}}, \forall \theta \in\left[-\frac{\pi}{2},+\frac{\pi}{2}\right] .
\end{aligned}
$$

Let $I$ denote a compact non-trivial interval in $\mathbb{R}$ and $g=\left(\begin{array}{ll}a & b \\ c & d\end{array}\right) \in \operatorname{PGL}(2, \mathbb{Z})$.

Lemma 5.4. Assume that $-d / c \notin I$ (so that $g \cdot I=I^{\prime}$ is also a compact interval of $\mathbb{R})$. If $\varphi \in \mathcal{O}^{1}(\overline{\mathbb{C}} \backslash I)$ has the following properties:

(i) $\varphi$ is real,

(ii) the harmonic function $\mathfrak{R} e \varphi$ on $\mathbb{H}^{+}$has a continuous extension to $\hat{\mathbb{H}}^{+}$, then the function $L_{g} \varphi \in \mathcal{O}^{1}\left(\overline{\mathbb{C}} \backslash I^{\prime}\right)$ also has these two properties.

Proof. It is enough to distinguish three cases:

(a) If $\alpha$ is real and irrational one has

$$
\mathfrak{R} e L_{g} \varphi(\alpha)=(a-c \alpha)\left[\mathfrak{R} e \varphi\left(\frac{d \alpha-b}{a-c \alpha}\right)-\varphi\left(-\frac{d}{c}\right)\right]-\varepsilon_{g} c^{-1} \varphi^{\prime}\left(-\frac{d}{c}\right) .
$$

Note that, from the assumption $-d / c \notin I$, it follows that $\varphi(-d / c)$ and $\varphi^{\prime}(-d / c)$ are both real.

(b) If $\alpha=\infty$ and $\theta \in\left[-\frac{\pi}{2},+\frac{\pi}{2}\right]$ is arbitrary, then $\mathfrak{R} e L_{g} \varphi(\infty, \theta)=0$.

(c) Finally, if $\alpha$ is a rational number and $\theta \in\left[-\frac{\pi}{2},+\frac{\pi}{2}\right]$ is arbitrary, one has

$$
\mathfrak{R} e L_{g} \varphi(\alpha, \theta)=(a-c \alpha)\left[\mathfrak{R} e \varphi\left(\frac{d \alpha-b}{a-c \alpha}, \varepsilon_{g} \theta\right)-\varphi\left(-\frac{d}{c}\right)\right]-\varepsilon_{g} c^{-1} \varphi^{\prime}\left(-\frac{d}{c}\right) .
$$

5.2.6. If $I$ is a non-trivial compact interval of $\mathbb{R}$, we denote by $\hat{\mathcal{C}}_{\mathbb{R}}(\overline{\mathbb{C}} \backslash I)$ the space of holomorphic functions $\varphi \in \mathcal{O}^{1}(\overline{\mathbb{C}} \backslash I)$ which are real and whose real part on $\mathbb{H}^{+}$ extends to a continuous function on $\hat{\mathbb{H}}^{+}$. This is a Banach space with the norm

$$
\begin{aligned}
\|\varphi\|_{\hat{\mathcal{C}}} & :=\sup \left\{|\Re e \varphi(z)|, \quad z \in \mathbb{H}^{+}\right\} \\
& =\sup \left\{|\Re e \varphi(z)|, z \in \hat{\mathbb{H}}^{+}\right\} \\
& =\sup \left\{|\Re e \varphi(z)|, \quad z \in \partial \hat{\mathbb{H}}^{+}\right\}
\end{aligned}
$$

(the equality of all these norms is a trivial consequence of the maximum principle). $\hat{\mathcal{C}}_{\mathbb{R}}(\overline{\mathbb{C}} \backslash I)$ is a real closed vector subspace of the Banach space $E(I)$ of holomorphic functions in $\mathcal{O}^{1}(\overline{\mathbb{C}} \backslash I)$ with bounded real part with respect to the norm

$$
\|\varphi\|_{E(I)}=\sup _{z \in \overline{\mathbb{C}} \backslash I}|\mathfrak{R} e \varphi(z)|
$$

(generalizing the definition used in Section 4.4 for the particular case $I=[0,1]$ ). 
If $g=\left(\begin{array}{ll}a & b \\ c & d\end{array}\right) \in \operatorname{PGL}(2, \mathbb{Z})$ verifies $-d / c \notin I$, then, by Lemma $5.4, L_{g}$ defines an operator of $\hat{\mathcal{C}}_{\mathbb{R}}(\overline{\mathbb{C}} \backslash I)$ into $\hat{\mathcal{C}}_{\mathbb{R}}(\overline{\mathbb{C}} \backslash g \cdot I)$. From its definition it is easy to see that this operator is bounded.

Let us assume now that $I \subset(-1,+\infty), I=\left[\gamma_{0}, \gamma_{1}\right]$. For all $m \geq 1, L_{g(m)}$ defines a bounded operator of $\hat{\mathcal{C}}_{\mathbb{R}}(\overline{\mathbb{C}} \backslash I)$ into $\hat{\mathcal{C}}_{\mathbb{R}}\left(\overline{\mathbb{C}} \backslash\left[1 /\left(m+\gamma_{1}\right), 1 /\left(m+\gamma_{0}\right)\right]\right)$. If $\varphi \in \hat{\mathcal{C}}_{\mathbb{R}}(\overline{\mathbb{C}} \backslash I)$, then

$$
T \varphi=\sum_{m \geq 1} L_{g(m)} \varphi
$$

is holomorphic, real and belongs to $\mathcal{O}^{1}\left(\overline{\mathbb{C}} \backslash\left[0,1 /\left(1+\gamma_{0}\right)\right]\right)$.

Proposition 5.5. The function $T \varphi$ belongs to $\hat{\mathcal{C}}_{\mathbb{R}}\left(\overline{\mathbb{C}} \backslash\left[0,1 /\left(1+\gamma_{0}\right)\right]\right)$ and the operator $T$ from $\hat{\mathcal{C}}_{\mathbb{R}}(\overline{\mathbb{C}} \backslash I)$ into this space is bounded. More precisely, for $k \geq 0$, $2^{k} \leq j<j^{\prime} \leq 2^{k+1}$, one has

$$
\left\|\sum_{j \leq m<j^{\prime}} L_{g(m)} \varphi\right\|_{C} \leq C_{I}(1+k) 2^{-k}\|\varphi\|_{\hat{\mathcal{C}}}
$$

thus the series $\sum \mathfrak{R} e L_{g(m)} \varphi$ is uniformly convergent in $\hat{\mathbb{H}}^{+}$.

Proof. As in the proof of Proposition 4.9, by Poisson's formula one has the estimates

$$
\begin{aligned}
|\varphi(z)| & \leq C_{I}|z|^{-1}\|\varphi\|_{\hat{\mathcal{C}}}, \\
|D \varphi(z)| & \leq C_{I}|z|^{-2}\|\varphi\|_{\hat{\mathcal{C}}}, \\
\left|D^{2} \varphi(z)\right| & \leq C_{I}|z|^{-3}\|\varphi\|_{\hat{\mathcal{C}}},
\end{aligned}
$$

provided that $\operatorname{dist}(z, I) \geq 1$ and $\varphi \in \hat{\mathcal{C}_{\mathbb{R}}}(\overline{\mathbb{C}} \backslash I)$.

Now let $k \geq 0$, and let $j, j^{\prime}$ be such that $2^{k} \leq j<j^{\prime} \leq 2^{k+1}$. Let us also denote $\varphi_{j, j^{\prime}}=\sum_{j \leq m<j^{\prime}} L_{g(m)} \varphi$. Clearly we get $\varphi_{j, j^{\prime}} \in \hat{\mathcal{C}}_{\mathbb{R}}\left(\mathbb{C} \backslash I_{k}\right)$, where $I_{k}=$ $\left[\frac{1}{2^{k+1}-1+\gamma_{1}}, \frac{1}{2^{k}+\gamma_{0}}\right]$. By the maximum principle the supremum of $\left|\mathfrak{R} e \varphi_{j, j^{\prime}}\right|$ on $\hat{\mathbb{H}}^{+}$ is attained at a point of the boundary $\partial \hat{\mathbb{H}}^{+}$of the form $\alpha \in \mathbb{R} \backslash \mathbb{Q}$, or $(\alpha, \vartheta), \alpha \in \mathbb{Q}$, $-\pi / 2 \leq \theta \leq \pi / 2$, such that $\alpha \in I_{k}$. Note that $\sum_{j \leq m<2^{j^{\prime}}}\left|\varphi^{\prime}(-m)\right| \leq C 2^{-k}\|\varphi\|_{\hat{\mathcal{C}}}$ and $\sum_{j \leq m<2^{j^{\prime}}}|\varphi(-m)| \leq C\|\varphi\|_{\hat{\mathcal{C}}}$. If $\alpha$ is irrational and contained in $I_{k}$ one has

$$
\sum_{j \leq m<2^{j^{\prime}}}\left|\mathfrak{R} e \varphi\left(\frac{1}{\alpha}-m\right)\right| \leq C(1+k)\|\varphi\|_{\hat{\mathcal{C}}}
$$

and the same estimate holds if $\alpha \in \mathbb{Q} \cap I_{k}$ for all $\theta \in[-\pi / 2, \pi / 2]$. Since $|\alpha|<$ $C 2^{-k}$ for $\alpha \in I_{k}$ one obtains the desired inequality which also implies all the other properties.

Proposition 4.11 leads to the following

Corollary 5.6. The spectral radius of $T$ on $\hat{\mathcal{C}}_{\mathbb{R}}(\overline{\mathbb{C}} \backslash[0,1])$ is less than or equal to $\frac{\sqrt{5}-1}{2} \cdot \sum_{\mathcal{M}}$ defines a bounded operator on this space.

Proof. $\hat{\mathcal{C}}_{\mathbb{R}}(\overline{\mathbb{C}} \backslash[0,1])$ is a closed subspace of $E([0,1])$. 
5.2.7. One constructs a compactification $\widehat{\mathbb{H}^{+} / \mathbb{Z}}$ of $\mathbb{H}^{+} / \mathbb{Z}$ adding the point $i \infty$ and glueing $(\mathbb{R} \backslash \mathbb{Q}) / \mathbb{Z} \sqcup(\mathbb{Q} / \mathbb{Z} \times[-\pi / 2, \pi / 2])$ in the same way we proceeded for $\hat{\mathbb{H}}^{+}$. One obtains a topological manifold with boundary homeomorphic to $\overline{\mathbb{D}}$. The restriction of this homeomorphism to $\mathbb{H}^{+} / \mathbb{Z}$ is onto $\mathbb{D}^{*}$. The boundary $\partial \widehat{\mathbb{H}^{+} / \mathbb{Z}}=\widehat{\mathbb{H}}{ }^{+} / \mathbb{Z} \backslash$ $\left(\mathbb{H}^{+} / \mathbb{Z} \cup\{i \infty\}\right)$ is once again homeomorphic to $\mathbb{S}^{1}$.

5.2.8. If $I$ is a compact non-trivial interval of $\mathbb{R}$ and $\varphi \in \mathcal{O}^{1}(\overline{\mathbb{C}} \backslash I)$, we defined in (2.5) the 1-periodic holomorphic function $\sum_{Z} \varphi$ on $\mathbb{H}^{+}$which extends continuously to $i \infty$.

Proposition 5.7. Assume that $\varphi \in \hat{\mathcal{C}}_{\mathbb{R}}(\overline{\mathbb{C}} \backslash I)$. Then $\sum_{Z} \varphi$ has the following properties:

(i) $\mathfrak{R} e\left(\sum_{Z} \varphi\right)$ is bounded on $\mathbb{H}^{+}$and the function which it defines on $\mathbb{H}^{+} / \mathbb{Z}$ has a continuous extension to $\widehat{\mathbb{H}^{+} / \mathbb{Z}}$.

(ii) One has

$$
\sup _{\mathbb{H}^{+}}\left|\mathfrak{R} e\left(\sum_{Z} \varphi\right)\right| \leq C \sup _{\mathbb{H}^{+}}|\mathfrak{R} e \varphi| .
$$

We postpone the proof of this proposition after the statement of the two following consequences:

Corollary 5.8. If $\varphi \in \hat{\mathcal{C}}_{\mathbb{R}}(\overline{\mathbb{C}} \backslash[0,1])$, then $\sum_{Z \mathcal{M}} \varphi$ has a bounded real part which extends continuously to $\widehat{\mathbb{H}^{+} / \mathbb{Z}}$ and verifies

$$
\sup _{\mathbb{H}^{+}}\left|\mathfrak{R} e \sum_{Z \mathcal{M}} \varphi\right| \leq C \sup _{\mathbb{H}^{+}}|\mathfrak{R} e \varphi| .
$$

Corollary 5.9. Let $\varphi_{0}(z)=-\frac{1}{\pi} \operatorname{Li}_{2}\left(\frac{1}{z}\right)$; the complex Brjuno function $\mathcal{B}=\sum_{Z \mathcal{M}} \varphi_{0}$ has a bounded real part which extends continuously to $\widehat{\mathbb{H}^{+} / \mathbb{Z}}$.

Proof of Corollary 5.9. Let $\varphi_{1}$ be defined as in (5.6). Then $\varphi_{1} \in \hat{\mathcal{C}}_{\mathbb{R}}(\overline{\mathbb{C}} \backslash[1 / 2,2])$, $T \varphi_{1} \in \hat{\mathcal{C}}_{\mathbb{R}}(\overline{\mathbb{C}} \backslash[0,2 / 3])$ and $\sum_{\mathcal{M}} T \varphi_{1} \in \hat{\mathcal{C}}_{\mathbb{R}}(\overline{\mathbb{C}} \backslash[0,1])$. Thus $\varphi_{1}+\sum_{\mathcal{M}} T \varphi_{1} \in$ $\hat{\mathcal{C}}_{\mathbb{R}}(\overline{\mathbb{C}} \backslash[0,2])$. Applying (5.2) to $\varphi_{0}$ we get the desired result.

Proof of Proposition 5.7. It is not restrictive to assume $I=[0,1]$.

Let us consider the holomorphic function $F_{0}$ associated to the hyperfunction

$$
u(x)=\left\{\begin{array}{cc}
x & \text { if } 0 \leq x \leq 1 / 2 \\
1-x & \text { if } 1 / 2 \leq x \leq 1
\end{array}\right.
$$

One has

$$
F_{0}(z)=\frac{1}{\pi}\left[z \log \frac{z-1 / 2}{z}+(1-z) \log \frac{z-1}{z-1 / 2}\right]
$$

with the principal branch of the logarithm: if $z \notin[0,1]$, then $(z-1 / 2) / z$ and $(z-1) /(z-1 / 2)$ do not belong to $[-\infty, 0]$. 
One can easily check that $\left.F_{0}\right|_{\mathbb{H}^{ \pm}}$extends continuously to $\overline{\mathbb{H}^{ \pm}}$. At infinity one has (with $\tilde{z}=z-1 / 2$ ):

$$
\begin{aligned}
z \log \frac{z-1 / 2}{z} & =\left(\frac{1}{2}+\tilde{z}\right) \log \frac{\tilde{z}}{\tilde{z}+1 / 2} \\
& =-\frac{1}{2}-\frac{1}{8 \tilde{z}}+\mathrm{O}\left(\tilde{z}^{-2}\right) \\
(1-z) \log \frac{z-1}{z-1 / 2} & =\left(\frac{1}{2}-\tilde{z}\right) \log \frac{\tilde{z}-1 / 2}{\tilde{z}} \\
& =\frac{1}{2}-\frac{1}{8 \tilde{z}}+\mathrm{O}\left(\tilde{z}^{-2}\right),
\end{aligned}
$$

thus

$$
F_{0}(z)=-\frac{1}{4 \pi z}+\mathrm{O}\left(z^{-2}\right)
$$

(this could also be checked directly by observing that $\int_{0}^{1} u(x) d x=1 / 4$ ).

It is easy to verify that:

- the real part of $F_{0}$ is bounded in $\overline{\mathbb{C}} \backslash[0,1]$;

- the real part of $\sum_{Z} F_{0}$ is bounded in $\mathbb{H}$.

Let $\varphi \in \hat{\mathcal{C}}_{\mathbb{R}}(\overline{\mathbb{C}} \backslash I)$; consider the unique decomposition

$$
\varphi=c(\varphi) F_{0}+\varphi^{\sharp},
$$

where $\varphi^{\sharp} \in \mathcal{O}^{2}(\overline{\mathbb{C}} \backslash[0,1]) \cap \hat{\mathcal{C}}_{\mathbb{R}}(\overline{\mathbb{C}} \backslash[0,1]), c(\varphi) \in \mathbb{R}$. One has $\left\|\varphi^{\sharp}\right\|_{\hat{\mathcal{C}}} \leq C\left\|_{\varphi}\right\|_{\hat{\mathcal{C}}}$ and $|c(\varphi)| \leq C\|\varphi\|_{\hat{\mathcal{C}}}$, i.e. this decomposition is continuous.

Thus we are led to consider only the case $\varphi \in \mathcal{O}^{2}(\overline{\mathbb{C}} \backslash[0,1]) \cap \hat{\mathcal{C}}_{\mathbb{R}}(\overline{\mathbb{C}} \backslash[0,1])$. But in this case $\sum_{Z} \varphi$ converges uniformly on all domains $\{|\mathfrak{R} e z| \leq A\}$ and this fact immediately leads to the assertions of the proposition.

5.2.9. Note that the topology induced by $\widehat{\mathbb{H}^{+} / \mathbb{Z}}$ on $\mathbb{H}^{+} / \mathbb{Z} \sqcup(\mathbb{R} \backslash \mathbb{Q}) / \mathbb{Z}$ is the same as the topology induced by $\mathbb{C} / \mathbb{Z}$. Therefore the continuity of $\Re e \sum_{Z \mathcal{M}} \varphi_{0}$ on $\widehat{\mathbb{H}+/ \mathbb{Z}}$ implies that the real part $\mathfrak{R} e \sum_{Z \mathcal{M}} \varphi_{0}$ of the complex Brjuno function is continuous on $\mathbb{H}^{+} / \mathbb{Z} \sqcup(\mathbb{R} \backslash \mathbb{Q}) / \mathbb{Z}$ in the usual sense. The value $\mathfrak{R} e \sum_{Z \mathcal{M}} \varphi_{0}\left(\alpha_{0}, \pi / 2\right)$ (resp. $\left(\alpha_{0},-\pi / 2\right)$ ), with $\alpha_{0} \in \mathbb{Q} / \mathbb{Z}$, is the right (resp. left) limit of $\mathfrak{R} e \sum_{Z \mathcal{M}} \varphi_{0}(\alpha)$, as $\alpha \in(\mathbb{R} \backslash \mathbb{Q}) / \mathbb{Z}$ tends to $\alpha_{0}$

Recalling Lemma 5.2, one has

$$
\mathfrak{R} e \varphi_{1}(1, \pi / 2)-\mathfrak{R} e \varphi_{1}(1,-\pi / 2)=-\pi
$$

and more precisely

$$
\mathfrak{R} e \varphi_{1}(1, \theta)=\mathfrak{R} e \varphi_{1}(1,0)-\theta .
$$

If $\alpha_{0} \in \mathbb{Q}, \alpha_{0} \neq 1$, then

$$
\mathfrak{R} e \varphi_{1}\left(\alpha_{0}, \theta\right)=\mathfrak{R} e \varphi_{1}\left(\alpha_{0}, 0\right)
$$

for all $\theta \in[-\pi / 2, \pi / 2]$. Thus by (5.2) one obtains that for all $p / q \in \mathbb{Q}(p \wedge q=1)$

$$
\mathfrak{R} e \sum_{Z \mathcal{M}} \varphi_{0}(p / q, \theta)=\mathfrak{R} e \sum_{Z \mathcal{M}} \varphi_{0}(p / q, 0)-\theta / q .
$$

Thus the real part $\mathfrak{R} e \sum_{Z \mathcal{M}} \varphi_{0}$ of the complex Brjuno function has at each rational $p / q \in \mathbb{Q} / \mathbb{Z}$ a decreasing jump of $\pi / q$. 


\subsection{Boundary behaviour of the imaginary part of the complex Brjuno function.}

5.3.0. Notation. We simply denote by $\|\varphi\|$ the norm (5.13) in the Banach space $E(I)$.

We set $\varphi_{0}(z)=-\frac{1}{\pi} \operatorname{Li}_{2}\left(\frac{1}{z}\right)$ as in (5.4) and $\varphi_{1}$ as in (5.6), thus by (5.2)

$$
\mathcal{B}=\sum_{Z}\left(\varphi_{1}+\sum_{\mathcal{M}} T \varphi_{1}\right)=\sum_{Z \mathcal{M}} \varphi_{0} .
$$

We have $\varphi_{1} \in E([1 / 2,2]), T \varphi_{1} \in E([0,2 / 3])$ and $T^{k} \varphi_{1} \in E([0,1])$ for all $k \geq 2$.

In this section we want to estimate the imaginary part of the 1 -periodic function $\mathcal{B}$ near the real axis. We have

$$
\{|\Im m z| \leq 1 / 2\}=\bigcup_{n \in \mathbb{Z}}(\Delta+n) .
$$

For $r \geq 1, m_{1}, \ldots, m_{r} \geq 1$, we recall the definition of $D\left(m_{1}, \ldots, m_{r}\right)$ given in Section 4.2 , namely the set of $z_{0} \in D$ such that the continued fraction is

$$
z_{i}^{-1}=z_{i+1}+m_{i+1}, \quad 0 \leq i<r, \quad z_{i} \in D, \quad z_{r} \in \Delta .
$$

We also set

$$
\begin{aligned}
H & =H_{0} \cup H_{0}^{\prime}=\Delta \backslash \operatorname{int} D \\
H\left(m_{1}, \ldots, m_{r}\right) & =D\left(m_{1}, \ldots, m_{r}\right) \backslash \operatorname{int}\left(\bigcup_{m_{r+1} \geq 1} D\left(m_{1}, \ldots, m_{r+1}\right)\right) .
\end{aligned}
$$

Then we have

$$
\{|\Im m z| \leq 1 / 2\}=\bigcup_{n \in \mathbb{Z}} \bigcup_{r \geq 0} \bigcup_{m_{1}, \ldots, m_{r} \geq 1}\left[H\left(m_{1}, \ldots, m_{r}\right)+n\right] \sqcup \mathbb{R} \backslash \mathbb{Q},
$$

where the sets in the righ-hand term have disjoint interiors.

5.3.1. A set $H\left(m_{1}, \ldots, m_{r}\right)+n$ meets $\mathbb{R}$ in a unique point, which belongs to $\mathbb{Q}$. Conversely, any rational belongs to exactly two such sets: if $p / q \in \mathbb{Q}$ has continued fraction $p / q=n+1 / m_{1}+1 / m_{2}+\cdots+1 / m_{r}$ with $m_{r} \geq 2$ when $q>1$ (i.e. $r>0$ ), these two sets are $H+n$ and $H(1)+n-1$, if $p / q=n$ and $r=0, H\left(m_{1}, \ldots, m_{r}\right)+n$ and $H\left(m_{1}, \ldots, m_{r}-1,1\right)+n$ if $r>0$. The union of these two sets will be denoted by $V(p / q)$; the boundary of $V(p / q) \cap \mathbb{H}^{+}$is formed by parts of the three horocycles, attached to $p / q, p^{\prime} / q^{\prime}$ and $p^{\prime \prime} / q^{\prime \prime}$ (where $\left[p^{\prime} / q^{\prime}, p^{\prime \prime} / q^{\prime \prime}\right]$ is the Farey interval with "center" $p / q$; when $p / q=n \in \mathbb{Z}$ we have $\left.p^{\prime} / q^{\prime}=n-1, p^{\prime \prime} / q^{\prime \prime}=\infty\right)$, which are deduced from $\mathfrak{I} m z=1 / 2$ by the action of SL $(2, \mathbb{Z})$ (see Figure 4 ).

5.3.2. We plan to compare, when $z \in V(p / q)$, the imaginary part of $\mathcal{B}(z)$ to the truncated real Brjuno function

$$
B_{\text {finite }}(p / q)=\sum_{j=0}^{r-1} \beta_{j-1}(p / q) \log \left[A^{j}(p / q-n)\right]^{-1},
$$



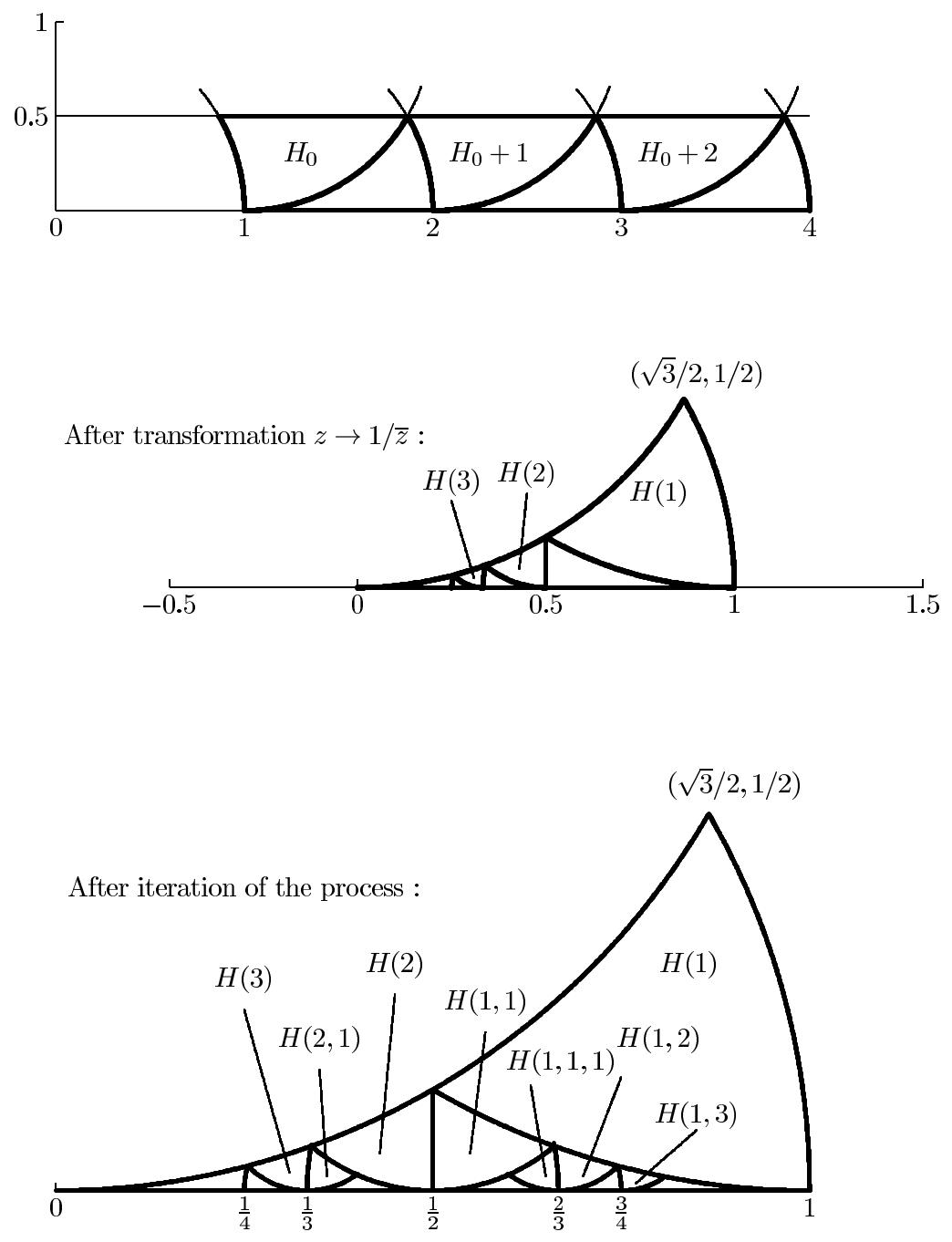

FIGURE 4 . The partition of the strip $0 \leq \Im m z \leq 1 / 2$ by the sets $D\left(m_{1}, \ldots, m_{r}\right)$ and $H\left(m_{1}, \ldots, m_{r}\right)$.

where $p / q$ is as above and $A$ is the Gauss map (A1.1). The point is that we want the dependence on $p / q$ to be explicit in this comparison (i.e. all the constants $c$ are independent of $p / q$ ). This will be achieved by the following

Theorem 5.10. For $k \geq 0, m_{1}, \ldots, m_{k} \geq 1, z_{0} \in H\left(m_{1}, \ldots, m_{k}\right)$ one has

$$
\mathfrak{I} m \mathcal{B}\left(z_{0}\right)=B_{\text {finite }}\left(p_{k} / q_{k}\right)+\left(p_{k-1}-q_{k-1} \mathfrak{R} e z_{0}\right) \mathfrak{I} m \varphi_{1}\left(z_{k}+1\right)+\hat{r}\left(z_{0}\right)
$$

with

$$
\left|\hat{r}\left(z_{0}\right)\right| \leq c q_{k}^{-1}\left|z_{k}\right| \log \left(1+\left|z_{k}\right|^{-1}\right)
$$

for some positive constant $c$ independent of $k, m_{1}, \ldots, m_{k}$. 
The strategy of the proof of the theorem is to start from the formula

$$
\mathfrak{I} m B(z)=\sum_{n \in \mathbb{Z}} \sum_{k \geq 0} \mathfrak{I} m T^{K} \varphi_{1}(z+n),
$$

and to consider each term separately, putting most of them in the remainder part, and replacing the others by simpler expressions. What makes the proof a little lengthy is that while $T^{k} \varphi_{1} \in E([0,1])$ for $k>1$, we have $\varphi_{1} \in E([1 / 2,2])$ and $T \varphi_{1} \in E([0,2 / 3])$, which leads to the need to distinguish several cases (Sections 5.3.3 through 5.3.7) before giving its complete proof (Section 5.3.9).

5.3.3. Recall that $T^{k} \varphi_{1}$ is real and $T^{k} \varphi_{1}(\bar{z})=\overline{T^{k} \varphi_{1}(z)}$, hence $\Im m T^{k} \varphi_{1}(z)$ vanishes on $\mathbb{R}$ outside of

$$
\left\{\begin{array}{cc}
{[1 / 2,2]} & \text { for } k=0 \\
{[0,2 / 3]} & \text { for } k=1 \\
{[0,1]} & \text { for } k>1
\end{array}\right.
$$

As we have, on the other hand,

$$
\left|\varphi^{\prime}(z)\right| \leq C_{K}|z|^{-2}\|\varphi\|
$$

for all $\varphi \in E(I), K \subset \overline{\mathbb{C}} \backslash I$ compact, $z \in K$, we obtain for $z \in \Delta$

$$
\begin{aligned}
& \left|\mathfrak{I} m \varphi_{1}(z+n)\right| \leq c n^{-2}|\mathfrak{I} m z|\left\|\varphi_{1}\right\|, \text { if } n \neq 0,1,2, n \in \mathbb{Z}, \\
& \left|\Im m T \varphi_{1}(z+n)\right| \leq c n^{-2}|\Im m z|\left\|T \varphi_{1}\right\|, \quad \text { if } n \neq-1,0, n \in \mathbb{Z}, \\
& \left|\Im m T^{k} \varphi_{1}(z+n)\right| \leq c n^{-2}|\Im m z||| T^{k} \varphi_{1} \|, \text { if } k>1, n \neq-1,0,1, n \in \mathbb{Z} \text {. }
\end{aligned}
$$

Since, by Proposition 4.11, we have $\left\|T^{k} \varphi_{1}\right\| \leq C k G^{-k}\left\|\varphi_{1}\right\|$, with $G=(\sqrt{5}+1) / 2$, we obtain

Lemma 5.11. For $z \in \Delta \backslash[0,1]$, if we write

$$
\begin{aligned}
& \mathfrak{I} m \mathcal{B}(z)=\mathfrak{I} m \varphi_{1}(z)+\mathfrak{I} m \varphi_{1}(z+1)+\mathfrak{I} m \varphi_{1}(z+2) \\
& \quad+\mathfrak{I} m T \varphi_{1}(z-1)+\mathfrak{I} m T \varphi_{1}(z) \\
& +\sum_{k>1}\left[\mathfrak{I} m T^{k} \varphi_{1}(z-1)+\mathfrak{I} m T^{k} \varphi_{1}(z)+\mathfrak{I} m T^{k} \varphi_{1}(z+1)\right]+r_{0}(z),
\end{aligned}
$$

then we have

$$
\left|r_{0}(z)\right| \leq C|\mathfrak{I} m z|\left\|\varphi_{1}\right\|
$$

5.3.4. One has

$$
\left|\varphi_{1}^{\prime}(z)\right| \leq C \log \left(1+|z-1 / 2|^{-1}\right)
$$

in the neighborhood of $1 / 2$ and

$$
\left|\varphi_{1}^{\prime}(z)\right| \leq C \log \left(1+|z-2|^{-1}\right)
$$

in the neighborhood of 2 . Therefore, for $z \in \Delta \backslash\{0\}$ we have

$$
\left|\mathfrak{I} m \varphi_{1}(z+2)\right| \leq C|\mathfrak{I} m z| \log \left(1+|z|^{-1}\right),
$$

and, for $z \in \Delta \backslash D(1)$

$$
\left|\mathfrak{I} m \varphi_{1}(z)\right| \leq C|\mathfrak{I} m z| \log \left(1+|z-1 / 2|^{-1}\right) .
$$


Next we have

Lemma 5.12. For $k \geq 1$ and $z \in \Delta \backslash\{1\}$ we have

$$
\left|\Im m T^{k} \varphi_{1}(z-1)\right| \leq C|\Im m z| \log \left(1+|z-1|^{-1}\right)\left\|T^{k-1} \varphi_{1}\right\| .
$$

Proof. We have

$$
\left|\mathfrak{I} m T^{k} \varphi_{1}(z)\right|=\left|\mathfrak{I} m\left[z \sum_{m \geq 1}\left(T^{k-1} \varphi_{1}\left(\frac{1}{z}-m\right)-T^{k-1} \varphi_{1}(-m)\right)\right]\right| .
$$

We distinguish two cases:

(a) If $\left|\Im m z^{-1}\right|=|z|^{-2}|\Im m z| \geq 1$, we choose $m_{0} \geq 1$ such that

$$
\left|\frac{1}{z}+m_{0}\right| \leq C|z|^{-2}|\Im m z| \text { and } C^{-1}|z|^{-1} \leq m_{0} \leq C|z|^{-1} \text {. }
$$

One then has

$$
\left|T^{k-1} \varphi_{1}\left(\frac{1}{z}-m\right)-T^{k-1} \varphi_{1}\left(-m-m_{0}\right)\right| \leq C \frac{|\Im m z|\left\|T^{k-1} \varphi_{1}\right\|}{|z|^{2}\left(m+m_{0}\right)^{2}}
$$

from which it follows that

$$
\left|\left[z \sum_{m \geq 1}\left(T^{k-1} \varphi_{1}\left(\frac{1}{z}-m\right)-T^{k-1} \varphi_{1}\left(-m-m_{0}\right)\right)\right] \leq C\right| \mathfrak{I} m z \mid\left\|T^{k-1} \varphi_{1}\right\| .
$$

On the other hand

$$
\left|\mathfrak{I} m z \sum_{1}^{m_{0}} T^{k-1} \varphi_{1}(-m)\right| \leq C|\mathfrak{I} m z| \log \left(1+|z|^{-1}\right)\left\|T^{k-1} \varphi_{1}\right\|,
$$

from which the lemma follows in this case.

(b) If $\left|\Im m z^{-1}\right| \leq 1$ we choose $m_{0} \geq 1$ such that $-m_{0} \leq \Re e 1 / z \leq-m_{0}+1$, thus

$$
\begin{gathered}
\left|\mathfrak{I} m T^{k-1} \varphi_{1}\left(\frac{1}{z}-m\right)\right| \leq C\left|\mathfrak{I} m z\left\|\left.z\right|^{-2}\left(m+m_{0}\right)^{-2}\right\| T^{k-1} \varphi_{1} \|,\right. \\
\left|\mathfrak{R} e\left[T^{k-1} \varphi_{1}\left(\frac{1}{z}-m\right)-T^{k-1} \varphi_{1}\left(-m-m_{0}\right)\right]\right| \leq C\left(m+m_{0}\right)^{-2}\left\|T^{k-1} \varphi_{1}\right\| .
\end{gathered}
$$

We thus obtain

$$
\begin{array}{r}
|\mathfrak{R} e z|\left|\mathfrak{I} m \sum_{m \geq 1} T^{k-1} \varphi_{1}\left(\frac{1}{z}-m\right)\right| \leq C|\mathfrak{I} m z|\left\|T^{k-1} \varphi_{1}\right\|, \\
\left|\mathfrak{R e} \sum_{m \geq 1}\left[T^{k-1} \varphi_{1}\left(\frac{1}{z}-m\right)-T^{k-1} \varphi_{1}\left(-m-m_{0}\right)\right]\right| \leq C|z|\left\|T^{k-1} \varphi_{1}\right\|, \\
\left|\sum_{1}^{m_{0}} T^{k-1} \varphi_{1}(-m)\right| \leq C \log \left(1+|z|^{-1}\right)\left\|T^{k-1} \varphi_{1}\right\|,
\end{array}
$$

which give the desired result. 
Lemma 5.13. 1) If $z \in D(1) \backslash D(1,1)$ we have

$$
\left|\mathfrak{I} m T \varphi_{1}(z)\right| \leq C|\mathfrak{I} m z| \log \left(1+|z-2 / 3|^{-1}\right) .
$$

2) For $k>1, z \in \Delta \backslash\{0\}$ we have

$$
\left|\Im m T^{k} \varphi_{1}(z+1)\right| \leq C|\Im m z| \log \left(1+|z|^{-1}\right)\left\|T^{k-2} \varphi_{1}\right\| \text {. }
$$

Proof. In the first case, in the domain considered, we have $1 / z-1 \in \Delta \backslash D(1)$, hence, by (5.27),

$$
\left|\mathfrak{I} m \varphi_{1}\left(\frac{1}{z}-1\right)\right| \leq C|\mathfrak{I} m z| \log \left(1+|z-2 / 3|^{-1}\right) .
$$

On the other hand we have

$$
\left|\mathfrak{R} e\left[\varphi_{1}\left(\frac{1}{z}-1\right)-\varphi_{1}(-1)\right]\right| \leq 2\left\|\varphi_{1}\right\|,
$$

and for $m \geq 2$

$$
\begin{aligned}
&\left|\varphi_{1}\left(\frac{1}{z}-m\right)-\varphi_{1}(-m)\right| \leq C m^{-2}\left\|\varphi_{1}\right\|, \\
&\left|\mathfrak{I} m \varphi_{1}\left(\frac{1}{z}-m\right)\right| \leq C m^{-2}|\Im m z|\left\|\varphi_{1}\right\|,
\end{aligned}
$$

from which the first inequality of the lemma follows.

In the second case we use Lemma 5.12 to get

$$
\left|\Im m T^{k-1} \varphi_{1}\left(\frac{1}{z+1}-1\right)\right| \leq C|\Im m z| \log \left(1+|z|^{-1}\right)\left\|T^{k-2} \varphi_{1}\right\|,
$$

and deduce the second inequality as above.

5.3.5. For $z \in \Delta, k \geq 1$, we set (as in Section 4.2) $\varepsilon_{k}=\varepsilon_{k}(z)=0$ if $z$ belongs to some $D\left(m_{1}, \ldots, m_{k}\right)$ with $m_{k}=1, \varepsilon_{k}=1$ otherwise.

Starting from Lemma 5.11, we use $(5.26)$ to deal with $\mathfrak{I} m \varphi_{1}(z+2)$, (5.27) to deal with $\mathfrak{I} m \varphi_{1}(z)$ when $\varepsilon=1$, Lemma 5.12 to deal with $\mathfrak{I} m T^{k} \varphi_{1}(z-1)$, and Lemma 5.13 to deal with $\mathfrak{I} m T^{k} \varphi_{1}(z+1)$, and also with $\mathfrak{I} m T \varphi_{1}(z)$ when $\varepsilon_{1}=0$, $\varepsilon_{2}=1$. This gives

Lemma 5.14. For $z \in \Delta \backslash[0,1]$ we have

$$
\begin{aligned}
\mathfrak{I} m \mathcal{B}(z) & =\left(1-\varepsilon_{1}\right) \mathfrak{I} m \varphi_{1}(z)+\mathfrak{I} m \varphi_{1}(z+1)+\left[1-\varepsilon_{2}\left(1-\varepsilon_{1}\right)\right] \mathfrak{I} m T \varphi_{1}(z) \\
& +\sum_{k>1} \mathfrak{I} m T^{k} \varphi_{1}(z)+r_{0}(z)+r_{1}(z)
\end{aligned}
$$

and

$$
\left|r_{1}(z)\right| \leq C|\Im m z| \log \left(1+|\Im m z|^{-1}\right)\left\|\varphi_{1}\right\|
$$

We next recall that, by Proposition 4.1, we have, when $z \in H, k \geq 1$

$$
\left|\Im m T^{k} \varphi_{1}(z)\right| \leq C|z| \log \left(1+|z|^{-1}\right) \sup _{D_{\infty}}\left|T^{k-1} \varphi_{1}\right| \text {. }
$$


5.3.6. In the following two steps, we obtain for $\mathfrak{I} m T^{k} \varphi_{1}(z)$ an approximation similar to Proposition 4.4, first for large $k$ (Proposition 5.15), and then for small $k$ (Proposition 5.16). Here we assume that $k \geq 1, m_{1}, \ldots, m_{k} \geq 1$ and $z_{0} \in$ $D\left(m_{1}, \ldots, m_{k}\right)$ and let $l>0$ and $\varphi=T^{l} \varphi_{1}$.

Proposition 5.15. For $k \geq 1, m_{1}, \ldots, m_{k} \geq 1, z_{0} \in D\left(m_{1}, \ldots, m_{k}\right), l>0$ we have

$$
\begin{aligned}
& \left|\mathfrak{I} m T^{k+l} \varphi_{1}\left(z_{0}\right)-\left[\Im m T^{l} \varphi_{1}\left(z_{k}\right)\right]\left(p_{k-1}-q_{k-1} \mathfrak{\Re} e z_{0}\right)\right| \\
& \quad \leq C q_{k}^{-1}\left|\mathfrak{I} m z_{k}\right| \log \left(1+\left|\mathfrak{I} m z_{k}\right|^{-1}\right) \times\left\{\begin{array}{cl}
\left\|T^{l-2} \varphi_{1}\right\| & \text { if } l>1 \\
\left\|\varphi_{1}\right\| & \text { if } l=1 .
\end{array}\right.
\end{aligned}
$$

Proof. As we did in the proof of Proposition 4.4 we write

$$
\begin{aligned}
T^{k} \varphi\left(z_{0}\right) & =\left(p_{k-1}-q_{k-1} z_{0}\right) \varphi\left(z_{k}\right)+\sum_{j=1}^{k}\left(p_{j-1}-q_{j-1} z_{0}\right) T^{k-j} \varphi\left(z_{j}-1\right) \\
& +\sum_{j=1}^{k}\left(p_{j-1}-q_{j-1} z_{0}\right) \varepsilon_{j} T^{k-j} \varphi\left(z_{j}+1\right) \\
& +\sum_{j=1}^{k}\left(p_{j-2}-q_{j-2} z_{0}\right) R^{\left(m_{j}\right)}\left(T^{k-j} \varphi\right)\left(z_{j-1}\right) \\
& =\left(p_{k-1}-q_{k-1} z_{0}\right) \varphi\left(z_{k}\right)+\hat{R}_{1}^{[k]}(\varphi)\left(z_{0}\right)+\hat{R}_{2}^{[k]}(\varphi)\left(z_{0}\right)+\hat{R}_{0}^{[k]}(\varphi)\left(z_{0}\right),
\end{aligned}
$$

where

$$
\begin{aligned}
R^{\left(m_{j}\right)}(\psi)\left(z_{j-1}\right) & =\sum_{m \geq 1} \psi^{\prime}(-m)+z_{j-1} \sum_{m \geq 1,\left|m-m_{j}\right| \leq 1} \psi(-m) \\
& -z_{j-1} \sum_{m \geq 1,\left|m-m_{j}\right|>1}\left[\psi\left(z_{j}+m_{j}-m\right)-\psi(-m)\right] .
\end{aligned}
$$

We will use repeatedly the following inequalities:

$$
\begin{aligned}
\left|\mathfrak{I} m\left(w_{1} w_{2}\right)\right| & \leq\left|w_{1}\right|\left|\mathfrak{I} m w_{2}\right|+\left|w_{2}\right|\left|\mathfrak{I} m w_{1}\right|, \\
\left|p_{j-1}-q_{j-1} z_{0}\right| & \leq c q_{j}^{-1}, \\
\left|\mathfrak{I} m\left(p_{j-1}-q_{j-1} z_{0}\right)\right| & =q_{j-1}\left|\mathfrak{I} m z_{0}\right| \leq C q_{j-1} q_{k}^{-2}\left|\Im m z_{k}\right| .
\end{aligned}
$$

By Proposition 4.1, as $l>0$, for $1 \leq j \leq k$ we have

$$
\left|T^{k-j} \varphi\left(z_{j}-1\right)\right| \leq C \sup _{D_{\infty}}\left|T^{k-j+l-1} \varphi_{1}\right|
$$

and, by Lemma 5.12,

$$
\left|\mathfrak{I} m T^{k-j} \varphi\left(z_{j}-1\right)\right| \leq C\left|\mathfrak{I} m z_{j}\right| \log \left(1+\left|z_{j}-1\right|^{-1}\right)\left\|T^{k-j+l-1} \varphi_{1}\right\| .
$$

We observe that for $j<k-1$

$$
\log \left(1+\left|z_{j}-1\right|^{-1}\right) \leq C \log \left(1+\left|z_{j+1}\right|^{-1}\right) \leq C \log \left(1+m_{j+2}\right),
$$

and one also has

$$
\begin{aligned}
& \log \left(1+\left|z_{k-1}-1\right|^{-1}\right) \leq C \log \left(1+\left|z_{k}\right|^{-1}\right) \leq C \log \left(1+\left|\Im m z_{k}\right|^{-1}\right), \\
& \log \left(1+\left|z_{k}-1\right|^{-1}\right) \leq \log \left(1+\left|\mathfrak{I} m z_{k}\right|^{-1}\right) \text {. }
\end{aligned}
$$


Thus, from (5.31)-(5.35) we get

$$
\left|\Im m \hat{R}_{1}^{[k]}(\varphi)\left(z_{0}\right)\right| \leq C q_{k}^{-1}\left|\Im m z_{k}\right| \log \left(1+\left|\Im m z_{k}\right|^{-1}\right)\left\|T^{l-1} \varphi_{1}\right\| .
$$

Similarly, by Lemma 5.13, we have, for $j<k, l \geq 1$ or $j \leq k, l>1$

$$
\left|\mathfrak{I} m T^{k-j} \varphi\left(z_{j}+1\right)\right| \leq C\left|\mathfrak{I} m z_{j}\right| \log \left(1+\left|z_{j}\right|^{-1}\right)\left\|T^{k-j+l-2} \varphi_{1}\right\|,
$$

and, by Proposition 4.2,

$$
\left|T^{k-j} \varphi\left(z_{j}+1\right)\right| \leq C \sup _{D_{\infty}}\left|T^{k-j+l-2} \varphi_{1}\right|
$$

On the other hand, when $j=k, l=1$ (i.e. $T^{k-j} \varphi=T \varphi_{1}$ ) one has

$$
\begin{aligned}
\left|\mathfrak{I} m T \varphi_{1}\left(z_{k}+1\right)\right| & \leq C\left|\mathfrak{I} m z_{k}\right|\left\|T \varphi_{1}\right\|, \\
\left|T \varphi_{1}\left(z_{k}+1\right)\right| & \leq C\left\|T \varphi_{1}\right\|
\end{aligned}
$$

(note that as $T \varphi_{1} \in E([0,2 / 3]), z_{k}+1$ is well separated from the boundary). This gives

$$
\left|\Im m \hat{R}_{2}^{[k]}(\varphi)\left(z_{0}\right)\right| \leq C q_{k}^{-1}\left|\Im m z_{k}\right| \log \left(1+\left|z_{k}\right|^{-1}\right) \times\left\{\begin{array}{cc}
\left\|T^{l-2} \varphi_{1}\right\| & (l>1) \\
\left\|\varphi_{1}\right\| & (l=1)
\end{array}\right.
$$

Finally one has

$$
\begin{aligned}
\left|R^{\left(m_{j}\right)}(\psi)\left(z_{j-1}\right)\right| & \leq C\|\psi\| \\
\left|\mathfrak{I} m R^{\left(m_{j}\right)}(\psi)\left(z_{j-1}\right)\right| & \leq C\|\psi\|\left[\left|\mathfrak{I} m z_{j-1}\right| \log \left(1+m_{j}\right)+\mathfrak{R} e z_{j-1}\left|\mathfrak{I} m z_{j}\right|\right] \\
& \leq c m_{j}^{-1}\left|\mathfrak{I} m z_{j}\right|\|\psi\|
\end{aligned}
$$

giving

$$
\left|\Im m R_{0}^{[k]}(\varphi)\left(z_{0}\right)\right| \leq c q_{k}^{-1}\left|\Im m z_{k}\right|\left\|T^{l} \varphi_{1}\right\| .
$$

To conclude our proof we only need to observe that

$$
\left|\mathfrak{I} m\left(p_{k-1}-q_{k-1} z_{0}\right) \mathfrak{R} e \varphi\left(z_{k}\right)\right| \leq C q_{k}^{-1}\left|\Im m z_{k}\right| \mid T^{l} \varphi_{1} \|,
$$

to get the desired result.

5.3.7. For $k \geq 1, m_{1}, \ldots, m_{k} \geq 1, z_{0} \in D\left(m_{1}, \ldots, m_{k}\right)$ we will now consider $\Im m T^{k} \varphi_{1}\left(z_{0}\right)$ (i.e. the case $l=0$ left out from Proposition 5.15):

Proposition 5.16. For $k \geq 1, m_{1}, \ldots, m_{k} \geq 1, z_{0} \in D\left(m_{1}, \ldots, m_{k}\right)$ we have

$$
\begin{aligned}
& \left|\mathfrak{I} m T^{k} \varphi_{1}\left(z_{0}\right)-\left(p_{k-1}-q_{k-1} \mathfrak{R} e z_{0}\right)\left[\left(1-\varepsilon_{k+1}\right) \mathfrak{I} m \varphi_{1}\left(z_{k}\right)+\varepsilon_{k} \mathfrak{I} m \varphi_{1}\left(z_{k}+1\right)\right]\right| \\
& \leq C q_{k}^{-1}\left|\mathfrak{I} m z_{k}\right| \log \left(1+\left|\mathfrak{I} m z_{k}\right|^{-1}\right)\left\|\varphi_{1}\right\| .
\end{aligned}
$$

Proof. We now write (with $\varepsilon_{k}^{\prime}=0$ if $m_{k} \leq 2, \varepsilon_{k}^{\prime}=1$ otherwise)

$$
\begin{aligned}
T^{k} \varphi_{1}\left(z_{0}\right) & =\left(p_{k-1}-q_{k-1} z_{0}\right)\left(\varphi_{1}\left(z_{k}\right)+\varepsilon_{k} \varphi_{1}\left(z_{k}+1\right)+\varepsilon_{k}^{\prime} \varphi_{1}\left(z_{k}+2\right)\right) \\
& +\sum_{j=1}^{k-1}\left(p_{j-1}-q_{j-1} z_{0}\right) T^{k-j} \varphi_{1}\left(z_{j}-1\right) \\
& +\sum_{j=1}^{k-1}\left(p_{j-1}-q_{j-1} z_{0}\right) \varepsilon_{j} T^{k-j} \varphi_{1}\left(z_{j}+1\right) \\
& +\sum_{j=1}^{k}\left(p_{j-2}-q_{j-2} z_{0}\right) \tilde{R}^{\left(m_{j}\right)}\left(T^{k-j} \varphi_{1}\right)\left(z_{j-1}\right),
\end{aligned}
$$


where $\tilde{R}^{\left(m_{j}\right)}\left(T^{k-j} \varphi_{1}\right)=R^{\left(m_{j}\right)}\left(T^{k-j} \varphi_{1}\right)$ for $j<k$ but

$$
\begin{aligned}
\tilde{R}^{\left(m_{k}\right)}\left(\varphi_{1}\right)\left(z_{k-1}\right) & =\sum_{m \geq 1} \varphi_{1}^{\prime}(-m)+z_{k-1} \sum_{m \geq 1,\left|m-m_{k}+1\right| \leq 1} \varphi_{1}(-m) \\
& -z_{k-1} \sum_{m \geq 1,\left|m-m_{k}+1\right|>1}\left[\varphi_{1}\left(z_{k}+m_{k}-m\right)-\varphi_{1}(-m)\right] .
\end{aligned}
$$

For the last sum $\tilde{R}_{0}^{[k]}\left(\varphi_{1}\right)$ in $(5.43)$, we have as in (5.39)-(5.41)

$$
\left|\Im m \tilde{R}_{0}^{[k]}\left(\varphi_{1}\right)\left(z_{0}\right)\right| \leq C q_{k}^{-1}\left|\Im m z_{k}\right| \mid \varphi_{1} \| .
$$

The first sum $R_{1}^{[k]}\left(\varphi_{1}\right)$ is dealt with as in (5.31)-(5.35) to get

$$
\left|\Im m R_{1}^{[k]}\left(\varphi_{1}\right)\left(z_{0}\right)\right| \leq C q_{k}^{-1}\left|\Im m z_{k}\right| \log \left(1+\left|\Im m z_{k}\right|^{-1}\right)\left\|\varphi_{1}\right\| .
$$

The middle sum $R_{2}^{[k]}\left(\varphi_{1}\right)$ satisfies the same estimate, proved as in (5.36)-(5.38).

By (5.26) we have

$$
\left|\Im m\left[\left(p_{k-1}-q_{k-1} z_{0}\right) \varphi_{1}\left(z_{k}+2\right)\right]\right| \leq q_{k}^{-1}\left[C\left|\Im m z_{k}\right| \log \left(1+\left|z_{k}\right|^{-1}\right)+1\right]\left\|\varphi_{1}\right\| .
$$

Similarly, if $z_{k} \notin D(1)$ (i.e. if $\varepsilon_{k+1}=1$ ) we have

$$
\left|\Im m\left[\left(p_{k-1}-q_{k-1} z_{0}\right) \varphi_{1}\left(z_{k}\right)\right]\right| \leq C q_{k}^{-1}\left|\Im m z_{k}\right| \log \left(1+\left|z_{k}-1 / 2\right|^{-1}\right)\left\|\varphi_{1}\right\|,
$$
according to (5.27). Finally, for $\varepsilon=0,1$

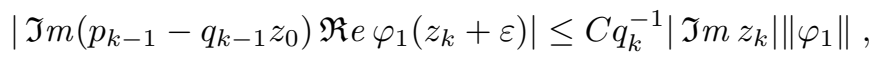

and from (5.43)-(5.48) we get our result.

5.3.8. Starting from Lemma 5.14, we now make use of Propositions 5.15 and 5.16 to obtain a simpler approximation for $\mathfrak{I} m \mathcal{B}(z)$.

Proposition 5.17. For $k \geq 0, m_{1}, \ldots, m_{k} \geq 1, z_{0} \in H\left(m_{1}, \ldots, m_{k}\right)$ we have

$$
\begin{aligned}
\mathfrak{I} m \mathcal{B}\left(z_{0}\right) & =\sum_{l=0}^{k} \Im m \varphi_{1}\left(z_{l}+1\right)\left(p_{l-1}-q_{l-1} \mathfrak{R} e z_{0}\right) \varepsilon_{l} \\
& +\sum_{l=0}^{k-1} \mathfrak{I} m \varphi_{1}\left(z_{l}\right)\left(p_{l-1}-q_{l-1} \mathfrak{R} e z_{0}\right)\left(1-\varepsilon_{l+1}\right)+r\left(z_{0}\right)
\end{aligned}
$$

with $\varepsilon_{0}=1$, and

$$
\left|r\left(z_{0}\right)\right| \leq C q_{k}^{-1}\left|z_{k}\right| \log \left(1+\left|z_{k}\right|^{-1}\right) .
$$

Proof. First, assume $z_{0} \in H$. Then, from (5.28) and Lemma 5.14 we get

$$
\Im m \mathcal{B}(z)=\left(1-\varepsilon_{1}\right) \Im m \varphi_{1}\left(z_{0}\right)+\Im m \varphi_{1}\left(z_{0}+1\right)+r\left(z_{0}\right),
$$

with

$$
\left|r\left(z_{0}\right)\right| \leq C\left|z_{0}\right| \log \left(1+\left|z_{0}\right|^{-1}\right) .
$$

As we also have, for $z_{0} \in H$,

$$
\left|\Im m \varphi_{1}\left(z_{0}\right)\right| \leq C\left|\Im m z_{0}\right|,
$$

we obtain

$$
\left|\Im m \mathcal{I}(z)-\Im m \varphi_{1}\left(z_{0}+1\right)\right| \leq C\left|z_{0}\right| \log \left(1+\left|z_{0}\right|^{-1}\right) .
$$


Next consider $z_{0} \in H\left(m_{1}, \ldots, m_{k}\right)$ with $k \geq 1, m_{1}, \ldots, m_{k} \geq 1$. Observe that

$$
\left[1-\varepsilon_{2}\left(1-\varepsilon_{1}\right)\right]\left(1-\varepsilon_{2}\right)=1-\varepsilon_{2}, \quad\left[1-\varepsilon_{2}\left(1-\varepsilon_{1}\right)\right] \varepsilon_{1}=\varepsilon_{1} .
$$

In the terms which appear in the right-hand term of Lemma 5.14, we use Proposition 5.15 to deal with $\mathfrak{I} m T^{l} \varphi_{1}\left(z_{0}\right), l>k$, and Proposition 5.16 to deal with $\mathfrak{I} m T^{l} \varphi_{1}\left(z_{0}\right), 0<l \leq k$. We have

$$
\begin{aligned}
\mid \sum_{l>k} \Im m T^{l} \varphi_{1}\left(z_{0}\right) & -\left(p_{k-1}-q_{k-1} z_{0}\right) \sum_{l>0} \Im m T^{l} \varphi_{1}\left(z_{k}\right) \mid \\
& \leq C q_{k}^{-1}\left|\Im m z_{k}\right| \log \left(1+\left|\mathfrak{I} m z_{k}\right|^{-1}\right)
\end{aligned}
$$

but using (5.28) (since $z_{k} \in H$ ) we obtain

$$
\left|\sum_{l>k} \mathfrak{I} m T^{l} \varphi_{1}\left(z_{0}\right)\right| \leq C q_{k}^{-1}\left|z_{k}\right| \log \left(1+\left|z_{k}\right|^{-1}\right) .
$$

For $0 \leq l \leq k$, we have in Proposition 5.16 and Lemma 5.14

$$
q_{l}^{-1}\left|\mathfrak{I} m z_{l}\right| \log \left(1+\left|\mathfrak{I} m z_{l}\right|^{-1}\right) \leq C q_{l} q_{k}^{-2}\left|\mathfrak{I} m z_{k}\right| \log \left(1+\left|\mathfrak{I} m z_{l}\right|^{-1}\right)
$$

and

$$
\begin{aligned}
\sum_{0}^{k} q_{l} q_{k}^{-1} \log \left(1+\left|\Im m z_{l}\right|^{-1}\right) & \leq C \sum_{0}^{k} q_{l} q_{k}^{-1} \log \left(1+C q_{l}^{-2} q_{k}^{2}\left|\Im m z_{k}\right|^{-1}\right) \\
& \leq C \log \left(1+\left|\Im m z_{k}\right|^{-1}\right) .
\end{aligned}
$$

5.3.9. We can finally complete the proof of Theorem 5.10.

Let $k \geq 0, m_{1}, \ldots, m_{k} \geq 1$. As we already know, the domain $H\left(m_{1}, \ldots, m_{k}\right)$ meets $\mathbb{R}$ in a unique point which is $p_{k} / q_{k}$. Let us denote $x_{0}=p_{k} / q_{k}$ and consider its continued fraction

$$
x_{i}^{-1}=m_{i+1}+x_{i+1}, \quad 0 \leq i<k, x_{k}=0
$$

(of course $x_{i}$ is the point of intersection of $H\left(m_{i+1}, \ldots, m_{k}\right)$ with $\mathbb{R}$ ).

Let $z_{0} \in H\left(m_{1}, \ldots, m_{k}\right), \mathfrak{I} m z_{0}>0$. We will then have $(-1)^{l} \mathfrak{I} m z_{l}>0$, $0 \leq l \leq k$. On the other hand one has, for $0 \leq l<k$,

$$
\varepsilon_{l} \mathfrak{I} m \varphi_{1}\left(x_{l}+1+(-1)^{l} i 0\right)=(-1)^{l} \varepsilon_{l} \log \frac{1}{x_{l}},
$$

and for $0 \leq l<k-1$

$$
\left(1-\varepsilon_{l+1}\right) \mathfrak{I} m \varphi_{1}\left(x_{l}+(-1)^{l} i 0\right)=\left(1-\varepsilon_{l+1}\right)(-1)^{l} x_{l} \log \frac{1}{x_{l+1}}
$$

since $\frac{x_{l}}{1-x_{l}}=\frac{1}{x_{l+1}}$ when $1-\varepsilon_{l+1} \neq 0$. (5.54) and (5.55) imply that

$$
\begin{aligned}
\sum_{0}^{k-1} & \Im \\
& m \varphi_{1}\left(x_{l}+1+(-1)^{l} i 0\right)\left(p_{l-1}-q_{l-1} x_{0}\right) \varepsilon_{l} \\
& +\sum_{0}^{k-2} \Im m \varphi_{1}\left(x_{l}+(-1)^{l} i 0\right)\left(p_{l-1}-q_{l-1} x_{0}\right)\left(1-\varepsilon_{l+1}\right) \\
& =\sum_{0}^{k-1} \beta_{l-1}\left(x_{0}\right) \log \frac{1}{x_{l}}:=B_{\text {finite }}\left(p_{k} / q_{k}\right) .
\end{aligned}
$$


When we set this expression in Theorem 5.10, and compare the result with the expression for $\mathfrak{I} m B\left(z_{0}\right)$ given by Proposion 5.17 , we see that we have to deal with the following expressions. For $0 \leq l \leq k-1$, and $\varepsilon_{l}=1$ :

$\mathbf{A}_{l}=\mathfrak{I} m \varphi_{1}\left(z_{l}+1\right)\left(p_{l-1}-q_{l-1} \mathfrak{R} e z_{0}\right)-\mathfrak{I} m \varphi_{1}\left(x_{l}+1+(-1)^{l} i 0\right)\left(p_{l-1}-q_{l-1} x_{0}\right)$.

For $0 \leq l \leq k-2$, and $\varepsilon_{l+1}=0$ :

$$
\mathbf{B}_{l}=\mathfrak{I} m \varphi_{1}\left(z_{l}\right)\left(p_{l-1}-q_{l-1} \mathfrak{R} e z_{0}\right)-\mathfrak{I} m \varphi_{1}\left(x_{l}+(-1)^{l} i 0\right)\left(p_{l-1}-q_{l-1} x_{0}\right) .
$$

And when $\varepsilon_{k}=0$ :

$$
\mathbf{C}_{k}=\mathfrak{I} m \varphi_{1}\left(z_{k}+1\right)\left(p_{k-1}-q_{k-1} \mathfrak{R} e z_{0}\right)-\mathfrak{I} m \varphi_{1}\left(z_{k-1}\right)\left(p_{k-2}-q_{k-2} \mathfrak{R} e z_{0}\right) .
$$

First of all one has

$$
\left|\Re e z_{0}-x_{0}\right| \leq C q_{k}^{-2}\left|z_{k}\right|
$$

On the other hand, near 1 one has

$$
\left|\varphi_{1}^{\prime}(z)\right| \leq C|z-1|^{-1}
$$

For $0 \leq l<k$ (resp. $0 \leq l<k-1$ ) the distances of $x_{l}$ and $z_{l}$ from 0 (resp. 1) are comparable. Thus, for $0 \leq l<k$

$$
\left|\Im m \varphi_{1}\left(z_{l}+1\right)-\Im m \varphi_{1}\left(x_{l}+1+(-1)^{l} i 0\right)\right| \leq C x_{l}^{-1}\left|z_{l}-x_{l}\right|,
$$

and for $0 \leq l<k-1$

$$
\left|\Im m \varphi_{1}\left(z_{l}\right)-\Im m \varphi_{1}\left(x_{l}+(-1)^{l} i 0\right)\right| \leq C\left|x_{l}-1\right|^{-1}\left|z_{l}-x_{l}\right| .
$$

We have here $\left|z_{l}-x_{l}\right| \leq C\left|z_{k}\right| q_{l}^{2} q_{k}^{-2}, x_{l}^{-1} \leq C m_{l+1}$ and $\left|x_{l}-1\right|^{-1} \leq c x_{l+1}^{-1} \leq c m_{l+2}$. We thus get, taking (5.54) and (5.55) into account,

$$
\begin{array}{rl}
\left|\mathbf{A}_{l}\right| & \leq C\left(q_{l-1} q_{k}^{-2}\left|z_{k}\right| \log \frac{1}{x_{l}}+x_{l}^{-1}\left|z_{l}-x_{l}\right| q_{l}^{-1}\right) \\
& \leq C\left|z_{k}\right| q_{k}^{-2}\left(q_{l-1} \log m_{l+1}+q_{l} m_{l+1}\right) \\
& \leq C\left|z_{k}\right| q_{k}^{-2} q_{l+1}, \\
\left|\mathbf{B}_{l}\right| \leq C & C\left(q_{l-1} q_{k}^{-2}\left|z_{k}\right| \log \frac{1}{x_{l+1}}+\left|x_{l}-1\right|^{-1}\left|z_{l}-x_{l}\right| q_{l}^{-1}\right) \\
\leq C\left|z_{k}\right| q_{k}^{-2}\left(q_{l-1} \log m_{l+2}+q_{l} m_{l+2}\right) \\
\leq C\left|z_{k}\right| q_{k}^{-2} q_{l+2} .
\end{array}
$$

We thus have

$$
\sum_{l=0}^{k-1}\left|\mathbf{A}_{l}\right|+\sum_{l=0}^{k-2}\left|\mathbf{B}_{l}\right| \leq C\left|z_{k}\right| q_{k}^{-1} .
$$

Finally we note that when $m_{k}=1$, i.e. $\varepsilon_{k}=0$, one has $z_{k-1}^{-1}=1+z_{k}$. Thus

$$
\varphi_{1}\left(z_{k-1}\right)=\left(1+z_{k-1}\right)\left(\frac{\pi}{12}+\frac{1}{\pi} \log 2\right)-z_{k-1} \varphi_{1}\left(1+z_{k}\right)
$$

which gives

$$
\begin{aligned}
\mathbf{C}_{k} & =\Im m \varphi_{1}\left(z_{k}+1\right)\left[\left(p_{k-1}-q_{k-1} \mathfrak{R} e z_{0}\right)+\left(p_{k-2}-q_{k-2} \mathfrak{R} e z_{0}\right) \mathfrak{R} e z_{k-1}\right] \\
& +\left(p_{k-2}-q_{k-2} \mathfrak{R} e z_{0}\right) \Im m z_{k-1}\left(\frac{\pi}{12}+\frac{1}{\pi} \log 2-\mathfrak{R} e \varphi_{1}\left(1+z_{k}\right)\right) .
\end{aligned}
$$


But we have the inequalities

$$
\begin{aligned}
& \left|\frac{\pi}{12}+\frac{1}{\pi} \log 2-\mathfrak{R e} \varphi_{1}\left(1+z_{k}\right)\right| \leq C, \\
& \left|\left(p_{k-2}-q_{k-2} \Re e z_{0}\right) \mathfrak{I} m z_{k-1}\right| \leq C q_{k-1}^{-1}\left|\Im m z_{k-1}\right| \\
& \leq C q_{k}^{-1}\left|\Im m z_{k}\right| \\
& \left|\left(p_{k-1}-q_{k-1} \mathfrak{R} e z_{0}\right)+\left(p_{k-2}-q_{k-2} \mathfrak{R} e z_{0}\right) \mathfrak{R} e z_{k-1}\right| \leq q_{k-2}\left|\mathfrak{I} m z_{0}\right|\left|\Im m z_{k-1}\right| \\
& \leq C q_{k}^{-1}\left|\Im m z_{k}\right| \\
& \left|\Im m \varphi_{1}\left(z_{k}+1\right)\right| \leq C \log \left(1+\left|z_{k}\right|^{-1}\right),
\end{aligned}
$$

hence

$$
\left|\mathbf{C}_{k}\right| \leq C q_{k}^{-1}\left|\mathfrak{I} m z_{k}\right| \log \left(1+\left|z_{k}\right|^{-1}\right),
$$

and the proof of Theorem 5.10 is complete.

Remark 5.18. We recall that the term involved in Theorem 5.10 satisfies

$$
\left(p_{k-1}-q_{k-1} \mathfrak{R} e z_{0}\right) \mathfrak{I} m \varphi_{1}\left(z_{k}+1\right)=\left(q_{k}^{-1} \log \frac{1}{\left|z_{k}\right|}\right)(1+\mathrm{o}(1)),
$$

as $z_{k} \rightarrow 0$.

5.3.10. Here we consider the imaginary part of $\mathcal{B}$ near Brjuno numbers. For $H>0$ let

$$
W_{H}=\left\{w \in \mathbb{H}, \quad \mathfrak{I} m w \geq|\mathfrak{R} e w|^{H}\right\}
$$

and for $0<h<1 / 2$ let

$$
\tilde{W}_{h}=\left\{w \in \mathbb{H}, \quad \Im m w \geq \exp \left[-|\mathfrak{R} e w|^{-h}\right]\right\} .
$$

Then we have

Theorem 5.19. 1) For any Brjuno number $\alpha$ and any $H>0$ we have

$$
\lim _{w \rightarrow 0, w \in W_{H}} \mathfrak{I} m \mathcal{B}(w+\alpha)=B(\alpha) .
$$

2) Let $\alpha$ be an irrational diophantine number and $0<h<1 / 2$ such that

$$
\liminf _{q \rightarrow \infty}\|q \alpha\|_{\mathbb{Z}} q^{1 / h-1}=+\infty
$$

where \|\|$_{\mathbb{Z}}$ denotes the distance from the nearest integer. Then

$$
\lim _{w \rightarrow 0, w \in \tilde{W}_{h}} \mathfrak{I} m \mathcal{B}(w+\alpha)=B(\alpha) .
$$

Proof. We begin by stating a useful lemma (whose proof is an easy adaptation of the arguments of 5.3.9 and is left to the reader).

Lemma 5.20. Let $k \geq 1$ and $m_{1}, \ldots, m_{k} \geq 1$, and let $p_{k} / q_{k}$ be the point of intersection of $H\left(m_{1}, \ldots, m_{k}\right)$ with $\mathbb{R}$. For all $x \in D\left(m_{1}, \ldots, m_{k}\right) \cap \mathbb{R}$ one has

$$
\left|B_{\text {finite }}\left(p_{k} / q_{k}\right)-\sum_{0}^{k-1} \beta_{l-1}(x) \log \frac{1}{x_{l}}\right| \leq C x_{k} q_{k}^{-1},
$$

where $\left(x_{i}\right)_{i \geq 0}$ is the continued fraction of $x$. 
Assume now that $\alpha \in(0,1)$ is irrational with continued fraction

$$
\alpha=1 / m_{1}+1 / m_{2}+\cdots+1 / m_{k}+\cdots .
$$

Let $\left(p_{k} / q_{k}\right)_{k \geq 0}$ denote the sequence of the partial fractions. Let $w$ be a point close to 0 and $z=\alpha+w$. For $|\Im m z| \leq 1 / 2, z$ belongs to a domain $V(p / q)$ (defined in Section 5.3.1) and we distinguish two cases.

(I) Here we assume that $p / q=p_{k} / q_{k}$ is one of the partial fractions of $\alpha$. One then has by Proposition A1.1

$$
\left|\alpha-p_{k} / q_{k}\right| \geq\left(2 q_{k} q_{k+1}\right)^{-1}
$$

(I.1) If $w \in W_{H}$ one gets

$$
\left|z-p_{k} / q_{k}\right| \geq c^{-1}\left(q_{k} q_{k+1}\right)^{-H}
$$

thus

$$
\left|z_{k}\right|^{-1} \leq c q_{k}^{-2}\left(q_{k} q_{k+1}\right)^{H}
$$

and

$$
\frac{1}{q_{k}} \log \left|z_{k}\right|^{-1} \leq q_{k}^{-1}\left[c+H \log q_{k+1}+(H-2) \log q_{k}\right],
$$

which is small when $\alpha$ is a Brjuno number and $k$ is large. Lemma 5.20, Remark 5.18, and Theorem 5.10 lead to the desired conclusion.

(I.2) If $w \in \tilde{W}_{h}$ we will have

$$
\left|z-p_{k} / q_{k}\right| \geq \exp \left[-c\left(q_{k} q_{k+1}\right)^{h}\right],
$$

thus

$$
\left|z_{k}\right|^{-1} \leq c q_{k}^{-2} \exp \left[c\left(q_{k} q_{k+1}\right)^{h}\right]
$$

and

$$
\frac{1}{q_{k}} \log \left|z_{k}\right|^{-1} \leq c q_{k+1}^{h} q_{k}^{h-1}
$$

which is small if $\alpha$ satisfies the diophantine condition we have assumed and $k$ is large. Once again the conclusion follows from Lemma 5.20, Remark 5.18, and Theorem 5.10.

(II) Here we assume that $p / q$ is not one of the partial fractions of $\alpha$. We denote by $\left(p_{l}^{\prime} / q_{l}^{\prime}\right)_{0 \leq l \leq L}$ the partial fractions of $p / q$ and by $k$ the largest integer such that $p_{k}^{\prime} / q_{k}^{\prime}=p_{k} / q_{k}$. Clearly one has $k<L$ and $p_{L}^{\prime} / q_{L}^{\prime}=p / q$. By a classical result ([HW], Theorem 184, p. 153)

$$
|\alpha-p / q| \geq \frac{1}{2 q^{2}} .
$$

For $w \in \tilde{W}_{h}$ one has

$$
|z-p / q| \geq \exp \left[-c q^{2 h}\right]
$$

thus

$$
\left|z_{L}\right|^{-1} \leq c q^{-2} \exp \left[c q^{2 h}\right]
$$

and

$$
\frac{1}{q} \log \left|z_{L}\right|^{-1} \leq q^{-1} c q^{2 h}
$$


which is small since $h<1 / 2$. Taking into account Theorem 5.10, Lemma 5.20, and Remark 5.18 we only need to check that $B_{\text {finite }}\left(p_{k} / q_{k}\right)$ and $B_{\text {finite }}(p / q)$ are close.

Let us introduce

$$
\rho=\max _{k \leq l<L} q_{l}^{\prime-1} \log \frac{q_{l+1}^{\prime}}{q_{l}^{\prime}}(l-k+1)^{2} .
$$

By Lemma 5.20 we have

$$
\left|B_{\text {fin }}\left(p_{k} / q_{k}\right)-B_{\text {fin }}(p / q)\right| \leq c q_{k}^{-1}+c \rho,
$$

and we must show that $\rho$ is small. Let $l$ be such that

$$
\rho=q_{l}^{\prime-1} \log \frac{q_{l+1}^{\prime}}{q_{l}^{\prime}}(l-k+1)^{2} .
$$

We have

$$
\left|p / q-p_{l}^{\prime} / q_{l}^{\prime}\right| \leq \frac{1}{q_{l}^{\prime} q_{l+1}^{\prime}} .
$$

On the other hand, if $w \in \tilde{W}_{h}$ and $z=w+\alpha \in V(p / q)$, one has

$$
|z-p / q| \leq c q^{-2}
$$

from which it follows that

$$
|p / q-\alpha| \leq c(\log q)^{-1 / h}
$$

thus

$$
\left|\alpha-p_{l}^{\prime} / q_{l}^{\prime}\right| \leq \frac{1}{q_{l}^{\prime} q_{l+1}^{\prime}}+c(\log q)^{-1 / h} .
$$

By the choice (5.60) of $l$ we have

$$
q_{l+1}^{\prime}=q_{l}^{\prime} \exp \left[\frac{\rho q_{l}^{\prime}}{(l-k+1)^{2}}\right],
$$

which implies

$$
(\log q)^{-1 / h} \leq\left(\log q_{l+1}^{\prime}\right)^{-1 / h}=\left[\frac{\rho q_{l}^{\prime}}{(l-k+1)^{2}}+\log q_{l}^{\prime}\right]^{-1 / h} .
$$

When $w$ approaches zero, $q$ must be large so $k$ must be large too and if $\rho$ is not small one has by (5.59)

$$
\left[\frac{\rho q_{l}^{\prime}}{(l-k+1)^{2}}+\log q_{l}^{\prime}\right]^{-1 / h} \leq 1 / 10 q_{l}^{\prime-2}
$$

since $h<1 / 2$.

We will also have $\frac{1}{q_{l}^{\prime} q_{l+1}^{\prime}} \leq \frac{1}{10} q_{l}^{\prime-2}$, thus by (5.61)

$$
\left|\alpha-p_{l}^{\prime} / q_{l}^{\prime}\right| \leq \frac{1}{5} q_{l}^{\prime-2} .
$$

But (once again thanks to [HW], Theorem 184, p. 153) this implies that $p_{l}^{\prime} / q_{l}^{\prime}$ is one of the partial fractions of $\alpha$ and it must be $p_{k} / q_{k}$ by definition of $k$. So one has $l=k$ and

$$
\left|\alpha-p_{k} / q_{k}\right| \leq \frac{1}{q_{k} q_{k+1}^{\prime}}+c\left(\log q_{k+1}^{\prime}\right)^{-1 / h},
$$


with $q_{k+1}^{\prime}=q_{k} \exp \left(\rho q_{k}\right)$. This leads to the conclusion that $\rho$ is small if $\alpha$ verifies the diophantine condition of the second part of the theorem. If $\alpha$ is a Brjuno number and $w \in W_{H}$ the condition

$$
|z-p / q| \leq c q^{-2}
$$

implies the stronger inequality

$$
|\alpha-p / q| \leq c q^{-2 / H}
$$

thus

$$
\left|\alpha-p_{k} / q_{k}\right| \leq \frac{1}{q_{k} q_{k+1}^{\prime}}+c q_{k+1}^{-2 / H},
$$

where, once again, $q_{k+1}^{\prime}=q_{k} \exp \left(\rho q_{k}\right)$. Therefore $\rho$ must be small in this case too.

Remark 5.21. A careful examination of the previous proof leads to a slightly stronger version of the first part of Theorem 5.19 (inspired by the work of Risler Ri]). The set $B$ of Brjuno numbers $\alpha$ has an injective image into $l^{1}(\mathbb{N})$ as follows:

$$
\alpha \mapsto\left(\beta_{l-1} \log \alpha_{l}^{-1}\right)_{l \geq 0} .
$$

Let $K$ be a subset of $B$ such that its image is relatively compact in $l^{1}(\mathbb{N})$. Then the convergence

$$
\lim _{w \in W_{H}, w \rightarrow 0} \Im m \mathcal{B}(w+\alpha)=B(\alpha)
$$

is uniform w.r.t. $\alpha \in K$.

We recall that a subset $K$ of $l^{1}(\mathbb{N})$ is relatively compact if and only if

(i) $\forall n \geq 0, \exists C_{n}$ such that $\forall\left(u_{l}\right)_{l \geq 0} \in K$ one has $\left|u_{n}\right| \leq C_{n}$;

(ii) $\forall \varepsilon>0 \exists n_{0}$ such that $\forall\left(u_{l}\right)_{l \geq 0} \in K$ one has $\sum_{l>n_{0}}\left|u_{l}\right| \leq \varepsilon$.

\section{A1. Appendix 1: Real continued fractions}

In this appendix we recall some elementary facts on standard real continued fractions (we refer to [MMY], and references therein, for more general continued fractions).

We will consider the iteration of the Gauss map

$$
A:(0,1) \mapsto[0,1],
$$

defined by

$$
A(x)=\frac{1}{x}-\left[\frac{1}{x}\right] .
$$

Let

$$
G=\frac{\sqrt{5}+1}{2}, \quad g=G^{-1}=\frac{\sqrt{5}-1}{2} .
$$

To each $x \in \mathbb{R} \backslash \mathbb{Q}$ we associate a continued fraction expansion by iterating $A$ as follows. Let

$$
x_{0}=x-[x], \quad a_{0}=[x] ;
$$

then $x=a_{0}+x_{0}$. We now define inductively for all $n \geq 0$

$$
x_{n+1}=A\left(x_{n}\right), \quad a_{n+1}=\left[\frac{1}{x_{n}}\right] \geq 1,
$$


thus

$$
x_{n}^{-1}=a_{n+1}+x_{n+1} .
$$

Therefore we have

$$
x=a_{0}+x_{0}=a_{0}+\frac{1}{a_{1}+x_{1}}=\cdots=a_{0}+\frac{1}{a_{1}+\frac{1}{a_{2}+\ddots+\frac{1}{a_{n}+x_{n}}}},
$$

and we will write

$$
x=\left[a_{0}, a_{1}, \ldots, a_{n}, \ldots\right] .
$$

The $n$ th-convergent is defined by

$$
\frac{p_{n}}{q_{n}}=\left[a_{0}, a_{1}, \ldots, a_{n}\right]=a_{0}+\frac{1}{a_{1}+\frac{1}{a_{2}+\ddots \cdot+\frac{1}{a_{n}}}} .
$$

The numerators $p_{n}$ and denominators $q_{n}$ are recursively determined by

$$
p_{-1}=q_{-2}=1, \quad p_{-2}=q_{-1}=0,
$$

and for all $n \geq 0$

$$
p_{n}=a_{n} p_{n-1}+p_{n-2}, \quad q_{n}=a_{n} q_{n-1}+q_{n-2} .
$$

Moreover

$$
\begin{gathered}
x=\frac{p_{n}+p_{n-1} x_{n}}{q_{n}+q_{n-1} x_{n}}, \\
x_{n}=-\frac{q_{n} x-p_{n}}{q_{n-1} x-p_{n-1}}, \\
q_{n} p_{n-1}-p_{n} q_{n-1}=(-1)^{n} .
\end{gathered}
$$

Let

$$
\beta_{n}=\prod_{i=0}^{n} x_{i}=(-1)^{n}\left(q_{n} x-p_{n}\right) \quad \text { for } n \geq 0, \quad \text { and } \beta_{-1}=1 .
$$

From the definitions given one easily proves by induction the following proposition (see [MMY]).

Proposition A1.1. For all $x \in \mathbb{R} \backslash \mathbb{Q}$ and for all $n \geq 1$ one has

(i) $\left|q_{n} x-p_{n}\right|=\frac{1}{q_{n+1}+q_{n} x_{n+1}}$, so that $\frac{1}{2}<\beta_{n} q_{n+1}<1$;

(ii) $\beta_{n} \leq g^{n}$ and $q_{n} \geq \frac{1}{2} G^{n-1}$.

Note that from (ii) it follows that $\sum_{k=0}^{\infty} \frac{\log q_{k}}{q_{k}}$ and $\sum_{k=0}^{\infty} \frac{1}{q_{k}}$ are always convergent and their sum is uniformly bounded. With the notation of Section 2.1, equation (A1.5) can be written $x_{n}=g\left(a_{n+1}\right) x_{n+1}$, thus we have $x_{0}=g\left(a_{1}\right) g\left(a_{2}\right) \cdots g\left(a_{n}\right) x_{n}$. The following characterization of the monoid $\mathcal{M}$ defined in Section 2.1 is therefore relevant. 
Proposition A1.2. Let $g(m)=\left(\begin{array}{cc}0 & 1 \\ 1 & m\end{array}\right)$, where $m \geq 1 . \mathcal{M}$ is the free monoid, with unit, generated by the elements $g(m), m \geq 1$ : each element $g$ of $\mathcal{M}$ can be written as

$$
g=g\left(m_{1}\right) \cdots g\left(m_{r}\right), \quad r \geq 0, m_{i} \geq 1,
$$

and this decomposition is unique.

Proof. Let $\mathcal{M}^{*}$ be the monoid with unit generated by the $g(m), m \geq 1$. If $m \geq 1$, then one has $g(m) \in \mathcal{M}$ and $\mathcal{M} g(m) \subset \mathcal{M}$, thus $\mathcal{M}^{*} \subset \mathcal{M}$.

Conversely let $g \in \mathcal{M}, g \neq \mathrm{id}$. We now prove that there exists a unique integer $m \geq 1$ such that $\left(\begin{array}{ll}a^{\prime} & b^{\prime} \\ c^{\prime} & d^{\prime}\end{array}\right)=g^{\prime}=g(g(m))^{-1} \in \mathcal{M}$, which leads to the conditions $b^{\prime}=a, \quad d^{\prime}=c, a^{\prime}=b-m a, c^{\prime}=d-m c$. We consider separately three cases.

1) $a=0$, thus $b=c=1$ and $g=g(d)$, where $d \geq 1$. If there were $m \geq 1$ such that $g^{\prime}=g(g(m))^{-1} \in \mathcal{M}$, and $g^{\prime} \neq \mathrm{id}$, one should have $b^{\prime}=0$, thus $a^{\prime}=0$ and $a^{\prime} d^{\prime}-b^{\prime} c^{\prime}=0$, which is impossible.

2) $a=1$, thus $b, c \geq 1$ and $d=b c \pm 1$. We therefore have $b^{\prime}=1$ and $a^{\prime}=0$ or $a^{\prime}=1$. If $a^{\prime}=0$, then $b=m$ and $c^{\prime}=d-b c$, which is admissible if and only if $d=b c+1$. If $a^{\prime}=1$, then $b=m+1$ from which it follows that $c^{\prime}=d-m c=b c \pm 1-m c=c \pm 1$, which is admissible if and only if $d=b c-1$, and then $b, c \geq 2$.

3) $a>1$. Since $a^{\prime} \wedge b^{\prime}=1$ the relation $0 \leq a^{\prime}=b-m a \leq b^{\prime}=a$ determines uniquely $m \geq 1$ and one has $0<a^{\prime}<b^{\prime}$. But one also has $b^{\prime}=a \leq c=d^{\prime}$ and $\left|a^{\prime} d^{\prime}-b^{\prime} c^{\prime}\right|=1$, from which one easily gets $d^{\prime} \geq c^{\prime} \geq a^{\prime}$.

\section{A2. Appendix 2: Hyperfunctions}

A2.1. We follow here [H], Chapter 9 . Let $K$ be a non-empty compact subset of $\mathbb{R}$. A hyperfunction with support in $K$ is a linear functional $u$ on the space $\mathcal{O}(K)$ of functions analytic in a neighborhood of $K$ such that for all neighborhoods $V$ of $K$ there is a constant $C_{V}>0$ such that

$$
|u(\varphi)| \leq C_{V} \sup _{V}|\varphi|, \quad \forall \varphi \in \mathcal{O}(V) .
$$

We denote by $A^{\prime}(K)$ the space of hyperfunctions with support in $K$. It is a Fréchet space: a seminorm is associated to each neighborhood $V$ of $K$. One has the following proposition.

Proposition A2.1. The spaces $A^{\prime}(K)$ and $\mathcal{O}^{1}(\overline{\mathbb{C}} \backslash K)$ are canonically isomorphic. To each $u \in A^{\prime}(K)$ there corresponds $\varphi \in \mathcal{O}^{1}(\overline{\mathbb{C}} \backslash K)$ given by

$$
\varphi(z)=u\left(c_{z}\right), \forall z \in \mathbb{C} \backslash K,
$$

where $c_{z}(x)=\frac{1}{\pi} \frac{1}{x-z}$. Conversely to each $\varphi \in \mathcal{O}^{1}(\overline{\mathbb{C}} \backslash K)$ there corresponds the hyperfunction

$$
u(\psi)=\frac{i}{2 \pi} \int_{\gamma} \varphi(z) \psi(z) d z, \forall \psi \in A,
$$

where $\gamma$ is any piecewise $\mathcal{C}^{1}$ path winding around $K$ in the positive direction. We will also use the notation $u(x)=\frac{1}{2 i}[\varphi(x+i 0)-\varphi(x-i 0)]$ for short. 
A2.2. Let $\mathbb{T}^{1}=\mathbb{R} / \mathbb{Z} \subset \mathbb{C} / \mathbb{Z}$. A hyperfunction on $\mathbb{T}$ is a linear funtional $U$ on the space $\mathcal{O}\left(\mathbb{T}^{1}\right)$ of functions analytic in a complex neighborhood of $\mathbb{T}^{1}$ such that for all neighborhoods $V$ of $\mathbb{T}$ there exists $C_{V}>0$ such that

$$
|U(\Phi)| \leq C_{V} \sup _{V}|\Phi|, \quad \forall \Phi \in \mathcal{O}(V) .
$$

We will denote by $A^{\prime}\left(\mathbb{T}^{1}\right)$ the Fréchet space of hyperfunctions with support in $\mathbb{T}$. For $U \in A^{\prime}(\mathbb{T})$, let $\hat{U}(n):=U\left(e_{-n}\right)$ with $e_{n}(z)=e^{2 \pi i n z}$. The doubly infinite sequence $(\hat{U}(n))_{n \in \mathbb{Z}}$ satisfies

$$
|\hat{U}(n)|<C_{\varepsilon} e^{2 \pi|n| \varepsilon}
$$

for all $\varepsilon>0$ and for all $n \in \mathbb{Z}$ with a suitably chosen $C_{\varepsilon}>0$. Conversely any such sequence is the Fourier expansion of a unique hyperfunction with support in $\mathbb{T}$.

Let $\mathcal{O}_{\Sigma}$ denote the complex vector space of holomorphic functions $\Phi: \mathbb{C} \backslash \mathbb{R} \rightarrow \mathbb{C}$, 1-periodic, bounded at $\pm i \infty$ and such that $\Phi( \pm i \infty):=\lim _{\mathfrak{I} m z \rightarrow \pm \infty} \Phi(z)$ exist and verify $\Phi(+i \infty)=-\Phi(-i \infty)$.

Proposition A2.2. The spaces $A^{\prime}\left(\mathbb{T}^{1}\right)$ and $\mathcal{O}_{\Sigma}$ are canonically isomorphic. To each $U \in A^{\prime}\left(\mathbb{T}^{1}\right)$ there corresponds $\Phi \in \mathcal{O}_{\Sigma}$ given by

$$
\Phi(z)=U\left(C_{z}\right), \forall z \in \mathbb{C} \backslash K,
$$

where $C_{z}(x)=\operatorname{cotg} \pi(x-z)$. Conversely to each $\Phi \in \mathcal{O}_{\Sigma}$ there corresponds the hyperfunction

$$
U(\Psi)=\frac{i}{2} \int_{\Gamma} \Phi(z) \Psi(z) d z, \forall \Psi \in A\left(\mathbb{T}^{1}\right),
$$

where $\Gamma$ is any piecewise $\mathcal{C}^{1}$ path winding around a closed interval $I \subset \mathbb{R}$ of length 1 in the positive direction. We will also use the notation

$$
U(x)=\frac{1}{2 i}[\Phi(x+i 0)-\Phi(x-i 0)]
$$

for short.

The nice fact is that the following diagram commutes:

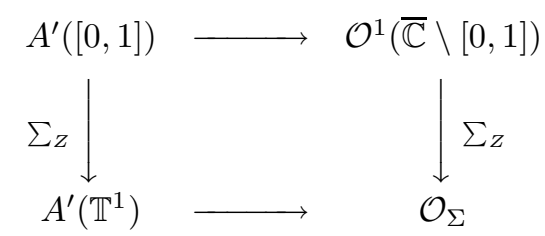

Here, the horizontal lines are the above-mentioned isomorphisms and $\sum_{Z}$ is defined in 2.2 .2 .

\section{A3. Appendix 3: Some Properties of the Dilogarithm}

A3.1. The classical dilogarithmic series (see [Le, [O] for more information) is defined by

$$
\operatorname{Li}_{2}(z)=\sum_{n=1}^{+\infty} \frac{z^{n}}{n^{2}}
$$


and it is convergent for $|z| \leq 1$. Since $-\log (1-z)=\sum_{n=1}^{+\infty} \frac{z^{n}}{n}$, dividing by $z$ and integrating one obtains the analytic continuation of the dilogarithm to $\mathbb{C} \backslash[1,+\infty)$ by means of the integral formula

$$
\mathrm{Li}_{2}(z)=-\int_{0}^{z} \frac{\log (1-t)}{t} d t=\int_{0}^{z}\left(\int_{0}^{t} \frac{d \zeta}{1-\zeta}\right) \frac{d t}{t}
$$

which we will use as a definition of the dilogarithm. Note that $[1,+\infty)$ is a branch cut.

Since

$$
\operatorname{Li}_{2}(z)=z \int_{0}^{1} \frac{\log t}{t z-1} d t
$$

one obviously has that

$$
\operatorname{Li}_{2}\left(\frac{1}{z}\right)=-\int_{0}^{1} \frac{\log t}{z-t} d t
$$

which shows that $\operatorname{Li}_{2}\left(\frac{1}{z}\right)$ is the Cauchy-Hilbert transform of the real function

$$
\varphi_{0}(t)=\left\{\begin{array}{cc}
-\log t & \text { if } t \in[0,1] \\
0 & \text { elsewhere }
\end{array}\right.
$$

Note also that

$$
\mathfrak{I} m \mathrm{Li}_{2}(t \pm i 0)= \pm \pi \log t
$$

where $t \in[1,+\infty)$. Moreover

$$
\left|\operatorname{Li}_{2}(z)\right|=\mathcal{O}\left(\log ^{2}|z|\right) \quad \text { as }|z| \rightarrow+\infty .
$$

A3.2. Euler's functional equations.

$$
\begin{aligned}
\operatorname{Li}_{2}(z)+\mathrm{Li}_{2}\left(\frac{1}{z}\right) & =-\frac{1}{2}(\log (-z))^{2}-\frac{\pi^{2}}{6}, \\
\mathrm{Li}_{2}(z)+\mathrm{Li}_{2}(1-z) & =-\log z \log (1-z)+\frac{\pi^{2}}{6},
\end{aligned}
$$

where $z$ varies in $\mathbb{C} \backslash[0,+\infty]$ and $\mathbb{C} \backslash((-\infty, 0] \cup[1,+\infty))$ respectively.

A3.3. Special values.

$$
\begin{aligned}
\operatorname{Li}_{2}(1) & =\frac{\pi^{2}}{6}, \operatorname{Li}_{2}(-1)=-\frac{\pi^{2}}{12} \\
2 \mathrm{Li}_{2}\left(\frac{1}{2}\right) & =\frac{\pi^{2}}{6}-(\log 2)^{2}, \operatorname{Li}_{2}(2 \pm i 0)=\frac{\pi^{2}}{4} \pm \pi i \log 2 .
\end{aligned}
$$

\section{A4. Appendix 4: Even Bruuno functions}

In MMY we also considered an even version of the Brjuno function and we proved that this differs from the one considered here by a $1 / 2$-Hölder continuous function. In this appendix we explicitly state the relation among the two associated complex Brjuno functions. 
A4.1. Let $\sigma$ denote the matrix $\left(\begin{array}{cc}-1 & 1 \\ 0 & 1\end{array}\right)$ which corresponds to $x \mapsto 1-x$.

At the real level, replacing periodic even functions with functions on $[0,1 / 2]$ and null outside this interval, the operator $T_{\text {even }}$ acting on $L^{2}([0,1 / 2])$ (for example) can be written explicitly as

$$
T_{\text {even }} f(x)=\sum_{m \geq 2} x f\left(\frac{1}{x}-m\right)+\sum_{m \geq 3} x f\left(m-\frac{1}{x}\right) .
$$

At the complex level (i.e. after the identification of $\mathcal{A}^{\prime}([0,1 / 2])$ to $\left.\mathcal{O}^{1}(\overline{\mathbb{C}} \backslash[0,1 / 2])\right)$ one gets

$$
T_{\text {even }} \varphi=\sum_{m \geq 2} L_{g(m)} \varphi+\sum_{m \geq 2} L_{g^{\prime}(m)} \varphi
$$

where $g^{\prime}(m)=\left(\begin{array}{cc}0 & 1 \\ -1 & m+1\end{array}\right)=g(m) \sigma$.

A4.2. We want to consider

$$
\left(1-T_{\text {even }}\right)^{-1}: \mathcal{O}^{1}(\overline{\mathbb{C}} \backslash[0,1 / 2]) \rightarrow \mathcal{O}^{1}(\overline{\mathbb{C}} \backslash[0,1 / 2])
$$

and then one will have to make the resulting function even and periodic, thus one will take

$$
\sum_{Z}\left(1+L_{\sigma}\right)\left(1-T_{\text {even }}\right)^{-1}: \mathcal{O}^{1}(\overline{\mathbb{C}} \backslash[0,1 / 2]) \rightarrow \mathcal{O}_{\text {even }}(\mathbb{H} / \mathbb{Z}) .
$$

Note that $Z \sqcup Z \sigma=Z \sqcup \sigma Z$.

When we expand $\left(1+L_{\sigma}\right)\left(1-T_{\text {even }}\right)^{-1}$ we obtain a sum $\sum L_{g}$ where the matrices $g$ have the form

$$
g=\varepsilon_{0} g\left(i_{1}\right) \varepsilon_{1} g\left(i_{2}\right) \cdots g\left(i_{r}\right) \varepsilon_{r}
$$

with $r \geq 0, i_{k} \geq 2$ and $\varepsilon_{k} \in\{1, \sigma\}$.

Note that $\sigma g(i)=g(1) g(i-1)$ for all $i \geq 2$, thus all matrices of the form $\varepsilon_{0} g\left(i_{1}\right) \varepsilon_{1} \cdots g\left(i_{r}\right)$ belong to the monoid $\mathcal{M}$.

A4.3. Let $r \geq 0$,

$$
\mathcal{M}^{(r)}=\left\{g\left(j_{1}\right) \cdots g\left(j_{r}\right), j_{k} \geq 1\right\}
$$

and let $\widehat{\mathcal{M}}^{(r)}$ denote the part of $\mathcal{M}^{(r)}$ made of matrices which can be written as a product $\varepsilon_{0} g\left(i_{1}\right) \varepsilon_{1} \cdots g\left(i_{s}\right)$. We have the following

Lemma A4.1. Each matrix $g \in \widehat{\mathcal{M}}^{(r)}$ can be written in a unique way as a product of matrices $\varepsilon_{0} g\left(i_{1}\right) \varepsilon_{1} \cdots g\left(i_{s}\right)$. Moreover one has $\mathcal{M}^{(0)}=\widehat{\mathcal{M}}^{(0)}=\{1\}$ and for all $r>0$

$$
\mathcal{M}^{(r)} \backslash \widehat{\mathcal{M}}^{(r)}=\widehat{\mathcal{M}}^{(r-1)} g(1) .
$$

Proof. Uniqueness is evident (just consider the first place at which the product $\varepsilon_{0} g\left(i_{1}\right) \varepsilon_{1} \cdots g\left(i_{s}\right)$ differs from $\left.\varepsilon_{0}^{\prime} g\left(i_{1}^{\prime}\right) \varepsilon_{1}^{\prime} \cdots g\left(i_{s^{\prime}}^{\prime}\right)\right)$. The second assertion follows easily from the remark that $\widehat{\mathcal{M}}^{(r)}$ is indeed made of matrices $g=g\left(j_{1}\right) \cdots g\left(j_{r}\right)$ which end with an even number of $g(1)$ 's. 
Let $\widehat{\mathcal{M}}=\bigsqcup_{r \geq 0} \widehat{\mathcal{M}}^{(r)}$ and $\sum_{\widehat{\mathcal{M}}}=\sum_{g \in \widehat{\mathcal{M}}} L_{g}$. One clearly has

$$
\left(1+L_{\sigma}\right) \circ\left(1-T_{\text {even }}\right)^{-1}=\left(\sum_{\widehat{\mathcal{M}}}\right) \circ\left(1+L_{\sigma}\right)
$$

and by the previous lemma

$$
\begin{aligned}
\sum_{\mathcal{M}}=\sum_{g \in \mathcal{M}} L_{g} & =\sum_{r \geq 0} \sum_{g \in \mathcal{M}^{(r)}} L_{g}=\sum_{r \geq 0}\left(\sum_{g \in \widehat{\mathcal{M}}^{(r)}} L_{g}+\sum_{g \in \mathcal{M}^{(r)} \backslash \widehat{\mathcal{M}}^{(r)}} L_{g}\right) \\
& =\sum_{r \geq 0} \sum_{g \in \widehat{\mathcal{M}}^{(r)}} L_{g}+\sum_{r \geq 1} \sum_{g \in \widehat{\mathcal{M}}^{(r-1)} g(1)} L_{g}=\left(\sum_{\widehat{\mathcal{M}}}\right) \circ\left(1+L_{g(1)}\right) .
\end{aligned}
$$

We are therefore led to conclude that

$$
\left(1+L_{\sigma}\right) \circ\left(1-T_{\text {even }}\right)^{-1}=\left(\sum_{\mathcal{M}}\right) \circ\left(1+L_{g(1)}\right)^{-1} \circ\left(1+L_{\sigma}\right) .
$$

A4.4. It is not hard to check, as we did for the monoid $\mathcal{M}$ in Proposition A1.2, that a matrix $g$ belongs to the monoid $\widehat{\mathcal{M}}$ if and only if $d \geq 2 b>0, c \geq 2 a \geq 0$, $d \geq G c$, where $G=\frac{\sqrt{5}+1}{2}$. Moreover the decomposition $g=\varepsilon_{0} g\left(i_{1}\right) \varepsilon_{1} \cdots g\left(i_{r}\right)$ is unique.

\section{A5. Appendix 5: The Real Bruuno function as a Cocycle}

In this Appendix we show how to interpret the real Brjuno function as a cocycle under the action of $\operatorname{PGL}(2, \mathbb{Z})$ on $\mathbb{R} \backslash \mathbb{Q}$. To this purpose we first recall some basic definitions taken from the cohomology of groups. We refer to [Ja] and Se] for more information and the proofs.

A5.1. Group cohomology. Let $G$ be a group and $M$ an abelian group with a left $G$-action, i.e. a structure of a left $\mathbb{Z}^{G}$-module. Recall that for $n \geq 0$, one defines

(i) the abelian group of $n$-cochains $C^{n}(G, M)$, whose elements are applications from $G^{n}$ to $M$.

(ii) the coboundary operator $d^{n}: C^{n}(G, M) \rightarrow C^{n+1}(G, M)$ :

$$
\begin{aligned}
\left(d^{n} f\right)\left(g_{0}, \ldots, g_{n}\right) & =g_{0} f\left(g_{1}, \ldots, g_{n}\right)+\sum_{i=0}^{n-1}(-1)^{i+1} f\left(g_{0}, \ldots, g_{i} g_{i+1}, \ldots, g_{n}\right) \\
& +(-1)^{n+1} f\left(g_{0}, \ldots, g_{n-1}\right)
\end{aligned}
$$

(iii) the abelian subgroups of $n$-cocycles $Z^{n}(G, M)=\operatorname{Ker} d^{n}$ and of $n$-coboundaries, $B^{n}(G, M)=\operatorname{Im} d^{n-1}$;

(iv) the $n$-th cohomology group $H^{n}=Z^{n}(G, M) / B^{n}(G, M)$.

Identifying $C^{0}(G, M)$ with $M$, one has $H^{0}(G, M)=Z^{0}(G, M)=\{m \in M ; g m$ $=m$ for all $g \in G\}$ with $M$. An application $c: G \rightarrow M$ is a 1 -cocycle iff $c\left(g_{0} g_{1}\right)=$ $g_{0} c\left(g_{1}\right)+c\left(g_{0}\right)$, and a 1 -coboundary iff $c(g)=g \cdot m-m$ for some $m$ and all $g \in G$. 
A5.2. Automorphic factors, cocycles and coboundaries. Let $G$ be a group acting on the left on a set $X$. Let $A$ be an abelian ring, $A^{*}$ the multiplicative group of invertible elements of $A$, and $M$ a $A$-module. A function $\chi: G \times X \rightarrow A$ is an automorphic factor if the application $G \times M^{X} \longrightarrow M^{X}$ given by

$$
(g, \varphi) \longmapsto g \cdot \varphi: g \cdot \varphi(x)=\chi\left(g^{-1}, x\right) \varphi\left(g^{-1} \cdot x\right) \forall x \in X,
$$

defines a left action of $G$ on $M^{X}$ : one must have

$$
\chi\left(g_{0} g_{1}, x\right)=\chi\left(g_{0}, g_{1} x\right) \chi\left(g_{1}, x\right) .
$$

One has therefore given to $M^{X}$ the structure of a $\mathbb{Z}^{[G]}$-module. The coboundary of an element $\varphi \in M^{X}$ is given by $d^{0} \varphi(g)=g \cdot \varphi-\varphi$, i.e.

$$
d^{0} \varphi(g)(x)=\chi\left(g^{-1}, x\right) \varphi\left(g^{-1} \cdot x\right)-\varphi(x) \forall x \in X .
$$

A 1-cocycle is an application $c: G \rightarrow M^{X}$ verifying $g_{0} \cdot c\left(g_{1}\right)-c\left(g_{0} g_{1}\right)+c\left(g_{0}\right)=0$, i.e., letting $\check{c}(g)=c\left(g^{-1}\right)$ :

$$
\check{c}\left(g_{0} g_{1}\right)=c\left(g_{1}^{-1} g_{0}^{-1}\right)=c\left(g_{1}^{-1}\right)+g_{1}^{-1} c\left(g_{0}^{-1}\right)=\check{c}\left(g_{1}\right)+g_{1}^{-1} \check{c}\left(g_{0}\right),
$$

or, equivalently, $\check{c}\left(g_{0} g_{1}, x\right)=\chi\left(g_{1}, x\right) \check{c}\left(g_{0}, g_{1} \cdot x\right)+\check{c}\left(g_{1}, x\right) \forall x \in X$.

A5.3. Action of $\operatorname{PGL}(2, \mathbb{Z})$ on $\mathbb{R} \backslash \mathbb{Q}$. Let us consider $G=\operatorname{PGL}(2, \mathbb{Z})$ and $X=\mathbb{R} \backslash \mathbb{Q}$, the action being given by the homographies. The transformations $T(x)=x+1$ and $S(x)=x^{-1}$ generate $\operatorname{PGL}(2, \mathbb{Z})$. One has the following more precise result:

Proposition A5.1. Let $g \in \operatorname{PGL}(2, \mathbb{Z})$ and let $x_{0} \in \mathbb{R} \backslash \mathbb{Q}$. There exist $r \geq 0$ and elements $g_{1}, \ldots, g_{r} \in\left\{S, T, T^{-1}\right\}$ such that

(i) $g=g_{r} \cdots g_{1}$;

(ii) let $x_{i}=g_{i} x_{i-1}$ for $1 \leq i \leq r$, then $x_{i-1}>0$ if $g_{i}=S$.

Moreover one can require that $g_{i} g_{i-1} \neq 1$ for $0<i \leq r$, and in this case $r, g_{1}, \ldots, g_{r}$ are uniquely determined.

Proof. First we prove the existence. Let $U(x)=-x$. We consider five cases:

1. If $g=T^{ \pm 1}$, any $x_{0}$, one takes $r=1, g_{1}=T^{ \pm 1}$.

2. If $g=U$ and $x_{0} \in(0,1)$, then $r=6$ and $g_{1}=S, x_{1}=x_{0}^{-1} ; g_{2}=T^{-1}$, $x_{2}=\frac{1-x_{0}}{x_{0}} ; g_{3}=S, x_{3}=\frac{x_{0}}{1-x_{0}} ; g_{4}=T, x_{4}=\frac{1}{1-x_{0}} ; g_{5}=S, x_{5}=1-x_{0} ;$ $g_{6}=T^{-1}, x_{6}=-x_{0}$.

3. If $g=U$ and $x_{0} \in(n, n+1), n \in \mathbb{Z}$, one is led to consider the previous case by using $U=T^{-n} U T^{-n}$.

4. If $g=S$ it is immediate if $x_{0}>0$, and if $x_{0}<0$ one is led to consider case 3 by using the relation $S=U S U$.

5. One has the cases $g=S$ and $g=T^{ \pm 1}$ for all $x_{0}$. Since $S$ and $T$ generate $\mathrm{PGL}(2, \mathbb{Z})$ this implies the existence in all possible cases.

We can now prove uniqueness. It is sufficient to show that if $r>0$ and $g_{1}, \ldots, g_{r} \in\left\{S, T, T^{-1}\right\}, x_{0} \in \mathbb{R} \backslash \mathbb{Q}$ verify

$$
g_{r} \cdots g_{1}=1 \text { and } x_{i-1}>0 \text { if } g_{i}=S(1 \leq i \leq r),
$$

then there exists $1<i \leq r$ such that $g_{i} g_{i-1}=1$. We prove this by contradiction: let $r$ be minimal, $r>0$.

If $x_{0}<0$ one must have $r \geq 2, g_{1}=T$ and $g_{r}=T^{-1}$, thus $r \geq 3$ and $g_{r-1} \cdots g_{2}=1$, which contradicts the minimality of $r$. 
One is led to assume $x_{0}>0$. Clearly one must have $x_{i}>0$ for all $i \in[0, r]$. Let $i_{1}<i_{2}<\cdots<i_{k}$ denote the indices $i$ such that $g_{i}=S$. The integer $k>0$ is even because of the determinant sign. Let us assume that $x_{i_{1}-1}>1$. Then $x_{i_{1}} \in(0,1)$, thus $x_{i_{2}-1}=x_{i_{1}}+\left(i_{2}-i_{1}-1\right)>1$. Therefore $x_{i_{l}-1}>1$ for all $1 \leq l \leq k$. But then $\prod_{1}^{r} \frac{d g_{i}}{d x_{i-1}}\left(x_{i-1}\right)<1$, in contradiction with the assumption $g_{r} \cdots g_{1}=1$. If there exists $l$ such that $x_{i_{l}-1}<1$, then one permutes circularly all $g_{i}$ and $x_{i}$ $(\bmod r)$ until one is back to the case previously considered. Finally if $x_{i_{l}-1}<1$ for all $1 \leq l \leq k$, then $\prod_{1}^{r} \frac{d g_{i}}{d x_{i-1}}\left(x_{i-1}\right)>1$, which is again in contradiction with the assumption $g_{r} \cdots g_{1}=1$.

Corollary A5.2. Let $A$ be an abelian ring, and let the maps $T$ and $S$ be such that $t: \mathbb{R} \backslash \mathbb{Q} \rightarrow A^{*}, s:(0,1) \cap(\mathbb{R} \backslash \mathbb{Q}) \rightarrow A^{*}$. There exists a unique automorphic factor $\chi$ such that

$$
\begin{aligned}
& \chi(T, x)=t(x) \text { for all } x \in \mathbb{R} \backslash \mathbb{Q}=X, \\
& \chi(S, x)=s(x) \text { for all } x \in X \cap(0,1) .
\end{aligned}
$$

Proof. Let $s(x)=\left(s\left(x^{-1}\right)\right)^{-1}$ for all $x \in X \cap(1,+\infty)$. The map $s$ is therefore defined on $X \cap(0,+\infty)$ and one must have

$$
\chi(S, x)=s(x) \text { for all } x \in X, x>0 .
$$

a) The uniqueness of $\chi$ follows from the existence in the previous proposition: if $g \in \operatorname{PGL}(2, \mathbb{Z})$ and $x_{0} \in X$ one must have

$$
\chi\left(g, x_{0}\right)=\prod_{i=1}^{r} \chi\left(g_{i}, x_{i-1}\right)
$$

where $g_{1}, \ldots, g_{r}$ and $x_{1}, \ldots, x_{r}$ are defined in the proposition and

$$
\begin{aligned}
\chi(T, x) & =t(x) \text { for all } x \in X, \\
\chi\left(T^{-1}, x\right) & =(t(x-1))^{-1} \text { for all } x \in X, \\
\chi(S, x) & =s(x) \text { for all } x \in X, x>0 .
\end{aligned}
$$

b) The existence of $\chi$ follows from the uniqueness in the previous proposition: here we use (1) with $r$ minimal (i.e. $g_{i} g_{i-1} \neq 1$ for all $1<i \leq r$ ) to define $\chi$ and one must check that

$$
\chi\left(g^{\prime} g, x_{0}\right)=\chi\left(g^{\prime}, g x_{0}\right) \chi\left(g, x_{0}\right) .
$$

Let $y_{0}=x_{r}=g x_{0}, \quad g^{\prime}=g_{s}^{\prime} \cdots g_{1}^{\prime}$, following the previous proposition, and let

$$
g^{\prime \prime}=g^{\prime} g, \quad g_{i}^{\prime \prime}=\left\{\begin{array}{cl}
g_{i} & \text { if } 1 \leq i \leq r, \\
g_{i-s}^{\prime} & \text { if } r<i \leq r+s,
\end{array} \quad x_{i}=y_{i-r} .\right.
$$

Then $g^{\prime \prime}=g_{r+s}^{\prime \prime} \cdots g_{1}^{\prime \prime}$ satisfies the conclusions of the proposition. The decomposition may not be minimal (since one may have $g_{1}^{\prime} g_{r}=1$ ) but one can obtain a minimal decomposition by deleting $g_{1}^{\prime} g_{r}$ if $g_{1}^{\prime} g_{r}=1$, then (if $g_{1}^{\prime} g_{r}=1$ ) by deleting $g_{2}^{\prime} g_{r-1}$ if $g_{2}^{\prime} g_{r-1}=1$, and so on. Given the definition (1) of $\chi$ the automorphic property is now verified if

$$
\chi\left(g, g^{-1} x\right) \chi\left(g^{-1}, x\right)=1
$$

when $g=T, T^{-1}$ or $S$ and $x>0$ if $g=S$, which is immediate to check. 
Corollary A5.3. Let $A$ be an abelian ring, $\chi$ an automorphic factor, $M$ an $A-$ module $M^{X}$ with the structure of the $\mathbb{Z}^{[G]}$-module defined by $\chi$. Let

$$
\begin{aligned}
& \check{c}_{T}: X \rightarrow M, \\
& \check{c}_{S}: X \cap(0,1) \rightarrow M
\end{aligned}
$$

denote two maps. There exists a unique cocycle $\check{c}: G \times M \rightarrow M$ such that

$$
\begin{aligned}
& \check{c}(T ; x)=\check{c}_{T}(x) \text { for all } x \in X, \\
& \check{c}(S ; x)=\check{c}_{S}(x) \text { for all } x \in X \cap(0,1) .
\end{aligned}
$$

Proof. One must have

$$
\check{c}\left(T^{-1} ; x\right)=-\chi\left(T^{-1}, x\right) \check{c}_{T}(x-1) \text { for all } x \in X
$$

and

$$
\check{c}(S ; x)=-\chi(S, x) \check{c}_{S}\left(x^{-1}\right) \text { for all } x \in X, x>1 .
$$

Moreover, if $g=g_{r} \cdots g_{1}$ and $x_{0}$ are given as in the proposition, then

$$
\check{c}\left(g ; x_{0}\right)=\sum_{i=1}^{r}\left(\check{c}\left(g_{i}, x_{i-1}\right) \chi\left(g_{i-1} \cdots g_{1}, x_{0}\right)\right)
$$

from which the uniqueness follows. The proof of existence is the same as the one given for Corollary A5.2.

A5.4. The real Brjuno function as a cocycle. Let $A=\mathbb{R}, t(x)=1$ and $s(x)=\varepsilon x^{\nu}$ with $\varepsilon \in\{-1,+1\}, \nu \in \mathbb{R}$ and apply Corollary A5.2. Then

$$
\begin{aligned}
\chi\left(T^{n}, x\right) & =1, \text { for all } n \in \mathbb{Z}, x \in X, \\
\chi(S, x) & =\varepsilon x^{\nu}, \text { for all } x \in X, x>0 .
\end{aligned}
$$

If $x_{0} \in(0,1)$, one has seen that $U=T^{-1} S T S T^{-1} S$, thus

$$
\chi\left(U, x_{0}\right)=\varepsilon x_{0}^{\nu} \varepsilon\left(\frac{1-x_{0}}{x_{0}}\right)^{\nu} \varepsilon\left(\frac{1}{1-x_{0}}\right)^{\nu}=\varepsilon .
$$

From $U=T^{n} U T^{n}$ it follows that $\chi(U, x)=\varepsilon$ for all $x \in X$, and from $S=U S U$ it follows that $\chi(S, x)=\varepsilon \varepsilon|x|^{\nu} \varepsilon$ for $x<0$, or $\chi(S, x)=\varepsilon|x|^{\nu}$ for all $x \in X$. One concludes that one must have

$$
\chi(g, x)=\left\{\begin{array}{cc}
|c x+d|^{\nu} & \text { if } \varepsilon=+1, \\
\operatorname{det}(g)|c x+d|^{\nu} & \text { if } \varepsilon=-1
\end{array}\right.
$$

for all $g=\left(\begin{array}{ll}a & b \\ c & d\end{array}\right) \in \operatorname{PGL}(2, \mathbb{Z})$.

Consider now the functional equations

$$
\begin{aligned}
& B_{f}(x)=x B_{f}(1 / x)+f(x), \quad x \in(0,1) \cap \mathbb{R} \backslash \mathbb{Q}, \\
& B_{f}(x)=B_{f}(x+1), \quad x \in \mathbb{R} \backslash \mathbb{Q},
\end{aligned}
$$

where $f:(0,1) \cap \mathbb{R} \backslash \mathbb{Q} \rightarrow \mathbb{C}$ is given. Now we look for $B_{f}: \mathbb{R} \backslash \mathbb{Q} \rightarrow \mathbb{C}$, and we easily see that the relevant automorphic factor is the case $\varepsilon=+1, \nu=+1$ above (other values of $\nu$ have also been considered in [MMY]). By Corollary A5.3, there exists exactly one $1-\operatorname{cocycle} c_{f}$ such that

$$
\begin{aligned}
& C_{f}(T, x)=0 \quad \forall x \in \mathbb{R} \backslash \mathbb{Q}, \\
& C_{f}(S, x)=f(x) \quad \forall x \in \mathbb{R} \backslash \mathbb{Q} \cap(0,1) .
\end{aligned}
$$


The 1-cocycle is a 1-coboundary if and only if the functional equations have a solution $B_{f}$, in which case we have $c_{f}=-d^{0}\left(B_{f}\right)$. These considerations also apply and may become fruitful in case we restrict $\mathbb{C}^{\mathbb{R} \backslash \mathbb{Q}}$ to one of its $\mathbb{C}^{[G]}$-submodules, for instance measurable functions.

\section{REFERENCES}

[BG1] A. Berretti and G. Gentile, Scaling properties for the radius of convergence of the Lindstedt series: the standard map, J. Math. Pures Appl. (9) 78 (1999), no. 2, 159-176. MR 2000c:37054

[BG2] A. Berretti and G. Gentile, Bryuno function and the standard map, University of Roma (Italy), Preprint (1998).

[BPV] N. Buric, I. Percival and F. Vivaldi, Critical function and modular smoothing, Nonlinearity 3 (1990), 21-37. MR 90m:58062

[Br1] A. D. Brjuno, Analytical form of differential equations, Trans. Moscow Math. Soc. 25 (1971), 131-288. MR 50:9476

[Br2] A. D. Brjuno, Analytical form of differential equations, Trans. Moscow Math. Soc. 26 (1972), 199-239. MR 50:9476

[Da1] A. M. Davie, The critical function for the semistandard map, Nonlinearity 7 (1994), 219-229. MR 95f:58067

[Da2] A. M. Davie, Renormalisation for analytic area-preserving maps, University of Edinburgh preprint (1995).

[Du] P. L. Duren, Theory of $H^{p}$ Spaces, Academic Press, New York, 1970. MR 42:3552

[Ga] J. B. Garnett, Bounded Analytic Functions, Academic Press, New York, 1981. MR 83g:30037

[GCRF] J. Garcia-Cuerva and J. L. Rubio de Francia, Weighted Norm Inequalities and Related Topics, vol. 116, North Holland Mathematical Studies, Amsterdam, 1985. MR 87d:42023

[HW] G. H. Hardy and E. M. Wright, An Introduction to the Theory of Numbers (Fifth Edition), Oxford Science Publications, 1979. MR 81i:10002

[H] L. Hörmander, The Analysis of Linear Partial Differential Operators I, vol. 256, Grundlehren der mathematischen Wissenschaften, Springer-Verlag, Berlin, Heidelberg, New York, Tokyo, 1983. MR 85g:35002a

[Ja] N. Jacobson, Basic Algebra I and II, Freeman, San Francisco, 1980. MR [50:9457] MR 81g:00001

[KH] A. Katok and B. Hasselblatt, Introduction to the Modern Theory of Dynamical Systems, vol. 54, Encyclopedia of Mathematics and its Applications, Cambridge University Press, 1995. MR 96c:58055

[Le] L. Lewin, Polylogarithms and Associated Functions, Elsevier North-Holland, New York, 1981. MR 83b:33019

[Ma] S. Marmi, Critical functions for complex analytic maps, J. Phys. A: Math. Gen. 23 (1990), 3447-3474. MR 92b:58199

[MMY] S. Marmi, P. Moussa and J.-C. Yoccoz, The Brjuno functions and their regularity properties, Commun. Math. Phys. 186 (1997), 265-293. MR 98e:58137

[O] J. Oesterlé, Polylogarithmes, Séminaire Bourbaki n. 762, Astérisque 216 (1993), 49-67. MR 94m:11135

[Ri] E. Risler, Linéarisation des perturbations holomorphes des rotations et applications, Mémoires Soc. Math. France 77 (1999). CMP 2000:17

[S] C. L. Siegel, Iteration of analytic functions, Annals of Mathematics 43 (1942), 807-812. MR 4:76c

[Se] J.-P. Serre, Cohomologie Galoisienne, vol. 5, Lecture Notes in Mathematics, SpringerVerlag, Berlin, 1973. MR 53:8030

[St] E. M. Stein, Singular Integrals and Differentiability Properties of Functions, Princeton University Press, 1970. MR 44:7280

[Yo1] J.-C. Yoccoz, Théorème de Siegel, nombres de Bruno et polynômes quadratiques, Astérisque 231 (1995), 3-88. MR 96m:58214 
[Yo2] J.-C. Yoccoz, An introduction to small divisors problems, From Number Theory to Physics (M. Waldschmidt, P. Moussa, J.-M. Luck and C. Itzykson, eds.), Springer-Verlag, 1992, pp. 659-679. MR 94h:58148

[Yo3] J.-C. Yoccoz, Analytic linearisation of analytic circle diffeomorphisms, in preparation.

Dipartimento di Matematica e Informatica, Università di Udine, Via delle Scienze 206, Loc. RizZi, I-33100 Udine, Italy

E-mail address: marmi@dimi.uniud.it

Current address: Scuola Normale Superiore, Classe di Scienze, Piazza dei Cavalieri 7, I-56126 Pisa, Italy

E-mail address: marmi@sns.it

Service de Physique Théorique, CeA/Saclay, 91191 Gif-Sur-Yvette, France

E-mail address: moussa@spht.saclay.cea.fr

Collège de France, 3 Rue d’Ulm, F-75005 Paris, France, and Université de Paris-Sud, Mathématiques, Batiment 425, F-91405 Orsay, France

E-mail address: jean-c.yoccoz@college-de-france.fr 